\title{
The Role of Gata3 in Blood Stem Cell
}

\author{
Emergence
}

Nada M. O. Zaidan

Murray Edwards College

University of Cambridge

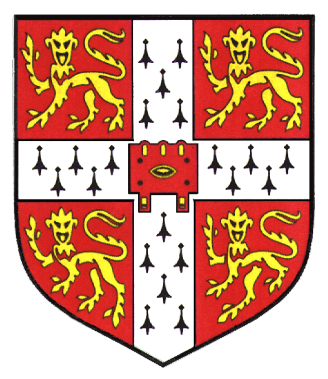

This dissertation is submitted for the degree of

Doctor of Philosophy

September 2017 


\section{Declaration}

This dissertation is the result of my own work and includes nothing, which is the outcome of work done in collaboration except as declared in the Preface and specified in the text. One of the experiments described in this thesis were done in collaboration; the RNA-Sequencing and analysis. Specific author contributions are described in the Methods.

I further state that no substantial part of my dissertation has already been submitted, or, is being concurrently submitted for any such degree, diploma or other qualification at the University of Cambridge or any other University of similar institution except as declared in the Preface and specified in the text.

It does not exceed the prescribed word limit for the relevant Degree Committee. Clinical Medicine and Clinical Veterinary Medicine instruction are as following: For the $\mathrm{PhD}$ degree, not to exceed 60,000 words. These limits exclude figures, photographs, tables, appendices and bibliography. 


\section{Acknowledgement}

First and foremost, I would like to thank my supervisor, Dr. Katrin Ottersbach. Her guidance, patience, kindness, motivation and continuous support throughout the years have made her the best mentor a student could hope for. I appreciate all of her contributions of time and ideas towards making my $\mathrm{PhD}$ experience productive and stimulating. I am also thankful for the excellent example she has provided as a successful woman and mentor.

I would like to thank all the members of Ottersbach group, previous and current. In particular, I would like to thank Dr. Simon Fitch for his guidance, insightful remarks and help during the first two years of my PhD. To the rest of the group, especially Dr.Camille Malouf, I am grateful for the helpful discussions, technical support, yummy snacks and great work atmosphere. I would also like to thank all the wonderful people that I've had the chance to work with in CIMR and SCRM, in both core facilities and labs. Their friendship and helpfulness has greatly enriched my PhD experience.

In CIMR, I would like to especially thank Prof. Berthold Gottgens and his group, in particular Dr. Nicola Wilson and Lila Diamanti. Their help and supervision with the RNA Seq experiment and analysis was invaluable.

In SCRM, I would like to thank Prof. Alexander Medvisnky and his group, in particular Dr. Celine Souilhol and Dr. Stanislav Rybtsov, for showing me the tricks of the co-aggregates and cell cycle experiments, and for their help and support in general.

Also, I would like to thank the wonderful flow cytometry team at SCRM, both Fiona Rossi and Dr. Claire Cryer, for starting as early as 8am or finishing as late as $8 \mathrm{pm}$ in order to capture the right stage of my cells. For that, I am especially grateful. 
I'm also grateful to Dr. Mariella de Bruijn for providing us with the protocol of OP9 co-culture.

I would like to sincerely thank my family and friends, for they were my support system that kept me sane during my PhD years. I am eternally grateful and thankful to them: to my dad for his encouragement and strength, my mom for her unwavering faith and support, Amal for her infectious optimism, Shatha for her motivation, Reda for his kindness, and my wonderful niece and nephews for always cheering me up and making me smile. I'm deeply grateful to my fluffy companion, Tita, for her unconditional love and companionship throughout the years.

I would also like to thank the rest of my family and friends - you are too many to list here but you know who you are! However, I do want to mention few by name... I am grateful to two of my uncles, Ibrahim and Mohammed, whom both passed on during my $\mathrm{PhD}$, for their encouragement and support when I first started graduate school. I'm also grateful to Esmina von Habsburg, Joan Lee, Carmen Ferraro, Raja'a Alahmadi and Alanoud Bin Khuthaila. Thank you for your friendship and for always being there for me. Your presence in my life is a true blessing.

I would also like to thank my funding body, King Abdullah International Medical Research Centre (KAIMRC), Ministry of National Guard, for the generous fellowship. In particular, I would like to thank the head of the centre, Dr. Ahmed Alaskar, for his fairness and continuous help and support. 
I dedicate this thesis to my family and my beloved cat for their constant support and unconditional love.

I love you all dearly. 


\section{Summary}

The first definitive haematopoietic stem cells (HSCs) produced during embryonic development are generated from a specialised subset of endothelial cells known as haemogenic endothelium. Recently, it was reported that the transcription factor Gata3 plays a dual role in the development of the sympathetic nervous system and the haematopoietic system. Specifically, Gata3 was demonstrated to be crucial for the production of HSCs through regulation of catecholamine production from the co-developing sympathetic nervous system. In addition, it was recently shown that Gata3 is expressed in the haemogenic endothelium and in haematopoietic progenitor cells. The aim of this thesis was therefore to examine if Gata3 is expressed and plays a role in the precursors from which HSCs arise and therefore would have an additional, more direct function in HSC emergence.

Using a Gata3-GFP reporter mouse line, we found that Gata3 is expressed in various cell types in the HSC microenvironment, including mesenchymal cells, endothelial cells, haematopoietic cells and sympathetic nervous system cells, with the expression being stage-dependant. In the endothelium, we discovered that the haemogenic activity is enriched in Gata3-expressing cells. Within haematopoietic cells, we found that Gata3 marks a specific stage along the developmental pathway towards the generation of definitive HSCs, and that Gata3-expressing haematopoietic cells are enriched for the most immature and stem cell-like progenitors.

To determine whether Gata3 plays an essential role in the production of HSCs from the endothelium, it was specifically knocked out in haemogenic endothelial cells using the Vec-Cre system. This revealed that Gata3 within the haemogenic endothelium plays a key part in the formation of haematopoietic progenitors and HSCs.

Finally, we used transcriptome analysis (RNA seq) to further investigate the function of Gata3 in the haematopoietic stem cell microenvironment and HSC 
precursors by comparing gene expression in the Gata3-positive and Gata3negative cell subsets in each compartment. The genes found to be enriched in Gata3-positive cells suggest that Gata3 plays a major role in the development and differentiation of various cells and systems, and implicate Gata3 as a cell cycle regulator.

In summary, we found that Gata3-expressing cells are enriched for haemogenic endothelium, crucial for haematopoietic stem and progenitor formation. Moreover, within these cells, Gata3 plays a key role in the endothelial to haematopoietic transition, and thus the formation of haematopoietic stem cells. 


\section{Table of Contents}

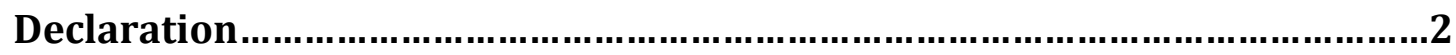

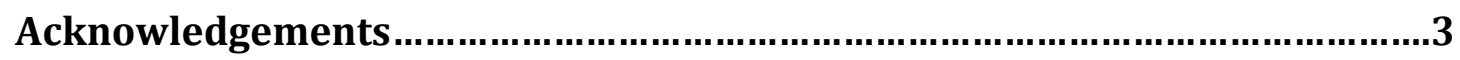

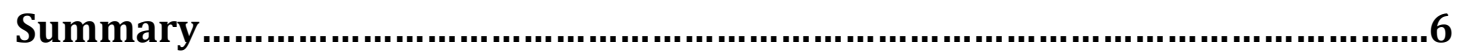

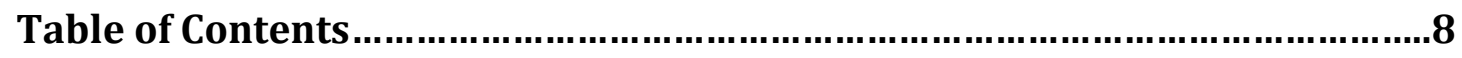

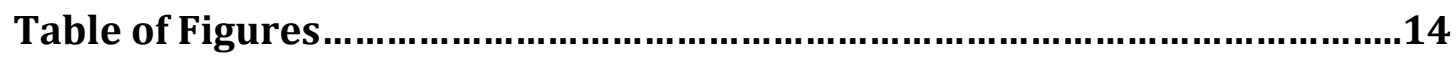

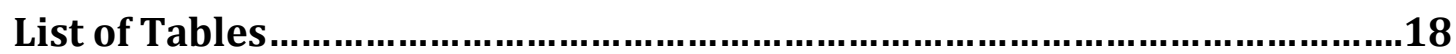

List of Abbreviations..................................................................................

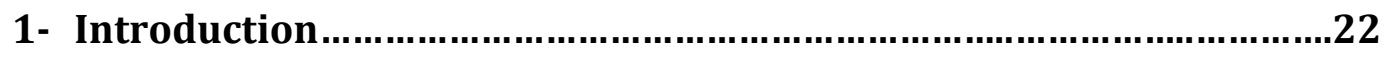

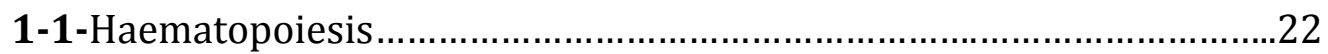

1-2-Developmental Origins of Blood Cells..........................................22

1-2-1-Primitive haematopoiesis....................................................23

1-2-2-Definitive haematopoiesis................................................24

1-3- dHSC Development and Specification ...........................................26

1-4-Haematopoietic Stem Cell Niche......................................................27

1-4-1- Components of the Niche............................................29

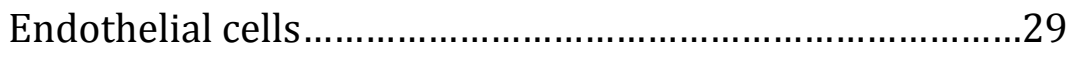

Mesenchymal Stromal/Stem Cells.....................................31

Sympathetic Nervous System......................................................34 
Gastro-Intestinal Cells

1-5- Haemogenic Endothelium

1-5-1-Generation of HSCs and Progenitors from Haemogenic Endothelium.

1-5-2-Characterisation of Haemogenic Endothelium .39

1-5-3-Types of Haemogenic Endothelium .41

1-5-4-Intrinsic regulators of EHT.

SCL/Tal1

Runx1 .41

HoxA3

Sox17.

1-6-1-Gata3 in development. .48

Skin and Hair .48

Kidney .48

Mammary gland .49

1-6-2-Gata3 in the Haematopoietic System .50 


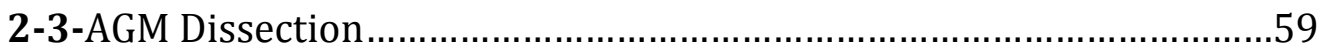

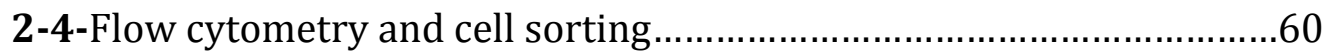

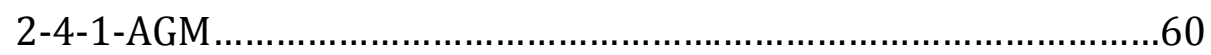

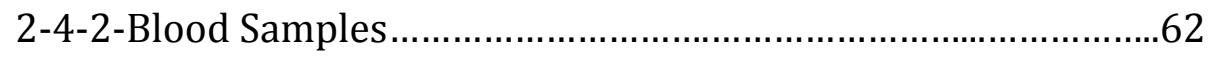

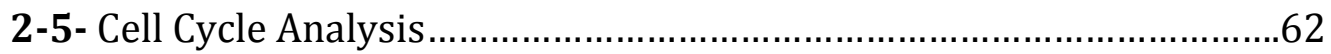

2-6- AGM explant culture..............................................................63

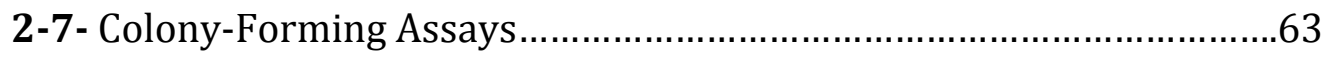

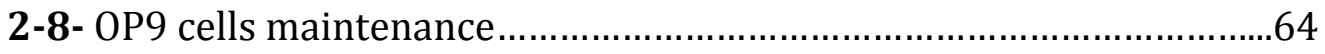

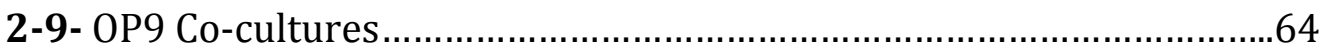

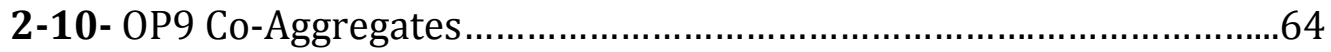

2-11- Transplantation Experiments..................................................65

2-12- Tissue preparation for Immunohistochemistry ............................66

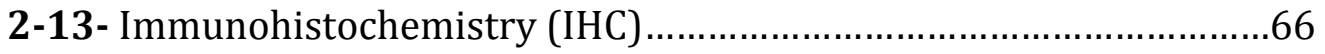

2-14- Statistical Analysis ........................................................... 67

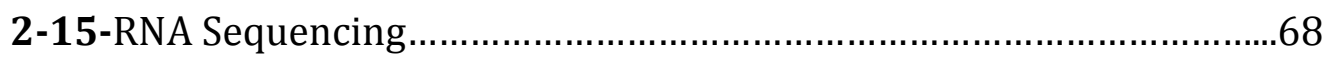

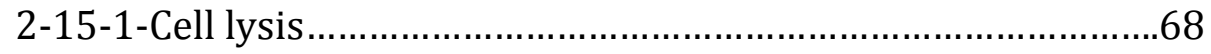

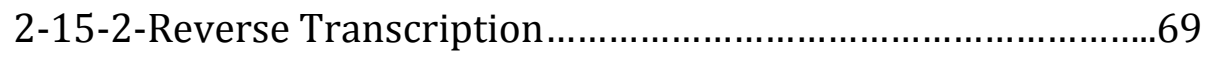

2-15-3- PCR Pre-Amplification ................................................. 70 
2-15-4- Quality Check of the cDNA Library.

2-15-5- Library Preparation.................................................... 71

2-15-6- Library Pooling and Clean-up...........................................72

2-15-7- Library Quality Check.................................................... 73

2-15-8- Library Quantification .................................................. 73

2-15-9- Sequencing \& Data Analysis................................................73

3- Gata3 expression marks haemogenic endothelium..............................75

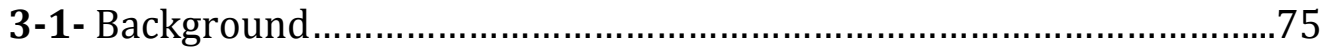

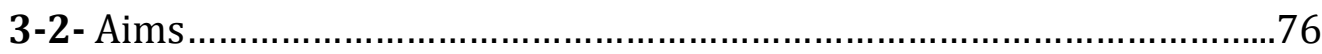

3-3-GATA-3 is expressed in various cell types in the E10.5 and E11.5 AGM. .77

3-3-1 E11.5 .77

3-3-2 E10.5 . .84

3-4- The haemogenic endothelium activity is enriched in the G3+ compartment. .86

3-5- G3+ progenitors are enriched for CFU-Mix potential, the most immature and stem cell-like progenitor. .90

3-6- Haematopoietic progenitors are restricted to the G3-HC compartment. .92

3-7- G3 marks specific stages along the developmental pathway towards the generation of dHSCs . .92

3-8-Discussion .96 
4-1- Background. .101

4-2- Aims .102

4-3- Gata3 deletion causes a reduction in haematopoietic progenitor cell counts in E10.5 and E11.5 AGMs in a dose-dependent manner. .103

4-4- G3+/- and G3-/- progenitors are unable to expand during explant culture .105

4-5- Gata3 within the HE plays a major role in haematopoietic progenitor formation. .107

4-6- The effect of Gata3 deletion from endothelium on haematopoietic repopulation 112

4-7- Discussion .114

5- Gata3 expression in the HSC microenvironment. 117

5-1- Background 117

5-2-Aims .118

5-3- Gata3+ and Gata3- cells segregate into distinct clusters.

5-4- The downregulated genes are associated with development and differentiation of various cells and systems

5-5- The upregulated genes are associated with migration, proliferation, development and apoptosis .125

5-6- Gata3 expression is associated with a more quiescent cell state.....128

5-7-Discussion 130 
6- Overall Discussion and Future Directions.

.134

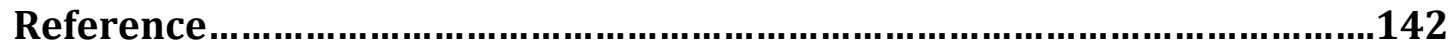




\section{Table of Figures}

Figure1-1: The haematopoietic tree .23

Figure1-2: A timeline view of haematopoiesis during mouse development.......24

Figure1-3: A schematic view of a cross section of the E11.5 AGM microenvironment. . .32

Figure1-4: The endothelial to haematopoietic transition. .39

Figure3-1: A section from a G3-GFP+ E11.5 embryo showing the co-staining between GATA3 and GFP

Figure3-2: FACS analysis showing a definitive spike in the GFP region in the AGM obtained from a G3-GFP E11.5 embryo compared to unstained AGM sample obtained from a wild-type littermate .78

Figure3-3: Flow cytometry analysis of G3-GFP+ samples from E11.5 AGMs, stained for CD45, CD34, PDGFRb and P75

Figure3-4: Section from a Gata3-GFP+ E11.5 embryo stained with Gata3-GFP, Th and CD34.

Figure3-5: Section from a Gata3-GFP+ E11.5 embryo stained with Gata3-GFP, PDGFRb and CD34

Figure3-6: Section from a Gata3-GFP+ E11.5 embryo stained with Gata3-GFP, Th and PDGFRb.

Figure3-7: Section from a Gata3-GFP+ E11.5 embryo stained with Gata3-GFP, Th, and P75.

Figure3-8: Section from a Gata3-GFP+ E11.5 embryo stained with Gata3-GFP, CD34, and Runx1 .83

Figure3-9: Gata3 expression in G3-GFP E10.5 AGMs. .85 
Figure3-10: Schematic diagram for experimental approach for OP9 coculture.

Figure3-11: CFU-C assay representing the progenitor counts of G3+EC, G3-EC, $\mathrm{HC}$ and MC/SNS cells plated into methylcellulose after OP9 co-culture.....

Figure3-12: Sorting Strategy for the OP9 co-culture . .88

Figure3-13: FACS analysis of the haematopoietic progeny produced by both G3+EC and G3-EC.

Figure3-14: CFU-C assay representing the progenitor counts of G3+EC and G3EC after OP9 co-culture .90

Figure3-15: G3+ progenitors are enriched for CFU-Mix potential, the most immature and stem cell like progenitor. .91

Figure3-16: CFU-C assay representing the progenitor counts of freshly sorted cells using the OP9 co-culture sorting strategy..... .92

Figure3-17: Schematic diagram for experimental approach for OP9 coaggregates

Figure3-18: Sorting Strategy for the OP9 co-aggregates. .94

Figure3-19: FACS analysis and CFU-C counts after OP9 co-aggregates. . .95

Figure3-20: Chimerism levels in transplanted animals from sorted cells G3+EC, G3-EC, G3+HC and G3-HC obtained from E9.5-E10.5 and E11.5 G3-GFP AGMs and co-aggregated with OP9-cells. .96

Figure4-1: Representative schematic of the experimental design of the CFU-C assay 103

Figure4-2: Bar graph shows total number of colonies and colony types obtained per $1 \times 10^{5}$ directly cultured E11.5 AGM cells. . .104

Figure4-3: Bar graph shows total number of colonies and colony types obtained per $1 \times 10^{5}$ directly cultured E10.5 AGM cells. . .105 
Figure4-4: Bar graph shows total number of colonies and colony types obtained per $1 \times 10^{5}$ cultured E11.5 AGM cells after an explant step. .106

Figure4-5: Bar graph shows total number of colonies and colony types obtained per $1 \times 10^{5}$ cultured E10.5 AGM cells after an explant step. 107

Figure4-6: Representative schematic of the experimental design of CFU-C assay 108

Figure4-7: Bar graph shows total number of colonies and colony types obtained per $1 \times 10^{5}$ directly cultured E11.5 AGM cells. . .109

Figure4-8: Bar graph shows total number of colonies and colony types obtained per $1 \times 10^{5}$ directly cultured E10.5 AGM cells. .110

Figure4-9: Bar graph shows total number of colonies and colony types obtained per $1 \times 10^{5}$ cultured E11.5 AGM cells after an explant step.

Figure4-10: Bar graph shows total number of colonies and colony types obtained per $1 \times 10^{5}$ cultured E10.5 AGM cells after an explant step .111

Figure4-11: PCR for colonies picked from CFU-C assays derived from E10.5 explant cultured AGM cells.

Figure4-12: Representative schematic of the experimental design of the transplantation assays

Figure4-13: Levels of chimerism from transplanted AGMs

Figure5-1: RNA-Sequencing sorting strategy.

Figure5-2: PCA showing the distribution of all Gata3 expressing populations in contrast to Gata3- populations. .120

Figure5-3: PCA showing the clustering of Gata3 expressing populations in contrast to their G3- counterpart

Figure5-4: Venn diagrams showing the overlapping genes between the different Gata3 expressing populations 
Figure5-5: G0 terms enriched in the downregulated genes in Gata3+ cells......124

Figure5-6: G0 terms enriched in the upregulated genes in Gata3+ cells...........126

Figure5-7: Cell cycle phase distribution of Gata3+ and Gata3- cells in the different cell populations in the AGM 129 


\section{List of Tables}

Table1: Genotyping primers and PCR conditions

Table2: Antibodies Fluorochrome and concentration for FACS

Table3: IHC Antibodies and concentration

Table4: List of cyclin dependent kinases (Cdk) and their inhibitors (Cdkn) found amongst upregulated genes and downregulated genes in different populations of G3+ cells 128

\section{List of Abbreviations}

7AAD: 7-aminoactinomycin D

AGM: Aorta gonad mesonephros

AGM AKT-ECs: AKT activated AGM derived endothelial cells

BM: Bone marrow

BMP4: Bone morphogenic protein 4

CAR: Cxcl12-Abundant 'Reticular' cells

CAR cells: CXCL-12-abundant reticular cells

Cdk : Cyclin dependent kinases

CD34: Hematopoietic progenitor cell antigen

CD41: Integrin alpha-IIb 
CD43: Leukosialin

CD45: Lymphocyte common antigen

CD117: c-Kit receptor

CMP: Common myeloid progenitor

Cdk: Cyclin-dependent kinase

cDNA: Complementary DNA

CFU-C: Colony forming unit-cell

CFU-M: Colony forming unit-macrophages

CFU-Mix: Colony-forming unit-mix

CFU-GM: Colony forming unit-granulocytes/macrophages

DAPI: 4', 6-diamidino-2-phenylindole

E: Embryonic day

ee: Embryo equivalent

EHT: Endothelial to haematopoietic transition

EMP: Erythro-myeloid progenitor

DNA: Deoxyribonucleic acid

DNase: Deoxyribonuclease

D0: Dorsa aorta

DOPAC: 3,4-dihydroxyhenyl acetic acid

dHSCS: definitive HSC

FACS: Fluorescence-Activated Cell Sorting

FL: Fetal liver 
G1: Gap 1 phase (cell cycle)

G2: Gap 2 phase (cell cycle)

Gata1: GATA Binding Protein 1

Gata2: GATA Binding Protein 2

Gata3: GATA Binding Protein 3

Gata4: GATA Binding Protein 4

Gata5: GATA Binding Protein 5

Gata6: Transcription factor GATA-6

GFP: Green Fluorescent Protein

HE: Haemogenic endothelium

HSPCs: Hematopoietic stem and progenitor cells

HSC: Haematopoietic stem cell

Igf-2: Insulin like growth factor

Il3: Interleukin 3

Il6: Interleukin 6

Il7: Interleukin 7

LMPP: Lymphoid primed multipotent progenitor

LT-HSC: Long term haematopoietic stem cell

LPs: Lymphoid progenitors

M: Mitosis phase (cell cycle)

MEP: Megakaryocyte-erythrocyte progenitor

MPP: Multipotent progenitor 
MSCs: Mesenchymal Stromal/Stem Cells

NO: Nitric oxide

qPCR: Quantitative real-time PCR

RNA: Ribonucleic acid

RNase: Ribonuclease

Runx1: Runt-related transcription factor 1

S: Synthesis phase (cell cycle)

Scf: Stem cell factor

ST-HSC: Short term haematopoietic stem cell

P75: Ngfr: Nerve Growth Factor Receptor

PAS: Para-aortic splanchnopleura

PBS: Phosphate-buffered saline

PCA: Principal Component Analysis

PCR: Polymerase Chain Reaction

PDGFRb: Platelet-derived growth factor receptor beta

PFA: Paraformaldehyde

Th: Tyrosine hydroxylase

Thpo: Thrombopoeitin

Tnfa: Tumour necrosis factor a

TEB: Terminal end buds

VE-Cad: Vascular Endothelial Cadherin, Cdh5, (Cd144)

YS: Yolk sac 


\section{1-Introduction}

\section{1-1-Haematopoiesis:}

Haematopoiesis refers to the formation of blood cellular components. All these components originate from the pluripotent haematopoietic stem cell (HSC), which is the foundation of this process.

The haematopoietic system is hierarchical, with the multipotent HSC being the mother cell that gives rise to and differentiates into multipotent and unipotent intermediates, resulting in the production of functional mature blood cells (Figure1-1) (Durand: 2005).

HSCs are crucial to maintaining haematopoiesis throughout life. Therefore, they have unique characteristics that distinguish them from other more mature cells: (i) self-renewal ability; (ii) high proliferation ability; (iii) long-term activity; and (iv) potential to differentiate into all blood lineages (Durand: 2005). All these characteristics make those cells the most clinically relevant cells for transplants.

\section{1-2-Developmental Origins of Blood Cells:}

The haematopoietic system is characterised by three different waves during development that can be broadly divided into primitive haematopoiesis (first wave) and definitive haematopoiesis (waves 2 and 3). 


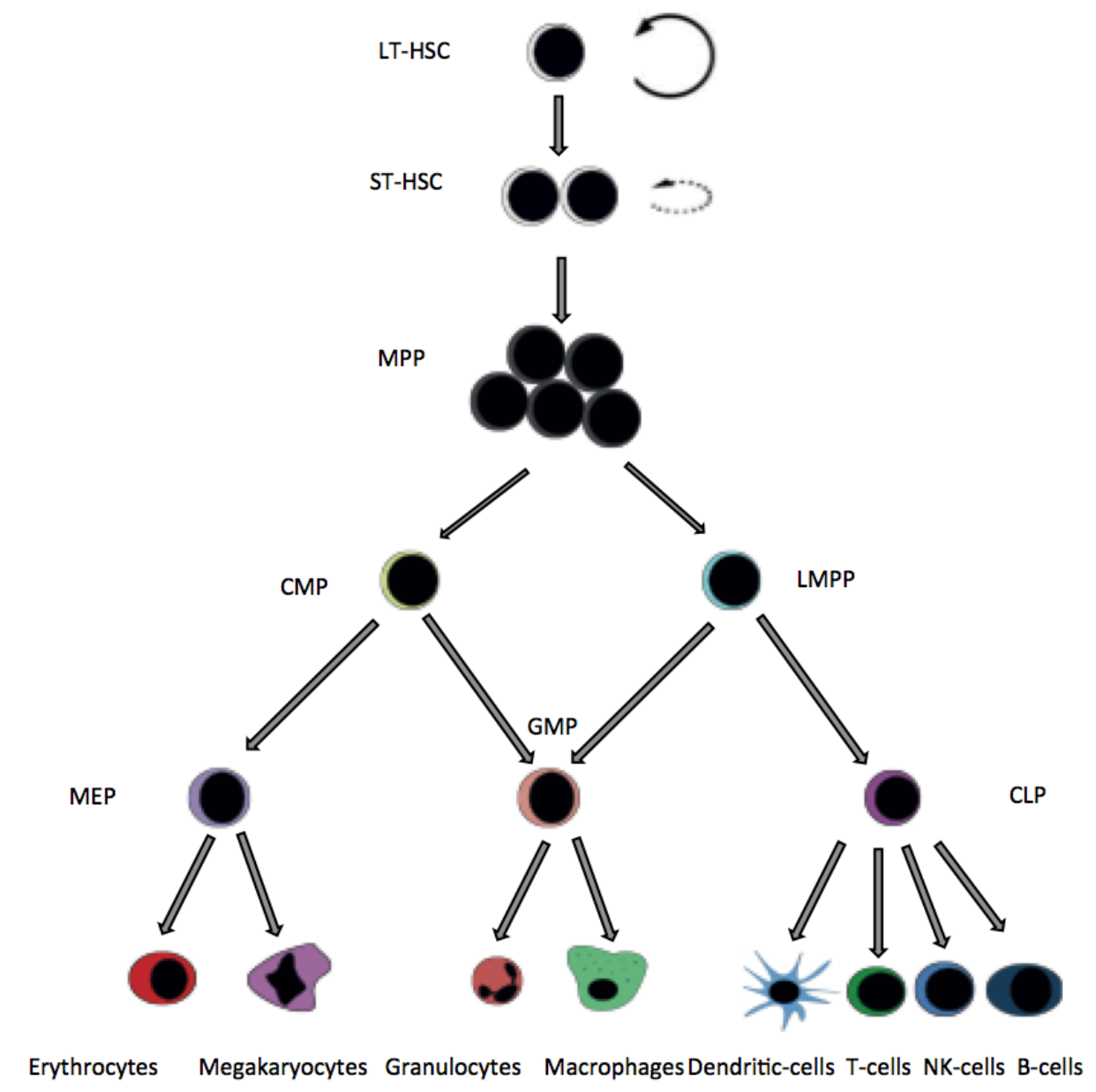

Figure 1-1: The haematopoietic tree: Long-Term HSCS (LT-HSCS) can self renew and give rise to all haematopoietic cells in the adult for the entire life span. HSCs can also give rise to Short-Term HSCs (ST-HSCs), which are able to reconstitute the blood for a limited time, and multipotent progenitors (MPP). These MPP have the ability to differentiate into lymphoid primed multipotent progenitors (LMPP) or common myeloid progenitors (CMP). The CMP then have the ability to develop into either megakaryocyte-erythrocyte progenitors (MEP) to ultimately give rise to red blood cells and platelets, or into granulocyte-macrophage progenitors (GMP), which then develop into granulocytes and macrophages. LMPP also have the ability to give rise to GMP, in addition to common lymphoid progenitor (CLP) to produce T-cells, B-cells, dendritic cells and natural killer cells (NK-cells). Adapted from (Mehta and Baltimore: 2016).

\section{1-2-1-Primitive haematopoiesis:}

Primitive haematopoiesis starts at E7.5 in the murine yolk sac mesoderm.

Based on the needs of the developing embryo, this wave is 


\begin{tabular}{l|l|l|l|l|l|l|l|l|l|l|l|}
\hline E7 & 8 & 9 & 10 & 11 & 12 & 13 & 14 & 15 & 16 & $\ldots$ & Birth
\end{tabular}

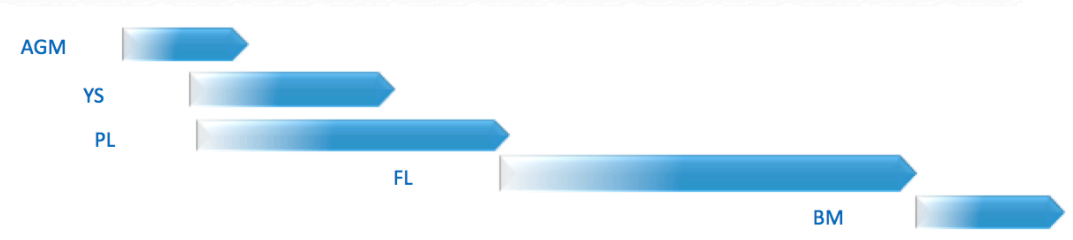

HSC Generation

Figure 1-2: A timeline view of the third wave of haematopoiesis during mouse development, characterised by HSC generation shown in blue. YS: yolk sac, FL: foetal liver, AGM: aorta gonad mesonephros, PL: placenta, BM: bone marrow. Adapted from (Ottersbach: 2010.)

responsible for the rapid production of transient erythroid cells. These cells have a higher affinity for oxygen binding, enter the circulation around E8.5 at the onset of foetal heart beating, mature within the circulation, and persist in the circulation until a few days after birth (Kingsley: 2004, Kingsley: 2006; De la Chapelle: 1969). This first wave gives rise to primitive erythrocytes, macrophages, and megakaryocytes.

\section{1-2-2-Definitive haematopoiesis:}

Shortly after the primitive wave of haematopoiesis in the yolk sac, definitive haematopoiesis initiates, generating lineage-restricted multipotent haematopoietic progenitor cells that exhibit adult-type morphology (second wave). Around E8.5, committed progenitors originate in the yolk sac and enter 
the circulation to seed the liver. These progenitors are generated during different times and at different haematopoietic sites in the mouse embryo. These places include the yolk sac, para-aortic splanchnopleura (PAS)/AGM, placenta, umbilical and vitelline arteries, and the heart. These progenitors then seed the liver, where they differentiate into the first definitive type of erythro-myeloid progenitors (EMPs) that circulate the embryo. These progenitors then generate definitive megakaryocytes, erythrocytes, macrophages, and other myeloid lineages (McGrath: 2015).

This wave also includes early $\mathrm{T}$ and B progenitors, which are produced in the yolk sac and para-aortic splanchnopleura (Yoder: 1997); and immunerestricted and lymphoid-primed progenitors that also originate from the yolk sac (Boiers: 2013).

The first definitive HSCs (dHSCs) emerge in the dorsal aorta within the aorta-gonad-mesonephros (AGM) region at E10.5 initiating the third wave (Figure 1-2). dHSC generation has also been reported in the head of E10.5-E11.5 embryo (Li: 2012). At E11.5 one dHSC is found in each of AGM, placenta, extraembryonic arteries, yolk sac and head (de Bruijn: 2000; Gekas: 2005; GordonKeylock: 2013; Kumaravelu: 2002; Li: 2012; Mikkola: 2005; Müller: 1994; Ottersbach and Dzierzak, 2005; Robin: 2009; Yoder: 1997). From then on, HSCs seed the foetal liver and dHSCs start to appear in the embryonic circulation. The liver then becomes the major site of haematopoiesis for the embryo until birth.

In this context, developmental haematopoiesis, the term dHSC refers to a HSC that can give rise to long-term haematopoiesis in an irradiated adult wild- 
type recipient directly upon transplantation without any culture steps (Ottersbach: 2010; Muller: 1994; Medvinsky: 1996; Medvinsky: 2011).

\section{1-3- dHSC Development and Specification:}

Definitive HSCs that give rise to the whole adult hematopoietic system emerges by late E10.5-E11.0 (Dzierzak and Speck: 2008; Medvinsky: 2011). Over the years, a number of theories have been proposed in order to explain how HSCs are generated in the dorsal aorta (de Bruijn: 2002), the most widely accepted indicating that the HSC lineage originates from the endothelium (Bertrand: 2010; Chen: 2009; de Bruijn: 2002; Kissa and Herbomel: 2010; Zovein: 2008: Jaffredo: 1998).

The HSC lineage shares Ve-cadherin with endothelial cells, which is then downregulated during development (Kim: 2005; Taoudi: 2005). Early in development, the hematopoietic progenitors express CD41 and only become CD45+ later in the development (Mikkola: 2003). Then, these cells undergo rapid developmental changes that are marked by the upregulation of CD41 in type I pre-HSCs: Ve-Cad+CD41lo CD45-, that is followed by upregulation of CD45 in type II pre-HSCs: Ve-Cad+CD45+ before finally becoming dHSCs (Rybtsov: 2011; Taoudi: 2008).

More recently, a new stage of the HSC lineage has been identified: the proHSCs. These cells differ from the subsequent type I and II pre-HSCs in that they 
require a longer culture period, 7 days compared to 4 days in pre-HSCs, and they also do not express CD43 (Rybtsov: 2014).

To sum it up, all three populations, pro-HSCs and pre-HSCs I and II, are Ve-Cad+CD41+c-Kit+ and can be further divided based on their CD43 and CD45 expression: the less mature pro-HSCs are CD43-CD45-, type I pre-HSCs are CD43+CD45-, and the more mature type II pre-HSCs are CD45+CD43+. More recently, CD27 has also been identified as a marker for pre-HSCs type II and dHSCs (Li: 2017).

It should be noted here that haematopoietic progenitors (CFU-Cs) develop in parallel with the HSC lineage and are available in the major arteries, around 500-1000 progenitors with lymphoid potential, and they also share markers with both type II pre-HSCs and dHSCs. CFU-Cs expresses CD43+ and CD41+ early in development, and later on they start to express CD45 as well. These CFU-Cs are not able to repopulate irradiated adult recipients, and unlike pro/pre HSCs, they are able to give rise to colonies in CFU-C assay. (Rybtsov: 2014; Lin: 2014; Li: 2014; Mikkola: 2003).

\section{1-4-Haematopoietic Stem Cell Niche:}

Stem cell behaviour is controlled by integration of intrinsic and extrinsic factors that are supplied by the surrounding microenvironment. Stem cell niche refers to the regulatory microenvironment within a specific anatomical location where stem cells reside. This microenvironment is composed of cellular and 
acellular compartments, which interact with each other and with the stem cells to regulate their proliferation, differentiation, localization and survival (Jones: 2008).

In the haematopoietic system, this niche is mainly composed of stromal cells and soluble factors that are secreted by these cells. These stromal cells provide HSCs with cell-cell anchorage and through cell-cell signalling control their survival, proliferation, differentiation and trafficking. The soluble factors can regulate HSCs directly, or indirectly by acting on the regulatory microenvironment, which in turn acts on HSCs.

The HSC has a dynamic nature; it has the ability to adapt and respond to different cues based on the physiological needs of the body. Given the niche's role in regulating HSC activity, it should be expected that the adult HSC niche and the embryonic HSC niche would have both common and unique characteristics based on the needs of the body at a specific stage and time.

During development, the niche of the HSCs can be found at different anatomical locations based on the needs of the developing embryo, whereas the adult niche is the bone marrow (BM). The different anatomical locations of the embryonic niche at different time points serve to support different functions and needs in the developing embryo. Some of these niches, such as AGM, yolk sac and placenta, have a transient nature. However, the main functions of the adult HSC niche (BM) are to maintain highly quiescent HSCs, control HSC differentiation and mobilisation into the blood (Ottersbach: 2009). 


\section{1-4-1- Components of the Niche:}

Cells in the HSC niche can regulate HSC activity either through physical means or by producing chemicals to regulate haematopoiesis. This regulation can be done either by cells that are found in the immediate microenvironment of HSCs, endothelial and stromal cells, or by neighbouring cells of other developing systems.

A gene expression profiling study of the AGM at the time of HSC emergence has shown that the upregulated genes were not restricted to the ones involved directly in haematopoiesis, but also included genes that are involved in the development of the nervous, vascular, muscular and skeletal systems (Mascarenhas: 2009). Given that, and considering the close proximity of systems to one another in the AGM, the possibility that signals from one system might influence the development of another seems plausible.

\section{Endothelial cells:}

Recent studies have suggested that HSCs and progenitors are derived from a subset of endothelial cells that is called the haemogenic endothelium (Figure 1-3) (Bertrand: 2010; Chen: 2009; de Bruijn: 2002; Kissa and Herbomel: 2010; Zovein: 2008). The process by which the blood cells are made, is called endothelial-to-haematopoietic-transition (EHT) (Figure 1-4) (Lam: 2010; Kissa and Herbomel: 2010). Both the haemogenic endothelium and EHT will be discussed further in the next section. 
Endothelial cells themselves have the ability to support HSCs in in-vitro cultures (Imanirad: 2014), and transplanting them into irradiated mice improved haematopoietic stem cell reconstitution in those mice (Salter: 2009).

Kobayashi et al. have shown that when endothelial cells of adult mice are activated through differential recruitment of Akt and P42/44 mitogen-activated protein kinase (MAPK) signalling pathways, it balances the self-renewal of LTHSCs and differentiation of hematopoietic stem and progenitor cells (HSPCs) (Kobayashi: 2010).

Another study activated AGM EC with AKT to generate AKT-activated AGM-derived endothelial cells (AGM AKT-ECs), and thus forming a vascular niche that is suitable for co-culture. These cells were able to create an in vitro niche that was able to recapitulate embryonic HSC specification and amplification, i.e. it promoted the induction of engrafting, non-haematopoietic precursors and thus, inducing endothelial to haematopoietic transition in vitro (Hadland: 2015).

More recently, Li et al. (2017) have demonstrated that co-culturing preHSCs with AKT activated endothelial cells (AKT) supported the maturation of pre-HSCs into HSCs (Li: 2017).

In the spleen and bone marrow, many HSCs are associated with sinusoidal endothelium and to a lesser extent, some of the HSCs were associated with endosteum (Kiel: 2005). 
Reprogramming studies have illustrated that the expression of four transcription factors (FOSB, GFI1, RUNX1, and SPI1) in human endothelial cells or in adult mouse endothelial cells were sufficient to generate immunocompetent HSPCs (Sandler: 2014; Lis: 2017)

Furthermore, endothelial cells, in addition to intra-aortic clusters and smooth muscle cells, produce Insulin like growth factor (Igf2), which is known to promote the production of more primitive progenitors and erythroid progenitors in the AGM (Mascarenhas: 2009).

Nitric oxide (NO) is also produced by the endothelium in response to shear stress, which in turn increases the production of haematopoietic and progenitor cells (North: 2009; Adamo: 2009).

\section{Mesenchymal Stromal/Stem Cells:}

Mesenchymal stem/progenitor cells are multipotent stem cells that have the ability to differentiate into cells of adipogenic, chondrogenic and osteogenic lineages (Dennis and Charbord: 2002). During development, these cells first appear in the AGM at the time of HSCs emergence (Figure 1-3)(Mendes: 2005). At that stage, both MSCs and HSCs share similar patterns: i) they appear in waves; primitive and definitive, ii) they co-localise in the AGM and other major haematopoietic sites, iii) they follow the same developmental time-line. However, despite their co-localisation in haematopoietic harbouring sites and their occurrence in blood circulation, their presence at this stage is independent of haematopoietic development (Mendes: 2005). 


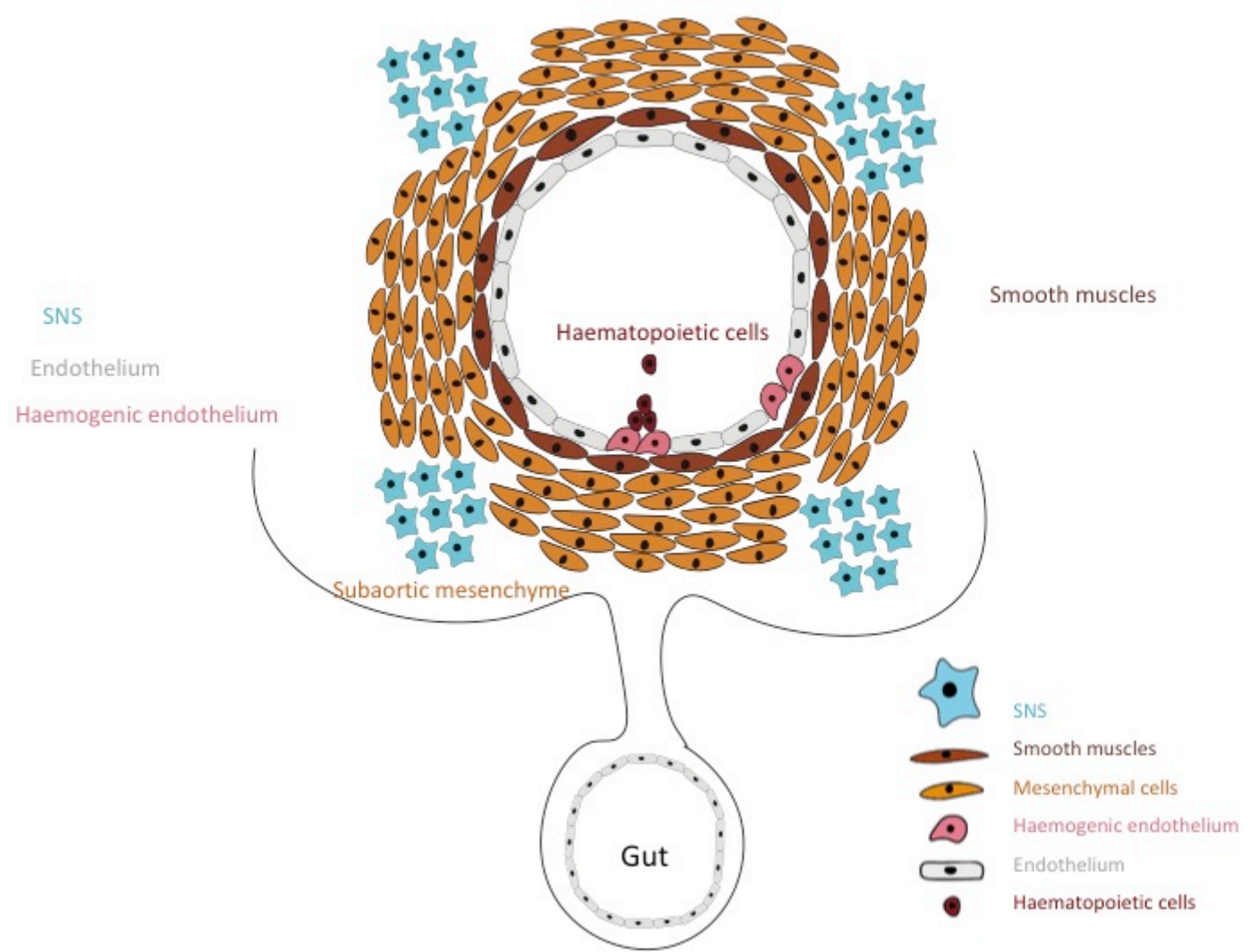

Figure 1-3: A schematic view of a cross section of the E11.5 AGM microenvironment showing different cell types that make up the HSC niche in the AGM. The haemogenic endothelium cells, pink cells, are lining the wall of the dorsa aorta along with non-haemogenic endothelium. The haematopoietic cells bud out from the haemogenic endothelium and detach to become a haematopoietic cell

MSCs refer to a heterogeneous group of cells in the adult mouse. Some of these have been associated with HSC maintenance. The CXC chemokine ligand CXCL-12-abundant reticular (CAR) cells are a subset of mesenchymal cells that has been implicated as a niche for HSCs. When those cells were deleted temporarily, the production of the cytokines stem cell factor (Scf) and CXCL-12 ceased and, as a result, HSCs were reduced in size and numbers (Omatsu: 2010). In addition, Sugiyama et al. (2006) has shown that when the receptor for CXC chemokine ligand CXCL-12 (CXCR4) was deleted, it resulted in a sharp decline of 
HSC counts, and that CXCL12-CXCR4 signaling regulates the quiescent HSC pool (Sugiyama: 2006).

Leptin receptor (Lepr)-expressing perivascular cells are also a subgroup of mesenchymal cells that is involved in HSC maintenance. When Scf was deleted from those cells or endothelial cells, HSCs were depleted from the bone marrow (Ding: 2012)

Similar to CAR and Lepr cells, there is also another subset of mesenchymal cells that has been linked to HSC maintenance; the filament protein nestin-expressing cells. These cells are also involved in CXCL12-CXCR4 signalling, and are capable of producing Scf (Mendez-Ferrer: 2010).

Stem cell factor (Scf) is a cytokine that binds to the C-kit receptor (CD117), and is expressed by all HSCs. It can exist both as a transmembrane protein or a soluble factor and is mainly expressed in the ventral stroma and the urogenital ridges in the AGM. By E9.5 Scf is clearly expressed and that expression is highly upregulated by E10.5. Scf was found to be essential for initiating the HSC lineage development as it regulates the transition of pro-HSCs to pre-HSCs. In addition, Scf is a strong trigger for dHSC development from both type I and type II pre-HSCs (Rybtsov: 2014; Souilhol: 2016).

In addition, thrombopoietin (Thpo) is expressed in the ventral mesenchyme of E11 AGMs. When it was added to AGM explant cultures, it enhanced the haematopoietic progenitor and HSCs production by regulating their survival and proliferation. (Mascarenhas: 2016). 
Bone morphogenic protein 4 (BMP4) is a cytokine that is produced by the ventral mesenchyme in the developing embryo, which was reported to support haematopoietic and more committed progenitor cell differentiation (Chan: 2013).

\section{Sympathetic Nervous System:}

The presence of a normal functioning sympathetic nervous system (SNS) is essential for the production of HSCs (Figure 1-3). Using a Gata3 total knockout

mouse model, Fitch et al. have shown that when Gata3 was deleted from embryos, the cells of the SNS failed to differentiate and did not produce Tyrosine Hydroxylase (Th), a rate-limiting enzyme for catecholamine synthesis that is expressed in sympathoadrenal cells, and hence failed to produce catecholamines. Those embryos had functionally and phenotypically impaired HSC production compared to their wild-type littermates. Adding catecholamines to these embryos via the drinking water of the mother rescued HSC numbers (Fitch: 2012).

Moreover, when AGMs from a Th knockout mouse line, which cannot produce catecholamines, and wild-type AGMs that were placed in an explant culture in the presence of a tyrosine hydroxylase inhibitor, were used for transplantation assays, the results showed a marked reduction in repopulation ability.

Furthermore, AGM HSCs express the beta2-adrenoreceptor, which binds catecholamines, indicating that catecholamines released from the developing SNS can act directly on emerging HSCs in the AGM (Fitch: 2012). 
The ablation of adrenergic neurotransmission, either genetically or pharmacologically, has shown that signals from the SNS supress osteoblast function and control the attraction of HSPCs to their niche (Katayama: 2005). Catecholamines were also shown to promote HSC proliferation directly via the beta2-adrenoreceptor (Spiegel: 2007).

In the adult mouse, Mendez-Ferrer et al. have reported that the circadian release of HSCs from BM is regulated by norepinephrine (NE) that is locally released from nerve terminals of the SNS (Mendez-Ferrer: 2010).

\section{Other Haematopoietic Cells:}

Given the responsive and dynamic nature of HSCs, it is possible that more developed haematopoietic progeny might affect HSC behaviour in terms of regulating the supply and demand of a certain blood type in normal haematopoiesis and stress conditions.

The regulation of HSCs by their mature progeny has been demonstrated in several studies. One of the studies used mice with mutant Myb alleles that had defective megakaryopoiesis, and showed that the abnormal increase in platelet production altered thrombopoietin levels, which in turn resulted in functionally impaired HSCs that declined in numbers with age (de Graaf: 2010).

Another study demonstrated that the regulation of HSCs by mature progeny can happen through the stromal niche. BM macrophages were found to interact with Nestin+ MSCs to promote the retention of HSCs in the BM (Chow: 2011). 
During development, the primitive myeloid population was found to regulate the numbers of haematopoietic stem and progenitor cells in the AGM region by contributing to the local inflammatory response. In a study by Li et al. (2014), mouse embryos deficient in interferon-g (IFN-g) or IFN-g and interferona (IFN-a) had lower lymphoid progenitor counts, and the number of transplantable HSCs was lower in embryos lacking IFN-g (Li: 2014).

Furthermore, in the absence of infection, proinflammatory signaling was reported to be involved in the HSC program development. Primitive neutrophils were identified by Espin-Palazon et al. as a major source for tumour necrosis factor a (Tnfa). The loss of either neutrophils or Tnfa resulted in the loss of HSCs. It should be noted that Tnfa is also produced by endothelial cells, and that it can also contribute to the inflammatory signaling from that cell compartment (EspinPalazon: 2014).

\section{Gastro-Intestinal Cells:}

Haematopoietic activity has been associated with the ventral side of the embryonic dorsal aorta in many organisms. Previously, it has been reported that the ventral region of the dorsal aorta in the mouse contains most of the HSC activity at E11.5, and is able to increase HSCs in explant culture. Furthermore, it is the only domain that is able to generate HSCs in E10.5 explant cultures (Taoudi: 2007).

In another study (Peeters: 2009), the ventral tissues of the dorsal aorta demonstrated a positive influence on AGM HSC generation, whereas dorsal tissues had a suppressive effect. Specifically, in the study from Peeters et al. 
(Peeters: 2009), it was shown that when AGMs from early E10 or gut explants were cultured alone, there was no output from either of them; however, when they were cultured together in an early E10 AGM:gut explant, HSCs were generated after 3 days in culture. This could be attributed to the presence of certain signaling pathways in the ventral region of the dorsal aorta. One such pathway is the Hedgehog pathway that originates from the developing gut at E10.5. Hedgehog proteins also regulate the expression of the gene cascades that are required for arterial development, including Vegf, Notch, Ephrin2a and Runx1 (Gering and Patient: 2005).

Explant culture studies then revealed that signals from the gut induce HSCs from the AGM, and this induction was blocked when an anti-hedgehog antibody was added to the culture medium. In addition, the expression of one of the intracellular signalling components, Gli1, was at its highest within the subaortic mesenchyme, thus indicating that hedgehog signals from the gut act on the mesenchyme to influence HSC generation (Peeters: 2009).

IL-3 is a cytokine that is mainly produced by the gut in the developing embryo. IL-3 was found to be a potent inducer of dHSC function in the E10.511.5 AGM region; it increases HSC survival, proliferation, and repopulation capacity (Robin: 2006).

1-5- Haemogenic Endothelium:

\section{1-5-1-Generation of HSCs and Progenitors from Haemogenic Endothelium:}


Both long-term HSCs and progenitors originate from the haemogenic endothelium (HE); a specialised subset of endothelium (Bertrand: 2010; Chen: 2009; de Bruijn: 2002; Kissa and Herbomel: 2010; Zovein: 2008). The idea of blood cells emerging from haemogenic endothelium was proposed 100 years ago based on the observation of embryos using simple microscopy (Jordan: 1916; Emmel: 1916).

Given the association of haematopoietic intra-aortic clusters with the endothelial lining of the dorsal aorta, it was thought that HSCs were derived from these endothelial cells. By the start of the $20^{\text {th }}$ century, the term haemogenic endothelium was used to describe the intra-aortic clusters that were believed to have endothelial origins (Jordan: 1916; Jordan: 1917; Emmel: 1916).

The embryonic dorsal aorta has been known to be associated with haematopoietic cells for some time. Clusters of haematopoietic cells that coexpress endothelial markers are attached to both the dorsal and the ventral side of the aortic endothelium in mouse embryos (Ivanovs: 2014; Ciau-Utiz: 2014; Garcia-Porrero: 1998; Tavian: 1996; North: 1999). However, only the ventral side of the dorsal aorta have shown to contain HSCs (Taoudi: 2007).

The process by which endothelial cells commit to the haematopoietic fate is known as endothelial to haematopoietic transition (EHT). Recently, timelapsing imaging studies have visualised this transition of endothelium to blood in-vitro (Lancrin: 2009; Eilken: 2009), in ex-vivo explant cultures (Boisset: 2010) and in the zebrafish embryo in vivo (Bertrand: 2010; Kissa: 2010; Lam: 2010). In the zebrafish in vivo imaging studies, this process was shown to start with the 
endothelial cell bending out and rounding up to finally detach from the vascular wall to become a free-moving haematopoietic cell (Figure 1-4 A and B). The factors that regulate the process of EHT include blood flow-induced sheer stress, signalling pathways, and transcriptional regulators (described further below). However, the underlying mechanism of this process and the accompanying structural changes are poorly understood (Kissa: 2010).

A

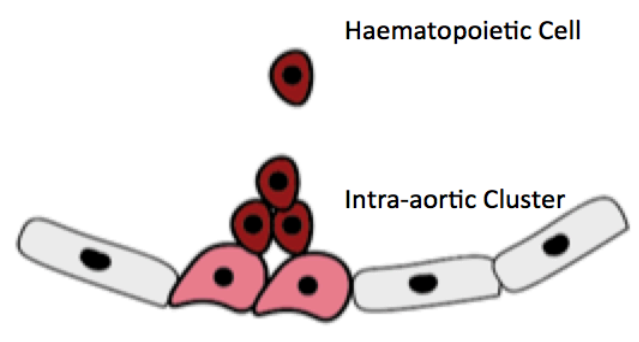

HE

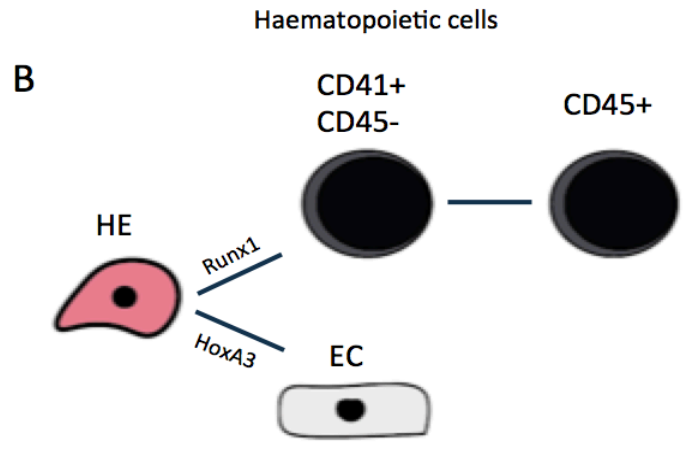

Structural Endothelium

Figure 1-4: The endothelial to haematopoietic transition; (A) The haematopoietic cells bud out from the haemogenic endothelium and detach to become a free-living haematopoietic cell. (B) the haemogenic endothelium can develop into either a haematopoietic cell, which is Runx1-dependent, or into endothelial cells. HoxA3 maintains the endothelial program in the cells by antagonising the haematopoietic program (Adapted from Medvinsky: 2011)

\section{1-5-2-Characterisation of Haemogenic Endothelium:}

Haemogenic endothelium displays endothelial phenotype and morphology, and has the potential to form endothelial sheets and tubules, while also being able to give rise to haematopoietic progeny in culture. Defining and isolating the haemogenic endothelium has been difficult. This is due to the fact that expression of the endothelial markers CD31, Flk-1, and VE-Cadherin overlaps between haemogenic endothelium, HSCs, haematopoietic progenitors, 
and non-haemogenic endothelium (Nishikawa: 1998). Therefore, a combination of both endothelial markers and haematopoietic markers, such as CD41 (Corbel: 2002), CD45 (Ledbetter: 1979) and Ter119 (Kina: 2000), has been utilised to separate haematopoietic from endothelial cells; even more of a challenge has been to isolate haemogenic endothelium from non-haemogenic endothelium. (Swiers: 2013).

The core binding factor family member Runx1 is expressed in haemogenic arteries before HSC emergence. While endothelial-specific deletion of Runx1 did not affect haemogenic endothelium generation, no LT-HSCs were produced (Chen: 2009). Thus, Runx1 is essential for the EHT and was subsequently investigated as a tool for identifying HE (Swiers: 2010).

In their paper, Swiers et al. have managed to isolate the HE by developing a Runx1 +23 enhancer-reporter transgenic mouse model (23GFP) to investigate the EHT. By using functional and transcriptional assays on HE, identified as $23 \mathrm{GFP}+$ cells, it was demonstrated that the haematopoietic commitment of the HE starts at E9.5 while the cells are still embedded in the endothelial layer, and those cells lose their endothelial potential before HSC emergence. Thus, they have proposed a new model; an early onset model of haematopoietic programming and loss of endothelial potential for the HE, as opposed to what was believed before of the HE of having the potential to differentiate into either endothelial tubules or haematopoietic progeny. They also reported that Gata3 was expressed at higher levels in 23GFP+HE than in non-haemogenic endothelial (NHE) cells. 


\section{1-5-3-Types of Haemogenic Endothelium:}

Haemogenic endothelium appears at different times and different locations during development. However, it was still unclear if there were different types of haemogenic endothelium, one that generates HSCs and another that generates progenitors. Chen et al. demonstrated this in a study where they used CBFb-deficient embryos (Chen: 2011). CBFb is the hererodimeric partner of Runx1, and in its absence, embryos demonstrate the same phenotype as Runx1deficient embryos; they die at midgestation due to central nervous system haemorrhage and fetal liver anemia. Adding GFP-CBFb back to them by using two different tissue-specific expression cassettes, Tie2 and Ly6a, rescued the formation of erythro-myeloid progenitors (EMPs) and HSCs, respectively in $\mathrm{CBFb}$ null embryos. When the Tie2 cassette was used, GFP-CBFb was expressed in YS endothelium, endothelial cells of the dorsal aorta (DO), placenta and umbilical and vitelline arteries; however, only the EMPs were rescued, but not the HSCs.

Interestingly, when the Ly6a regulatory cassette was used, only HSCs were rescued but not EMPs. This indicates that EMPs and HSCs differentiate from distinct populations of haemogenic endothelial cells, with Ly6a expression specifically marking the HSC-generating haemogenic endothelium (Chen: 2011).

\section{1-5-4-Intrinsic regulators of EHT:}

\section{SCL/Tal1:}

The transcription factor SCL/Tal1 is essential for establishing the haematopoietic transcription program in haemogenic endothelium and 
repressing its cardiac fate. Using a transgenic mouse line, Van Handel et al. have shown that when SCL was deleted, the cardiac transcriptional program in YS endothelium was activated, indicating that SCL is required to prevent the misspecification of haemogenic endothelium to cardiomyogenic fate (Van Handel: 2012). Interestingly, SCL is not required for haematopoietic cell generation once the haemogenic endothelium is established, while Runx1 is crucial for haematopoietic cell generation, thus placing SCL upstream of Runx1 (Lancrin: 2009).

More recently, it was reported that SCL knockout does not bias cells towards cardiac fate. In single cell transcriptome experiment using a SCL (Tal1) knockout mouse line (Tal\%), Scialdone et al. have found that while the haematopoietic genes were downregulated, there was no upregulation of cardiac markers, thus indicating that while the primary function of SCL is the induction of the haematopoietic program, it doesn't directly inhibit the cardiac program (Scialdone: 2016).

\section{Runx1:}

A large amount of research has been dedicated to the transcription factor Runx1 given its crucial role as a master regulator of haematopoiesis, specifically its essential role in EHT (Figure 1-4 B).

The first studies reported that Runx1 activates the haematopoietic program while repressing the endothelial program (Jaffredo: 2005). More recently, Runx1 was found to regulate the positioning and integration of HE cells before haematopoietic cell release (Lie A Ling: 2014). Runx1 target genes Gfi1 
and Gfi1b regulate the loss of endothelial gene expression, as the first step in haematopoietic progeny formation from HE in EHT. Gfi1 and Gfi1b were able to trigger the downregulation endothelial markers in Runx1 null haemogenic endothelium derived from ES cells. Those Gfi1/Gfi1B transduced cells were able to undergo morphological changes that are similar to that of haematopoietic progeny and characteristic of EHT; i.e. generating a round cell that expresses CD41. However, these cells were incapable of producing haematopoietic colonies in vitro unlike wild-type CD41-expressing cells that are either isolated from embryos or from ES culture (Lancrin: 2012).

Interestingly, deleting both Gfi1 and Gfi1b from embryos resulted in the formation of CD41-expressing cells that maintained their endothelial gene expression and characteristics and continued to adhere to their cell layer in the YS. However, these cells can generate haematopoietic progenitors when disaggregated (Lancrin: 2012).

Using an ES cell line with an inducible form of Runx1 in a Runx -/background, a ChIP-sequencing study looked at the binding pattern of three known haematopoietic transcription factors (SCL, Fli1, and C/EBP $\alpha$ ) during EHT and compared the binding before and after Runx1 induction (Lichtinger: 2012). After Runx1 induction, the binding of those factors was similar to that found in haematopoietic cells more than to that in HE.

\section{HoxA3:}

The start of the haemogenic potential of the endothelium that lines the walls of the dorsal aorta is associated with a fine balance between the expression 
of HoxA3 and Runx1. HoxA3 restrains haematopoietic differentiation of endothelial cells while Runx1 promotes it (Figure 1-4 B). Loss and gain of function studies have shown that expression of HoxA3 is downregulated at the onset of Runx1 expression, and re-expressing HoxA3 in the earliest haematopoietic progenitors induces their reversion to endothelial cells (Iacovino: 2010). Another marker of haemogenic endothelium that was found downstream of HoxA3 is Sox17 (Iacovino: 2010).

\section{Sox17:}

Sox17 is expressed and required for the development of neonatal and fetal HSCs but not adult HSCs (Kim: 2007), and the conditional deletion of Sox17 from endothelial and haematopoietic progenitors using the Tie2-Cre mice, or only from the endothelial cells using the Vec-Cre system is embryonically lethal (Clarke: 2013; Kim: 2007). When Sox17 was over-expressed, it was found to reduce the expression of endothelial markers and expand a cell population that has haemogenic potential (Clarke: 2013).

Sox17 was identified as a key regulator of haemogenic endothelial development. Using a Sox17-GFP reporter mouse line, Clarke et al. have shown that Sox17 is expressed in haemogenic endothelium and emerging HSCs and that it is required for HSC development. In addition, Sox17 is also expressed in haemogenic endothelium generated in vitro. Sox17 acts through the Notch signalling pathway to regulate the development and expansion of haemogenic endothelium (Clarke: 2013). 


\section{1-6- Gata3:}

The GATA family of transcription factors are master regulators in development. All GATA transcription factors bind to a WGATAR DNA sequence motif at target genes to control their transcription through two zinc fingers; an $\mathrm{N}$-terminal and a C-terminal. Those fingers bind to different targets and each one of them has a unique function. The C-terminal zinc finger binds to the GATA consensus sites, while the $\mathrm{N}$-terminal zinc finger facilitates the interaction between the GATA transcription factor and other specific DNA sequences (Martin: 1990; Trainor: 1996; Fujiwara: 2009). In a crystallographic structural study by Bates et al. (2008) for GATA3, it was found that the GATA family members could bind to DNA by either homo or heterodimerizing, or directly by forming a dimer with other GATA factors through their two $\mathrm{C}$ zinc fingers. In a genome-wide ChIP-seq experiment, Fujiwara et al. (2009) reported that GATA proteins preferred binding to WGATAA sequences in vivo

Each GATA transcription factor is highly conserved across vertebrates. For example, GATA3 homologs are found in human, mouse, rat, chimpanzee, dog, chicken, frog, and zebrafish. GATA3 shares 97\% of its amino acid identity between mouse and human.

The GATA family has six members in vertebrates, and the disruption of each of the GATA genes, with exception of GATA5, in mice during development causes embryonic lethality. This family is grouped into haematopoietic (GATA1, GATA2, GATA3), and endodermal (GATA4, GATA5, GATA6) subgroups (Burch: 2004). 
All GATA factors have both distinct and common biological roles and biochemical characteristics, and all these transcription factors have a restricted expression pattern. However, to some extent, their functions are interchangeable (Burch: 2004). For example, Gata1, Gata2, Gata3 and Gata4 can activate interleukin-4 (IL4) and IL5 expression in T cells, which are classically target genes for Gata3, and repress the activation of interferon-g (Ifng) (Ranganath and Murphy: 2001). Moreover, Gata3 knock-in can partially rescue erythrocyte defects in Gata1 null mice. However, Gata3 cannot fully rescue the Gata1 null mice, indicating that each Gata factor maintains its unique functions (Tsai: 1998, Takahashi: 2000).

The first three of the GATA family play essential roles in the development and maintenance of the haematopoietic system; Gata1 induces megakaryocyte and erythrocyte development, while preventing granulocyte-monocyte and lymphoid commitment. It is also expressed in common lymphoid and myeloid progenitors, mast cells and eosinophils (Iwasaki: 2003; Harigae: 1998). Gata2 is necessary for the formation and function of HSCs (Tsai: 1997). Gata3 is crucial for multiorgan development and regulates tissue-specific differentiation, which will be discussed further below. Gata4, Gata5 and Gata6 are critical for the development of heart and lung, and are expressed in tissues that originate from mesoderm and endoderm (Molkentin: 2000).

The haematopoietic group within the GATA factors control each other's expression during development in different cells, and are capable of functioning consecutively during cell specification and lineage commitment in a process called the GATA switch. GATA switch refers to a process where one GATA factor 
is replaced by another GATA at the chromatin site. GATA switches occur at many functionally critical loci during development. Some of these GATA switches occur at genes that regulate haematopoiesis, such as Gata2, which indicates that it could control haematopoiesis (Bresnick: 2010). For example, Gata2 is one of the genes that are direct targets of Gata1. Gata2, in the absence of Gata1, can bind to its target region upstream of its own promoter, which activates transcription and induces histone acetylation. However, when Gata1 is expressed, Gata2 is displaced by Gata1 at its chromatin site, which activates erythropoiesis (Suzuki: 2013).

Transcription factors are often organized in multi-gene families and play essential roles in activating target genes of specific cell fates and in repressing target genes of alternate cell fates. The GATA family of transcription factors are such master regulators.

The GATA factors share common features: they contain two transactivation domains at the amino terminus, two zinc fingers at the carboxyl terminus and a conserved basic region that is located immediately after each zinc finger motif. GATA family members bind to a consensus DNA sequence (A/T) GATA $(A / G)$ in the promoters of genes to directly activate or repress expression of target genes. In addition, GATA factors recruit chromatin-remodelling complexes to remodel gene loci (Takemoto: 2002; Zhou and Ouyang: 2003; Yamashita: 2004).

At the amino acid level, the family members share varying degrees of homology. For example, while GATA2 and GATA3 are about 55\% homologous, GATA3 and 
GATA4 are only $20 \%$ homologous. However, the zinc finger motifs are about $80 \%$ homologous among all the six members.

Gata3 plays a major role in a variety of cells in developmental stage and in cell lineage specification. It is crucial for the development of several types of cells, tissues, and organs, including adipocytes (Tong: 2000), kidney (Grote: 2006), mammary gland (Asselin-Labat: 2007), skin (Kaufman, 2003; Kurek, 2007), and sympathetic nervous system (Lim: 2000).

\section{1-6-1-Gata3 in development:}

\section{Skin and Hair:}

Gata3 plays an essential role in the stem cell lineage determination in the skin. It is expressed at the onset of inner root sheath (IRS) cell specification in hair follicles. When Gata3 was deleted using a LacZ knock-in, the IRS progenitors failed to differentiate to form IRS, leading to the production of a defective hair structure (Kaufman: 2003)

In addition, Gata3 is the most highly expressed member of the GATA family in the interfollicular epidermis. The specific deletion of Gata3 from the epidermal layer, using a keratin-14-Cre (K14-Cre) mouse line showed to be prenatally lethal due to impairment of the skin selective barrier. Those mice showed defects in skin differentiation, abnormal hair follicle organization and delayed hair growth and maintenance. Genomic analysis of those mice revealed a defective lipid biosynthesis. This could be contributed to the loss of lipid 
acetyletransferase gene (Agpat5), a gene that is a direct target of Gata3 (de Guzman Strong: 2006; Kurek: 2007).

\section{Kidney:}

Gata3 is the only GATA factor that is expressed in the urogenital system prior to E12.5. It is necessary for the normal development of the nephric duct. Using a HoxB7-Cre transgenic line, Grote et al. (2008) have specifically deleted Gata3 from the nephric duct, which resulted in severe abnormalities in the urogenital system, and found that Gata3 is required to prevent ectopic metanephric kidney duct formation and premature cell differentiation. Additionally, they have reported that Gata3 haploinsufficincy resulted in renal dysplasia (Grote: 2008).

Gata3 has also been implicated in clear cell renal cell carcinoma (cc-RCC), the most common subtype of RCC. Cooper et al. (2010) found that when Gata3 expression is downregulated by promoter hypermethylation, it results in decreasing the expression of type III TGF- $\beta$ receptor (T $\beta$ RIII), which is a betaglycan protein with tumour suppressor features (Cooper: 2010).

\section{Mammary gland:}

Gata3 is also crucial for mammary gland development. It is the transcription factor with the highest expression in the mammary epithelium as shown by genome-wide transcript analysis of the terminal end bud and mature duct microenvironment (Kouros-Mehr and Werb: 2006). When Gata3 was specifically deleted from the mammary epithelium at the onset of puberty, using 
the murine mammary tumour virus (MMTV) promoter-Cre recombinase (MMTVCre), the mammary glands failed to develop terminal end buds (TEBs) and resulted in ductal abnormal structures. (Kouros-Mehr: 2006, Asselin-Labat: 2007).

GATA3 mutations have been detected in around $10 \%$ of human breast cancer. While the range of somatic mutations was varied, they clustered mainly in the highly conserved C-terminal second zinc finger (Koboldt: 2012).

Data suggest that GATA3 might act as a tumour suppressor gene. Both in vitro and in vivo studies have indicated a potential tumour suppressor role since the loss of Gata3 results in tumour progression and tumour dissemination in a murine luminal breast cancer model (Kouros-Mehr: 2008).

GATA3 expression inhibits breast cancer growth and pulmonary metastasis by repressing metastasis-associated genes such as ID1/-3, KRTHB1, LY6E and RARRES3 (Dydensborg: 2009), and restoration of GATA3 expression in breast cancer cell lines regulates breast cancer differentiation and supresses its dissemination (Kouros-Mehr: 2008).

Moreover, GATA3 was found to promote the expression of microRNA-29b (miR-29b), which in turn induces differentiation, supresses metastasis and changes the tumour microenvironment (Chou: 2013). In addition, low expression of GATA3 was associated with poor survival rate and a more aggressive disease (Kouros-Mehr: 2006), whereas GATA3-expressing breast cancer patients had a better prognosis, were less likely to relapse, and had a better overall survival rate (Oh: 2006). 


\section{1-6-2-Gata3 in the Haematopoietic System:}

Within the haematopoietic system, Gata3 is expressed in T-cells and thymocytes, and in the NK cell lineage, and plays a crucial role in the regulation of the immune system. It was found to be crucial for the development, maintenance, survival and proliferation of early T-cell progenitors. Ting et al. have shown in chimera studies that ES cells with a deleted Gata3 gene were able to contribute to the reconstitution of B-cell, myelomonocytic and erythroid lineages but not thymocytic or T-cell lineages.

Gata3 is a master regulator of $\mathrm{T}$ helper type 2 cells. It regulates the differentiation of Th2 cells by controlling genes that encode Th2 cytokines interleukin-4 (IL4), IL5, and IL13 (Lee: 2006).

Gata3 levels are carefully controlled throughout thymocyte development as levels that are too high are cytotoxic, and levels that are too low cause developmental failure (Ho: 2009).

GATA3 has also been implicated in T-cell acute lymphoblastic leukaemia (T-ALL). Together, T-cell acute lymphocytic leukaemia 1 (TAL1), RUNX1, and GATA3 form a positive interconnected auto-regulatory loop that directly activates $M Y B$ oncogene, thus, reinforcing and stabilising the oncogenic program that contributes to malignant transformation (Sanda: 2012).

In addition, whole genome sequencing of patients with early T-cell precursor acute lymphoblastic leukaemia (ETP-ALL), an aggressive subtype of 
T-ALL, has revealed GATA3 inactivating lesions disrupting the haematopoietic development (Zhang: 2012).

GATA3 has also been linked to other types of lymphoid malignancies. In a genomic profiling study, a GATA3 SNP genotype has been identified in a subtype of childhood acute lymphoblastic leukaemia (ALL), Philadelphia chromosomepositive ALL (Ph-like ALL) that has been associated with early treatment response, higher risk of relapse and overall poor prognosis (Perez-Andreu: 2013). And in anaplastic large cell lymphoma, the absence of GATA3 protein in addition to the presence of suppressive histone (H3K27) trimethylation of GATA3 suggests an epigenetical involvement of the disease pathogenesis (Joosten: 2013).

Gata3 is not necessary for the development of classic natural killer cells (NK) (Vosshenrich: 2006), but is crucial for a specialised subset of them. It is important for the terminal differentiation of NK cells and their exit from the bone marrow, and is crucial for the maintenance of liver-resident NK cells (Ali: 2016).

Gata3 is also highly expressed in the long term repopulating HSC (LTHSC) population (Zhong: 2005; Kent: 2009; Benveniste: 2009; Hosoya: 2010) and in embryonic HSC precursors (Bertrand: 2005; Swiers: 2013). Using null Gata3 mice, a Gata3 total knockout mouse line, Ku et al. have shown that Gata3 deletion results in the production of lower numbers of adult LT-HSCs, and that a lower number of these Gata3-null LT-HSCs are in cycle (Ku: 2012). This indicates that Gata3 is necessary for maintaining normal numbers of LT-HSCs, and that it regulates their entry into the cell cycle. 
However, by using a conditional Gata3 knockout mouse line, a Vav-Cre mediated deletion, Buza-Vidas et al. have shown that deleting Gata3 expression from HSCs after their emergence from HE in the embryo does not affect the ability of HSCs to expand normally and that their numbers remain unaffected in the BM after birth. Moreover, they reported that Gata3 deletion does not affect the ability of HSCs to self-renew (Buza-Vidas: 2010). It should be noted here that the cells used for this study were BM cells isolated from mice at 1-2 weeks of age as donors, in contrast to $\mathrm{Ku}$ et al study (Ku: 2012), where they used cells obtained from E14.5 fetal liver.

More recently, by also using a conditional Gata3 knockout mouse line, Mx1-Cre mediated, Frelin et al. have demonstrated that Gata3 controls the balance of LT-HSC self-renewal and differentiation by regulating their transition from LT-HSC to Intermediate term HSCs (IT-HSC). The IT-HSCs are important in maintaining blood counts at steady state. They differ from LT-HSCs in that they are able to generate myeloid and erythroid progeny for 12 weeks (Benveniste: 2010), are more abundant than LT-HSCs (three times higher) (Foudi: 2009; Benveniste: 2010), are more proliferative, and exit quiescent state of cell cycle more frequently than LT-HSCs; every10-20 days compared to 50-100 days in LTHSCs (Wilson: 2008; Foudi: 2009). In addition, they have reported that Gata3 relocation from the cytoplasm to the nucleus is associated with LT- HSCs exiting quiescence and long-term reconstitution ability reduction. However, the precise function of Gata3 in adult LT-HSCs requires further dissection.

Gata3 is expressed in the sites of intraembryonic haematopoietic cell generation in the mouse (Sp, P-Sp/AGM) (Manaia: 2000), zebrafish (Neave: 
1995), avian (Leonard: 1993), human (Labastie: 1998) and in Xenopus (Bertwistle: 1996; Turpen: 1997).

In the mouse embryo, Gata3 is expressed at low level at E8.5 in the splanchnic mesoderm (Manaia: 2000). However, by E11.5 Gata3 is expressed at high levels throughout the embryo. At this stage, Gata3 deletion was embryonically lethal, death occurring concomitantly with the onset of definitive haematopoiesis in the fetal liver (Pandolfi: 1995). YS haematopoiesis was normal, which corresponds with the fact the GATA3 is not expressed in the YS (Manaia: 2000).

Gata3 knockout embryos were shown to have growth retardation, along with severe deformities in spinal cord and brain, massive internal haemorrhage, anaemia, and defective liver haematopoiesis, i.e. definitive haematopoiesis, suggesting that Gata3 is essential for the development of various systems (Pandolfi: 1995).

In an in-vitro culture system, the colonies that resulted from the YS of Gata3 knockout embryos, compared to their wild-type and heterozygous littermates were normal, indicating the maintenance of primitive erythropoiesis. However, the colony numbers from the fetal liver of the knockout embryos was low compared to the wild-type and heterozygous littermates, proving that Gata3 disruption severely affects definitive haematopoiesis (Pandolfi: 1995).

Using Ly-6A GFP transgenic embryos, which express GFP in all embryonic HSCs and their precursors, a microarray expression analysis of the AGM region aimed at identifying HSC regulators using a comparative gene expression 
analysis between: (i) HSC containing region (middle part of the dorsal aorta) vs. a region without HSCs (caudal and rostral part); (ii) the microenvironment of HSCs before and after their emergence; i.e. the aorta with the immediate mesenchyme of E9-E10 vs. E11 (iii) HSC-enriched populations and their precursors; intra-aortic clusters, haemogenic endothelium, and pre-HSCs/HSCs (E9 Ly-6A GFP+ cells vs. E11 Ly-6A GFP+ cells). In the microarrays, Gata3 was found to be upregulated in 2 of the 3 comparisons (ii and iii), i.e. in tissues surrounding the dorsal aorta specifically at the time of HSC emergence in the AGM and in pre-HSCs/HSC-enriched populations; E11 Ly-6A GFP+ cells (Mascarenhas: 2009).

Gata3 plays a crucial role in the development of the sympathetic nervous system (Lim: 2000; Moriguchi: 2006; Hong: 2008; Zhao: 2008; Jones and Warchol: 2009). In fact, this is the reason why Gata3 deletion is embryonically lethal. This lethality was attributed to the noradrenaline deficiency in the sympathetic nervous system (SNS) and could be pharmacologically rescued by feeding the mothers DOPS, a synthetic catecholamine intermediate (Lim: 2000).

It was subsequently confirmed that Gata3 is essential for the production of catecholamines, the sympathetic nervous system (SNS) mediators, and plays a major role in the survival of sympathetic neurons in both adults and embryos (Tsarovina: 2004,2010). Interestingly, Gata3 was found to be regulating HSC numbers through catecholamine production, with its absence impairing the production of functional and phenotypic HSCs in the AGM (Fitch: 2012). Previously, it has been reported that the SNS plays a major role in the mobilization (Katayama: 2006), proliferation (Mendez-Ferrer: 2008) and 
repopulation (Spiegel: 2007) of adult HSCs (as discussed above). This indicates the presence of a functional connection between the SNS and haematopoietic system development and that HSC emergence in the AGM should be investigated as a part of a whole developmental process that is influenced by neighbouring tissues (Fitch: 2012).

In addition to its role in the AGM HSC microenvironment described above, Gata3 was also found to be expressed at higher levels in 23GFP+HE than in nonhaemogenic endothelial (NHE) cells (Swiers: 2013), indicating a possible role of its involvement in HSC and progenitor generation. In addition, Gata3 expression increased upon Notch1 signaling induction, which expands the HE population and enhanced their haematopoietic potential (Jang: 2015).

Manaia et al. analysed the expression of Gata3 and Lmo2 in mouse embryonic development to understand the mechanisms involved in the generation of definitive haematopoietic stem cells, with a special emphasis on intraembryonic haemogenic sites (Manaia: 2000). Interestingly, they found that Gata3 and Lmo2 are expressed concomitantly in the caudal embryonic mesoderm where haematopoietic cluster-bearing vessels develop, suggesting an involvement in cell fate determination. Another observation of their study was that Gata3 expression is restricted to sites involved in definitive haematopoiesis. Gata3 is expressed in the environment from which intraembryonic precursors emerge, and in the developing haematopoietic sites before their colonisation. Gata3 was expressed in both thymic rudiment until the first migrants arrived, and inseptum transversum before it gives rise to the FL. However, no CD45+ 
haematopoietic cells were present within those sites at that developmental stage (Manaia: 2000).

Gata3 expression was restricted to the ventral side of the dorsal aorta, where the haematopoietic clusters are found. Its expression was at its highest level at E10.5 (Swiers: 2013), and is physically located below the haematopoietic clusters in the sub-aortic mesenchyme (Fitch: 2012). This expression disappears after E12.5, when intraembryonic clusters cease to be generated. Thus, Gata3 is expressed in three different cell types in the AGM: the SNS, the sub-aortic mesenchyme and the HE, and its involvement in the production of the first HSCs is therefore likely to be complex. The function of Gata3 in these different cell types and how this relates to haematopoietic development was the subject of this $\mathrm{PhD}$ thesis. 


\section{2- Methods}

\section{2-1- Mice and Embryo Generation:}

Timed matings were set up using the following mouse strains: wild-type mice C57BL/6, Gata3-GFP knock in (Grote: 2006), conditional Gata3 knockout (Zhu: 2004), Gata3-LacZ knock in (van Doornick: 1999) and Ve-Cadherin-Cre (Speck: 2009). Embryos were obtained at E9.5, E10.5, and E11.5 where the date of vaginal plug detection is considered as day 0 .

\section{2-2- Genotyping by PCR:}

Embryo heads were cut off, and about $1 / 5^{\text {th }}$ of the head used for genotyping. Genomic DNA was extracted using the HotSHOT method (Truett: 2000). A small part of the embryo head was added to $50 \mathrm{ml}$ of Alkaline lysis buffer $(0.04 \%$ disodium EDTA and $0.25 \% \mathrm{NaOH}$ in water $)$ and heated to $95^{\circ} \mathrm{C}$ at $280 \times g$ shaking for 20 minutes. Then, $50 \mathrm{ml}$ of Neutralisation Reagent was added (4\% 1M Tris- $\mathrm{HCl}$ in water).

The PCR mastermix was comprised of 12.5 ml KAPA2G Fast ReadyMix with dye (Anachem), $2.5 \mathrm{ml}$ of the primer mix (forward and reverse primers), 1 $\mathrm{ml}$ embryo head sample and $9 \mathrm{ml}$ of water per reaction.

Primers and conditions of PCR are detailed in table1. Embryos are then processed based on the experiment that will be carried out. 


\begin{tabular}{|c|c|c|c|c|c|}
\hline Mouse Line & Primer & Sequence & \multicolumn{3}{|c|}{ Program } \\
\hline Gata3 GFP & $\begin{array}{l}\text { ASEQ } 5408 \\
\text { ASEQ } 5409\end{array}$ & $\begin{array}{l}\text { CGCCGCCGGGATCACTCTCG } \\
\text { GATCCAGACATGATAAGATACA }\end{array}$ & $\begin{array}{l}94^{\circ} \mathrm{C} \\
94^{\circ} \mathrm{C} \\
60^{\circ} \mathrm{C} \\
72^{\circ} \mathrm{C} \\
72^{\circ} \mathrm{C}\end{array}$ & $\begin{array}{l}2 \text { minute } \\
30 \text { secon } \\
30 \text { secon } \\
30 \text { secon } \\
30 \text { secon }\end{array}$ & $\begin{array}{l}\text { ] } \\
\text { ] x } 30 \text { cycles } \\
\text { ] }\end{array}$ \\
\hline $\begin{array}{l}\text { Conditional Gata3 } \\
\text { Knock Out }\end{array}$ & $\begin{array}{l}\mathrm{P} 13 \\
\mathrm{P} 16\end{array}$ & $\begin{array}{l}\text { CAGTCTCTGGTATTGATCTGCTTCTT } \\
\text { GTGCAGCAGAGCAGGAAACTCTCAC }\end{array}$ & $\begin{array}{l}94^{\circ} \mathrm{C} \\
94^{\circ} \mathrm{C} \\
55^{\circ} \mathrm{C} \\
72^{\circ} \mathrm{C} \\
72^{\circ} \mathrm{C}\end{array}$ & $\begin{array}{l}5 \text { minute } \\
20 \mathrm{sec}] \\
20 \mathrm{sec}] \\
20 \mathrm{sec}] \\
5 \text { minute }\end{array}$ & x 30 cycles \\
\hline Gata3 LZ & $\begin{array}{l}\text { E2A2 } \\
\text { 913-931 } \\
\text { NLS2 }\end{array}$ & $\begin{array}{l}\text { CGTTGAGGACCGCGGGGTG } \\
\text { CAGGAGTCCGCGGACCTCC } \\
\text { CCAAGCTTGGACTCAAAAAACT }\end{array}$ & $\begin{array}{l}94^{\circ} \mathrm{C} \\
94^{\circ} \mathrm{C} \\
55^{\circ} \mathrm{C} \\
72^{\circ} \mathrm{C} \\
72^{\circ} \mathrm{C}\end{array}$ & $\begin{array}{l}5 \text { minute } \\
30 \mathrm{sec}] \\
30 \mathrm{sec}] \\
30 \mathrm{sec}] \\
10 \text { minut }\end{array}$ & x 35 cycles \\
\hline Vec-Cre & $\begin{array}{l}\text { VECP-F-219 } \\
\text { Cre-R107 }\end{array}$ & $\begin{array}{l}\text { CCCAGGCTGACCAAGCTGAG } \\
\text { GCCTGGCGATCCCTGAACATG }\end{array}$ & $\begin{array}{l}92^{\circ} \mathrm{C} \\
95^{\circ} \mathrm{C} \\
58^{\circ} \mathrm{C} \\
72^{\circ} \mathrm{C} \\
72^{\circ} \mathrm{C}\end{array}$ & $\begin{array}{l}2 \text { minute } \\
15 \mathrm{sec}] \\
15 \mathrm{sec}] \\
5 \mathrm{sec}] \\
10 \mathrm{minut}\end{array}$ & x 30 cycles \\
\hline $\begin{array}{l}\text { Conditional Gata3 - } \\
\text { StellaCre }\end{array}$ & $\begin{array}{l}\text { P8 } \\
\text { P16 }\end{array}$ & $\begin{array}{l}\text { TCAGGGCACTAAGGGTTGTTAACTT } \\
\text { GTGCAGCAGAGCAGGAAACTCTCAC }\end{array}$ & $\begin{array}{l}94^{\circ} \mathrm{C} \\
94^{\circ} \mathrm{C} \\
55^{\circ} \mathrm{C} \\
72^{\circ} \mathrm{C} \\
72^{\circ} \mathrm{C}\end{array}$ & $\begin{array}{l}5 \text { minute } \\
20 \mathrm{sec}] \\
20 \mathrm{sec}] \\
20 \mathrm{sec}] \\
5 \text { minute }\end{array}$ & x 30 cycles \\
\hline
\end{tabular}

Table1: Genotyping primers and PCR conditions

\section{2-3- AGM Dissection:}

On the desired gestation day, the embryos were harvested in sterile complete medium (10\% FCS (Hyclone, Fisher Scientific) and 1\% pen/strep in PBS (Sigma)). All dissections were performed under a Nikon SMZ800 dissection 
microscope (Nikon) while the embryos and tissues were in complete medium. After embryos extraction from the uterine lumen, the developmental stage was specified by either counting somite pairs (E9.5-E10.5) or determining the stages by the eye development (E11.5). Embryos smaller than their littermates or lacking a heartbeat were excluded. The AGM was then dissected, and the planned experiment protocol was followed.

\section{2-4- Flow cytometry and cell sorting:}

\section{2-4-1- AGM:}

AGMs were dissected from the embryos as described above, added to PBS supplemented with 10\% FCS (complete medium) and 1\% PenStrep with $0.125 \%$ collagenase (Alfa Aesar), incubated at $37^{\circ} \mathrm{C}$ for 45 minutes, and mixed by pipetting until the AGMs were fully dissociated. The cell suspension was then washed with $1 \mathrm{ml}$ of complete medium, spun down at $4^{\circ} \mathrm{C}, 250 \times \mathrm{g}$ for 5 minutes, and the supernatant discarded.

Antibody staining on dissociated cells was performed for 30 minutes on ice in the dark. The antibody fluorochrome and concentrations are supplied in table 2

After the staining, the cells were washed with $1 \mathrm{ml}$ FACS buffer (2\% FCS in PBS), spun down at $4^{\circ} \mathrm{C}, 250 \times$ g for 5 minutes, the supernatant discarded, and the cell pellet then resupended in 300 ml FACS buffer containing a dead cell marker. Dead cells were excluded either via 7-aminoactinomycin D staining 
1:1000 (7AAD, Invitrogen) or Sytox 7AAD (1:5000) (Invitrogen). All experiments included the following controls: unstained cells, single stained sample for each flurochrome. When sorting, additional fluorescent minus one (FMO) controls were also used.Cells were analysed using LSRFortessa (BD Bioscience) or sorted using MoFlo (Beckman Coulter), ARIA (BD Bioscience), and Fusion (BD Bioscience).

\begin{tabular}{|l|l|l|l|}
\hline Antibody & Fluorochrome & Concentration & Manufacturer \\
\hline CD34 & PB & $1: 100$ & Biolegend \\
\hline CD34 & PE & $1: 100$ & Biolegend \\
\hline CD31 & APC & $1: 100$ & Biolegend \\
\hline CD41 & BV421 & $1: 100$ & BD Bioscience \\
\hline CD45 & BV421 & $1: 100$ & BD Bioscience \\
\hline CD45 & APC-Cy7 & $1: 100$ & BD Bioscience \\
\hline CD45 & A-700 & $1: 50$ & Biolegend \\
\hline CD43 & BV421 & $1: 100$ & BD Bioscience \\
\hline TER-119 & V500 & $1: 00$ & BD Bioscience \\
\hline PDGFR-b & APC & $1: 200$ & Biolegend \\
\hline PDGFR-b & PE & $1: 100$ & Biolegend \\
\hline Ve-cadherin & PE-Cy7 & $1: 100$ & Biolegend \\
\hline Ve-cadherin & Alexa Fluor 647 & $1: 100$ & BD Bioscience \\
\hline
\end{tabular}

Table 2: Antibodies Fluorochrome and concentration for FACS 


\section{2-4-2- Blood Samples:}

Around $200 \mathrm{ml}$ were collected in EDTA tubes (Sarstedt, UK) from each transplanted mouse. Red Cell Lysis buffer was prepared at RT by diluting the stock solution (BD Biosciences) 1:10 in water. $700 \mathrm{ml}$ of the diluted buffer was added to each blood sample, and incubated in the dark at $4^{\circ} \mathrm{C}$ for 6 minutes. The tubes were then spun down at $4^{\circ} \mathrm{C}, 250 \times \mathrm{g}$ for 5 minutes, the supernatant was discarded, and the pellet resupended in 700 ml FACS buffer (2\% FCS in PBS, Sigma). After that, the samples were filtered through a $50 \mathrm{~mm}$ filter (Partec) into $5 \mathrm{ml}$ round-bottom polystyrene tubes (SLS). Another $700 \mathrm{ml}$ were added to the original tube and filtered to ensure all cells were removed from both the blood tube and the filter. The samples were spun again at the same conditions and resuspended in staining mastermix.

The mastermix was prepared using the following antibodies: CD45.1 (Biolegend) 1:200 on PE, CD45.2 (Biolegend) 1:100 on A-700, CD3 (Biolegend) 1:200 on APC, B220 (Biolegend) 1:200 on APC, MAC1 (Biolegend) 1:200 on PB, and Gr1 (Biolegend) 1:200. Dead cells were excluded via 7-aminoactinomycin D staining 1:1000 (7AAD, Invitrogen). Cells were analysed using LSRFortessa (BD Bioscience)

\section{2-5- Cell Cycle Analysis:}

First, DAPI staining solution was prepared as 5ug/ml DAPI (Sigma) and 1\% (v/v) Nonidet P40 (Sigma) in dH2O. Sorted cells were stained for one 
minute (1:1) at room temperature, and then analysed using an LSRFortessa (BD Bioscience).

\section{2-6- AGM explant culture:}

AGMs from E11.5 and E10.5 embryos were cultured on Durapore filters (Millipore) at the air-liquid interface in M5300 long-term culture medium (Stem Cell Technologies) supplemented with $10^{-6}$ M hydrocortisone (Sigma).

After 3 days, AGMs were dissociated with collagenase as described above and single-cell suspensions were either processed using flow cytometry (see above) or plated in in methylcellulose (see above).

\section{2-7- Colony-Forming Assays:}

Dissociated AGM cells (either fresh or after explant culture) were added to $3.6 \mathrm{ml}$ methylcellulose (M3434: Stem cell technologies) and plated in triplicates $\left(65.000\right.$ cells/plate). Plates were incubated at $37^{\circ} \mathrm{C}$ and haematopoietic colonies were counted and scored 7 days later.

To determine the genotype of colonies when Gata3 was deleted from endothelium, each colony is picked from methylcellulose plate and added to $50 \mathrm{ml}$ of Alkaline lysis buffer $(0.04 \%$ disodium EDTA and $0.25 \% \mathrm{NaOH}$ in water) and heated to $95^{\circ} \mathrm{C}$ at $280 \times \mathrm{g}$ shaking for 20 minutes. Then, $50 \mathrm{ml}$ of Neutralisation Reagent was added (4\% 1M Tris-HCl in water). 
The PCR mastermix was comprised of $12.5 \mu \mathrm{l}$ KAPA2G Fast ReadyMix with dye (Anachem), $2.5 \mu \mathrm{l}$ of the primer mix (forward and reverse primers), and $10 \mathrm{ml}$ colony sample per reaction.

\section{2-8-0P9 cells maintenance:}

OP9 cells were maintained in aMEM (Gibco) with 20\% heat deactivated FCS (Hyclone) and $0.22 \%$ sodium bicarbonate (Gibco) at $37{ }^{\circ} \mathrm{C}$ with $5 \% \mathrm{CO} 2$. Cells were passaged every $2-3$ days using $0.05 \%$ Trypsin/EDTA (Gibco) at $37{ }^{\circ} \mathrm{C}$. For co-culture experiments, cells were resuspended in aMEM with 10\% FCS and $0.01 \%$ of 2 -mercaptoethanol and plated 24 hours before the experiment.

\section{2-9- OP9 Co-cultures:}

OP9 co cultures and maintenance were performed using the protocol supplied by Swiers et al. Briefly, cells were sorted and plated on confluent OP9

stroma. For haemogenic assays, cultures were supplemented with SCF, FLt-3 ligand and IL7 at 10ng/ml concentration (all from Pepro-Tech) and incubated for 8-10 days at $37^{\circ} \mathrm{C}, 5 \% \mathrm{CO}$. The haematopoietic nature of the cells generated in the OP9 co cultures was assessed by CFU-C and flow cytometry.

\section{2-10- OP9 Co-Aggregates:}

Cells from E11.5 or E10.5 embryos were sorted and co-aggregated with OP9 cells according to the protocol supplied by Rybtsov et al (2001). 
Cell suspensions containing 1 embryo equivalent (e.e) of the sorted cells and $10^{5}$ OP9 cells in $30 \mathrm{ml}$ volume of media (Iscove's modified Dulbecco's medium [IMDM], Invitrogen-GIBCO, 20\% of heat-inactivated FCS, L-glutamine, penicillin/streptomycin) were centrifuged at $450 \mathrm{x}$ g for $12 \mathrm{~min}$ in $200 \mathrm{ml}$ pipette tips sealed with parafilm (Taoudi et al., 2008). The IMDM media for the culture steps was supplemented with SCF, Flt3 and IL3 at a concentration of 100 ng/ml (all from Pepro-Tech). Coaggregates were cultured on floating $0.8 \mathrm{~mm}$ AAWP $25 \mathrm{~mm}$ nitrocellulose membranes (Millipore) for 4-5 days. Cultured aggregates were dissociated using collagenase and either processed using flow cytometry (see above), or plated in methylcellulose (see above), or transplanted into irradiated mouse recipients.

\section{2-11- Transplantation Experiments:}

The dissociated cell suspensions were spun down at $4^{\circ} \mathrm{C}, 250 \times \mathrm{g}$ for 5 minutes and then resuspended in $500 \mathrm{ml}$ PBS. The cell suspensions were injected intravenously into irradiated recipients together with $2 \times 10^{5}$ spleen cells or $2 \mathrm{x}$ $10^{4} \mathrm{BM}$ cells to alleviate the irradiation effects. The irradiation it self was split into two doses, each dose being 460-475 rad with three hours between each dose. The congenic CD45 system was used with allelic variants of CD45 (CD45.1 and CD45.2) to track the donor contribution to the recipient haematopoietic cells. Recipients were CD45.1/.2 or CD45.1/.1 and the donors were CD45.2/.2 on a C57BL6 background. The helper cells (spleen or BM) were either CD45.1/.2 or CD45.1/.1, to avoid contribution to the donor percentage. Recipient blood was 
analysed at 1 and 4 months after the transplantation. The recipients were considered positive if the donor contribution was more than $5 \%$.

\section{2-12- Tissue preparation for Immunohistochemistry:}

Tissues and embryos were fixed in 2\% paraformaldehyde (PFA, Sigma) and phosphate buffered saline (PBS; Cambrex or Sigma) for 1.5 hour at $4^{\circ} \mathrm{C}$, while rotating, then washed three times in PBS, and cryoprotected overnight in $30 \%$ sucrose $/ \mathrm{PBS}$ at $4^{\circ} \mathrm{C}$.

Fixed tissues were then embedded in OCT TissueTek Compound and quick-frozen on dry ice, before being transferred to $-80^{\circ} \mathrm{C} .10 \mathrm{~mm}$ sections were generated on a cryostat (Leica, CM3050 S).

\section{2-13- Immunohistochemistry (IHC):}

For staining, either an Avidin/biotin system or fluorescent-labelled secondary antibodies were used. PBS with 1\% FCS and $0.1 \%$ Triton was used for washing between steps. For blocking, PBS with $0.05 \%$ Tween and 1\% BSA was used ('blocking solution'). Slides were allowed to dry at room temperature after being taken out from $-80^{\circ} \mathrm{C}$ for 10 minutes. They were then washed three times with PBS for five minutes, blocked with $200 \mathrm{ml}$ of the blocking solution or with the Avidin/biotin complex. The primary antibody was then added, and the slides were incubated for 24 hours at $4^{\circ} \mathrm{C}$ in a dark humidified box. After the incubation, the slides were then washed with PBS and the secondary antibody 
was added, incubated for 45 minutes at room temperature in a dark humidified box. The slides were then washed with PBS, mounted with Vectashield with DAPI (Vectorlabs), sealed with nail varnish and placed in a dark box until the time of analysis. Antibody concentrations and providers are detailed in Table 2.

\begin{tabular}{|l|l|}
\hline Antibody & Concentration \\
\hline TH on Mouse (Millipore) & $1: 300$ \\
\hline GFP on Rabbit (Lifetechnologies) & $1: 500$ \\
\hline P75 on Goat (Santa Cruz) & $1: 100$ \\
\hline CD34 on Rat (BD-Pharmingen) & $1: 100$ \\
\hline Anti rabbit Alexa 555 (Invitrogen) & $1: 500$ \\
\hline Anti mouse Alexa 488 (Invitrogen) & $1: 500$ \\
\hline Biotin-conjugated anti-rabbit antibody (DAK0) & $1: 100$ \\
\hline Streptavidin-Cy5 (Jackson Immunoresearch) & $1: 100$ \\
\hline PDGFRb on Rabbit (CellSignal) & $1: 100$ \\
\hline Anti mouse Alexa 546 (LifeTechnologies) & $1: 200$ \\
\hline Anti-donkey 488 Alexa (LifeTechnologies) & $1: 200$ \\
\hline GFP on Chicken (LifeTechnologies) & $1: 400$ \\
\hline Anti chicken 647 Alexa (Millipore) & $1: 200$ \\
\hline Anti Rabbit Alexa 647 (Lifetechnologies) & $1: 200$ \\
\hline Anti goat 633 (Sigma) & $1: 200$ \\
\hline Runx1 on Rabbit (Abcam) & $1: 100$ \\
\hline
\end{tabular}

Table 3: IHC Antibodies and concentration

\section{2-14- Statistical Analysis:}

All graphs and statistical analysis were performed on GraphPad Prism.

The Mann-Whitney test was used to determine significance levels for 
transplantation experiments, paired t-test was used to determine the significance levels for colony forming assay following OP9 co-culture and coaggregates, and two way ANOVA test was used to determine significance levels for colony forming assay.

\section{2-15-RNA Sequencing:}

The protocol was provided by Nicola Wilson from Berthold Gottgens group (Picelli: 2014). All steps were done in a UV-sterilised hood with laminar flow. To ensure that all surfaces were free of RNase and DNA, all surfaces were treated with DNase/RNase Zap.

\section{2-15-1-Cell lysis:}

Cells were sorted into eppendorf tubes based on their populations: G3+EC, G3-EC, G3+HC, G3-HC, G3+MC, G3-MC, G3+SNS and G3-SNS. Each population was then sorted again into 96 wells plate, 20 cells/well, containing $2.3 \mu \mathrm{l}$ of lysis buffer comprised of $0.2 \%$ RNase inhibitor (Ambion, Thermo Fisher Scientific) in Triton X-100 (Sigma) in each well. Plates were spun down and stored at $-80^{\circ} \mathrm{C}$ until processed.

Cells were collected from 3 different experiments for MC and SNS, and 4 different experiments for EC and HC. 


\section{2-15-2-Reverse Transcription:}

The annealing mixture was made up of 5\% ERCC RNA spike-In Mix (prediluted at 1:25,000; Invitrogen, Thermo Fisher Scientific), 5\% Oligo-dT (5'AAGCAGTGGTATCAACGCAGAGTACT30VN-3'; 100 mM; biomers.net), 50\% dNTP 10mM (Fermentas, Thermo Fisher Scientific) and 40\% distilled water. Then, $2 \mu \mathrm{l}$ of annealing mix were added to each well, and the plate was centrifuged at $700 \times$ $\mathrm{g}$ for 1 minute. The samples were then incubated a $72^{\circ} \mathrm{C}$ for 3 minutes and immediately placed on ice, and then centrifuged again at $700 \mathrm{xg}$ for 1 minute.

The reverse transcription mixture was made up of: $0.5 \mu \mathrm{l} \quad(8.7 \%)$ Superscript II RT (200 U/ $\mu \mathrm{l}$; Invitrogen, Thermo Fisher Scientific), $0.25 \mu \mathrm{l}$ (4.3\%) RNase inhibitor (20 U/ml), $2 \mu \mathrm{l}$ (35\%) 5x Superscript II First Strand Buffer (Invitrogen, Thermo Fisher Scientific), $0.5 \mu \mathrm{l}$ (8.7\%) DTT (Invitrogen, Thermo Fisher Scientific), $2 \mu \mathrm{l}$ (35\%) 100 mM Betaine (Sigma), $0.06 \mu \mathrm{l}$ (1\%) 1 M MgCl2 (Ambion, Thermo Fisher Scientific), $0.1 \mu \mathrm{l}$ (1.7\%) TSO Oligo (5'AAGCAGTGGTATCAACGCAGAGTACATrGrG+G-3'; $100 \mathrm{mM}$; Exiqon) and $0.29 \mu \mathrm{l}$ (5\%) distilled water per well. $5.7 \mu \mathrm{l}$ of the reverse transcription mixture were added per well, and the plate was centrifuged at $700 \times \mathrm{g}$ for 1 minute. The plate was then placed in a PCR cycler with the following settings: $42^{\circ} \mathrm{C}$ for 90 minutes, 10 cycles of: $50^{\circ} \mathrm{C}$ for 2 minutes, $42^{\circ} \mathrm{C}$ for 2 minutes, then at the end $70^{\circ} \mathrm{C}$ for 15 minutes. 


\section{2-15-3- PCR Pre-Amplification:}

For the PCR Amplification, a PCR mixture was produced out of $12.5 \mu \mathrm{l}$ (83\%) KAPA HiFi Hotstart ReadyMix (2x; KAPA Biosystems), $0.25 \mu$ l (2\%) ISPCR primer (5'-AAGCAGTGGTATCAACGCAGAGT-3'; $10 \mathrm{mM}$; biomers.net) and $2.25 \mu \mathrm{l}$ (15\%) distilled water per well. $15 \mu \mathrm{l}$ of the PCR mixture were added per well, and the plate was centrifuged at $700 \times \mathrm{g}$ for 1 minute. The plate was placed in a PCR cycler after that with the following settings: $98^{\circ} \mathrm{C}$ for 3 minutes, 21 cycles of: $98^{\circ} \mathrm{C}$ for 20 seconds, $67^{\circ} \mathrm{C}$ for 15 seconds, $72^{\circ} \mathrm{C}$ for 6 minutes, and then at the end $72^{\circ} \mathrm{C}$ for 5 minutes.

Ampure XP beads (Beckman Coulter) were used for PCR clean up. It was equilibrated at room temperature (RT) for 15 minutes and then vortexed for several seconds. These beads were then added to each sample at a 1:1 ratio and mixed by pipetting until the solution appeared homogeneous. The sample/bead mixture was left to incubate at RT for 8 minutes before fitting the 96-well plate on a plate magnetic stand. The plate was left on the stand for 5 minutes until all beads had been collected at one well corner. The supernatant was discarded, and the beads were washed twice with $200 \mu$ of $80 \%$ ethanol after incubating the ethanol/bead mixture for 30 seconds each time. The last ethanol traces were removed with yellow tips, leaving the beads to dry completely (approximately 37 minutes), indicated by a 'cracked' appearance. Then, $20 \mu \mathrm{l}$ of Elution Buffer (Qiagen) were added and mixed with the beads by pipetting. The plate was incubated off the magnet for 2 minutes and then placed back on the magnetic stand for 2 minutes. Finally, the supernatant was collected without disturbing the beads and transferred to a new 96-well plate. 


\section{2-15-4- Quality Check of the cDNA Library:}

The size distribution of the cDNA library was checked on an Agilent highsensitivity DNA chip (Agilent Technologies), according to the manufacturer instructions. This was done for 11 random wells of each plate. Briefly, the gel-dye mix (composed of 'High Sensitivity DNA dye concentrate' and 'High Sensitivity DNA gel matrix') was allowed to equilibrate to RT for 30 minutes. A new 'High Sensitivity DNA chip' was placed on the chip priming station and $9 \mu$ of gel-dye mix were pipetted into the priming station and distributed across the chip using

the plunger. Additional $9 \mu \mathrm{l}$ per well were added to the other control wells. $5 \mu \mathrm{l}$ of a marker was added to each well in addition to $1 \mu \mathrm{l}$ of 'High Sensitivity DNA ladder' or sample, depending on the well position on the chip. The chip was then vortexed for 1 minute at $2400 \mathrm{rpm}$ on an IKA vortexer and loaded on the Agilent 2100 Bioanalyser instrument (Agilent Technologies).

\section{2-15-5- Library Preparation:}

To break up the DNA strands, transposon mediated fragmentation (tagmentation) was carried out using the Illumina Nextera XT DNA sample preparation kit (Illumina) according to an optimised Tagmentation protocol (Fluidigm). NT buffer and Tagment DNA Buffer were equilibrated to RT and vortexed until all precipitated particles were resuspended. Per cell, a pre-mix was made, the volume per sample consisted of: $2.5 \mu$ tagmentation DNA buffer, $1.25 \mu \mathrm{l}$ Amplification Tagment mix and $1.25 \mu \mathrm{l}$ of the eluted cDNA, and for each cell a different well of a 96-well plate was used. The plate was sealed and centrifuged at 4,000 rpm for 5 minutes, before being placed in a thermal cycler 
for 10 minutes at $55^{\circ} \mathrm{C}$. Then, the NT buffer was added at $20 \%$ ratio to the original volume in each well and the plate was sealed and centrifuged again at 4,000 rpm for 5 minutes. After that, Nextera PCR Master Mix (NMP) was added at a ratio of $37.5 \%$ to the initial volume and Index Primers 1 (N701-N712) and 2 (S501-S508) at a ratio of $12.5 \%$ (each) were combined in a way that each well was uniquely labelled and dual-indexing metadata could be obtained (Nextera XT 96-Index kit; Illumina). The plate was centrifuged again at $4000 \mathrm{rpm}$ for2 minutes, and PCR amplification was performed in a thermal cycler at these settings: $72^{\circ} \mathrm{C}$ for 3 minutes, $95^{\circ} \mathrm{C}$ for 30 seconds, 12 cycles of: $95^{\circ} \mathrm{C}$ for 10 seconds, $55^{\circ} \mathrm{C}$ for 30 seconds, $72^{\circ} \mathrm{C}$ for 60 seconds and then at the end $72^{\circ} \mathrm{C}$ for 5 minutes.

\section{2-15-6- Library Pooling and Clean-up:}

Ampure XP beads were equilibrated at RT for 15 minutes and then vortexed for several seconds. One library was made per plate by pooling $1 \mu \mathrm{l}$ of each well and adding beads at $70 \%$ of the total pool volume, mixing by pipetting. The tube containing the library mix was placed on a magnetic stand and the supernatant was removed. Two washing steps were performed by adding $1.2 \mathrm{ml}$ of freshly prepared $80 \%$ ethanol and incubating the mixture for 30 seconds on the magnetic stand. The beads were allowed to air-dry for 5-10 minutes, then the sample was eluted in $50 \mu \mathrm{l}$ of elution buffer. 


\section{2-15-7- Library Quality Check:}

The library size distribution was checked on an Agilent high-sensitivity DNA chip as described above.

\section{2-15-8- Library Quantification:}

The pooled library was quantified using the KAPA library quantification kit (KAPA Biosystems). For each reaction, 60\% (6 $\mu \mathrm{l}$ ) of '2X KAPA SYBR FAST qPCR Master Mix +10X Primer Premix' was mixed with 20\% (2 $\mu$ l) PCR-grade water and 20\% (2 $\mu \mathrm{l})$ of diluted library solution. Each library was diluted in DNA Dilution Buffer (10 mM Tris-HCl, pH 8.0 and 0.05\% Tween 20) at 1:2,000,000 and run in triplicates along with the provided DNA Standards (Standards 3-6 were used) and No Template Controls. The following settings were used: $95^{\circ} \mathrm{C}$ for 5 minutes, 35 cycles of: $95^{\circ} \mathrm{C}$ for 30 seconds, $60^{\circ} \mathrm{C}$ for 45 seconds and $65-95^{\circ} \mathrm{C}$ for a melting curve step. For the data analysis, a standard curve was generated and the concentration was calculated for each library.

\section{2-15-9- Sequencing \& Data Analysis:}

Pooled libraries were sequenced on an Illumina Hi-Seq 2500 (Sanger, Cambridge), as single-end 125 base pair reads. The samples were run on two lanes of a flowcell of 8 lanes. Each lane had 4 populations that were pooled together. This was achieved in collaboration with Nicola Wilson. Lila Diamanti from the WT-MRC Cambridge Stem Cell Institute performed the data analysis. 
The threshold for the quality control of the data were:

1- More than 500,000 reads mapped (either to ERCC spike-ins or endogenous mRNA)

2- More than $20 \%$ of total reads mapped to mRNA

3- Less than $20 \%$ of mapped reads allocated to mitochondrial genes

4- Less than $20 \%$ of reads mapped to ERCC spike-ins

5- More than 8000 high coverage genes

Gene Ontology (GO) analysis was done using the Gene Ontology Consortium Enrichment Analysis, which utilise PANTHER Classification System for biological processes in mus musculus. 


\section{3- Gata3 expression marks haemogenic endothelium}

\section{3-1- Background:}

Previously, the Ottersbach group carried out microarray expression analyses of the AGM region to identify HSCs regulators. Among the genes that were upregulated at the time of HSCs emergence was Gata3 (Mascarenhas: 2009).

Subsequently, the Ottersbach group has identified that GATA3 regulates HSCs generation indirectly by the means of the SNS. Gata3-deficient embryos could not develop a functional SNS, and as a result could not produce catecholamines the SNS mediators, which caused a marked reduction in both HSCs and haematopoietic progenitors in these embryos (Fitch: 2012).

However, a more direct role of Gata3 in haematopoiesis is yet to be investigated. Gata3 was previously reported to be expressed in endothelial cells, mesenchymal cells and sympathoadrenal cells within the AGM region (Fitch 2012; Manaia: 2000) and has subsequently been shown to be enriched in haemogenic endothelial cells (Swiers: 2013), where it was reported to enhance the haematopoietic potential of those cells (Jang: 2015).

Given the correspondence of both temporal and spatial expression of Gata3 with the activity of HE and the generation of HSCs and progenitor cells, we therefore hypothesised that Gata3 could potentially mark haemogenic endothelium and have a distinct and more direct role in HSC and progenitor formation.

To enable us to further investigate and isolate Gata3-expressing cells, we used a G3-GFP reporter mouse (G3-GFP) line (Grote: 2005) in order to isolate those Gata3-expressing cells and to characterise them. 


\section{3-2-Aims:}

- $\quad$ Characterise Gata3-expressing cells using the G3-GFP reporter mouse line

Investigate the haematopoietic potential of Gata3-expressing endothelial and haematopoietic cells.

Determining the type and place of G3+ HC in the haematopoietic differentiation pathway 


\section{3-3-GATA-3 is expressed in various cell types in the E10.5 and E11.5}

AGM:

\section{3-3-1 E11.5:}

Initially, we wanted to confirm if the GFP in our mouse line was a faithful reporter to the indigenous expression of Gata3. For that reason, I stained a section from an E11.5 G3-GFP+ embryo and used both an anti-Gata3 antibody along with an anti-GFP antibody. The staining showed that indeed Gata3 and GFP stained the same cells. However, the staining patterns were different in those cells, as Gata3 is intranuclear while GFP is extranuclear in the cytoplasm (Figure 3-1).

In flow cytometry analysis, only cells from embryos that were G3-GFP+ have shown a definitive spike in the G3-GFP region, which was confirmed by RTPCR (Figure 3-2 and data not shown).
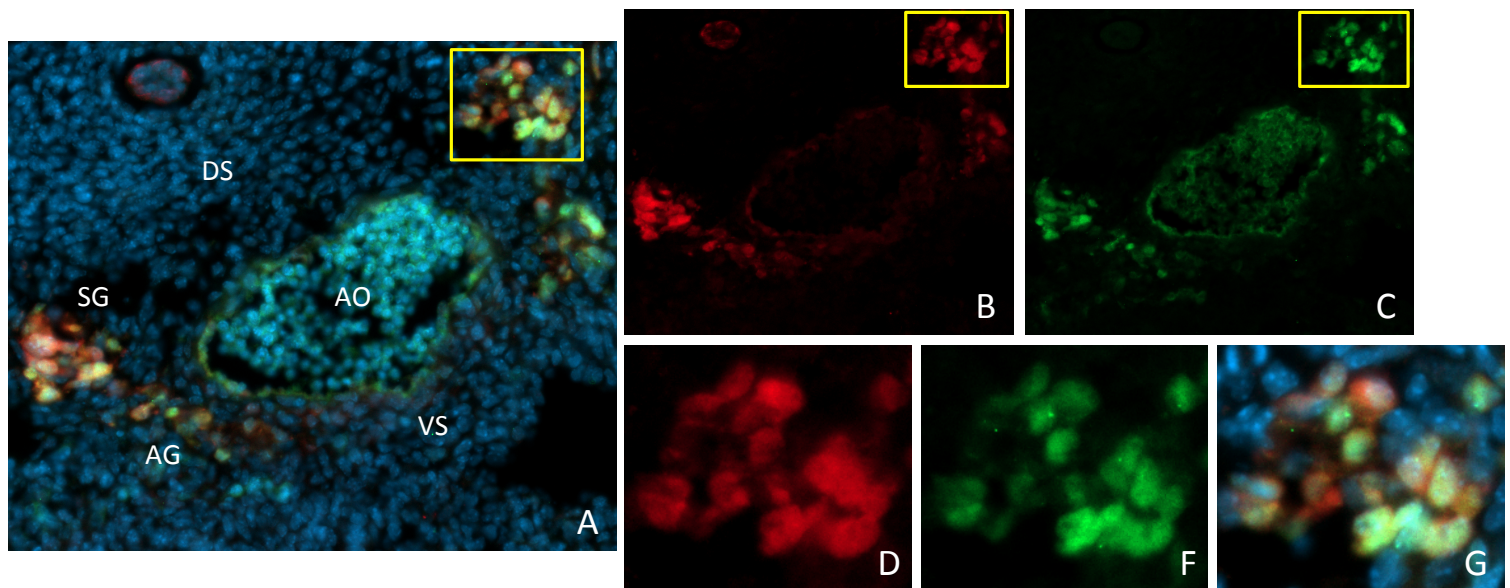

Figure 3-1: A section from a G3-GFP+ E11.5 embryo showing the co-staining between Gata3 (green) and GFP (red). (A) merged image, (B) antibody to GFP, (C) antibody to Gata3. (D-G) Magnification of cells indicated by yellow box in image showing the costaining between Gata3 and GFP. Ao: dorsal aorta, AG: adrenal anlage, SG: sympathetic ganglia, DS: dorsal side, VS: ventral side. 


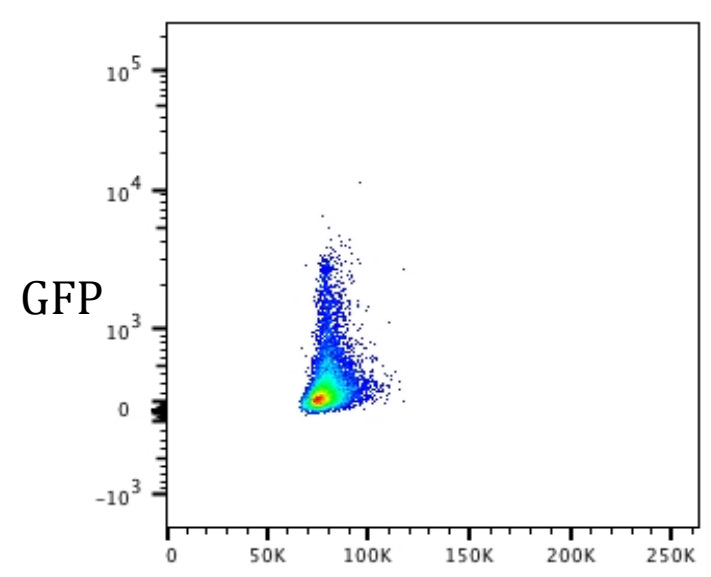

G3-GFP

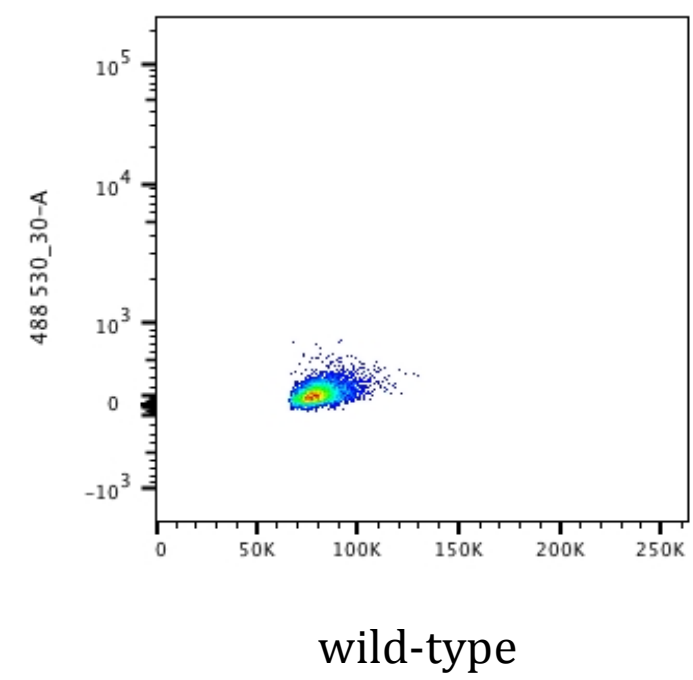

Figure 3-2: FACS analysis showing a definitive spike in the GFP region in the AGM obtained from a G3-GFP E11.5 embryo compared to unstained AGM sample obtained from a wild-type littermate.

Using these markers, several populations that were G3-GFP+ were identified. It should be noted that all G3-GFP+ cells at this stage are CD45(Figure 3-3). G3-GFP+ cells were found both in the positive and negative CD34, PDGFRb, and P75 subfractions. Most of the GFP+ cells at this stage are SNS which compromise $15 \%$ of the total GFP+ cells, followed by mesenchymal cells $(2.6 \%)$, and lastly endothelial cells (1\%). The rest of the GFP+ cells are more likely to belong to the mesonephros duct.

G3-GFP+CD34+ cells were designated as G3+ECs, G3-GFP+PDGFRb+ as mesenchymal cells (G3+MC), G3-GFP+P75+ as SNS cells (G3+SNS), G3-GFPCD34+ as G3-EC, and G3-GFP-PDGFRb- as G3-MC. 


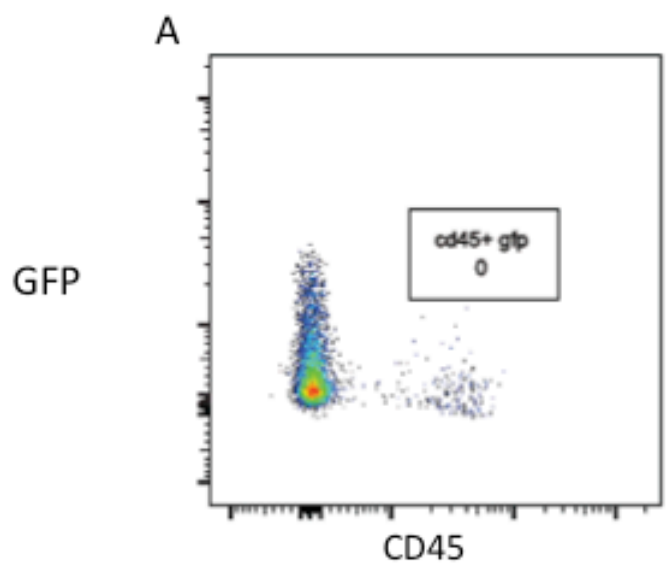

Haematopoietic

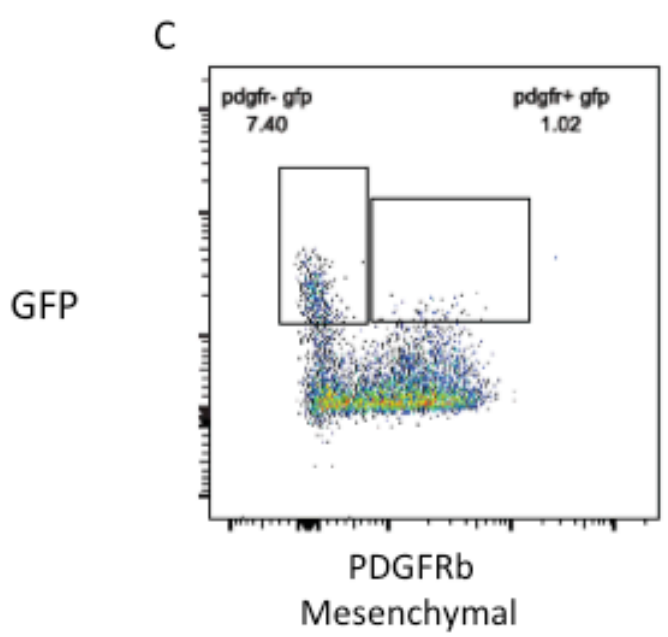

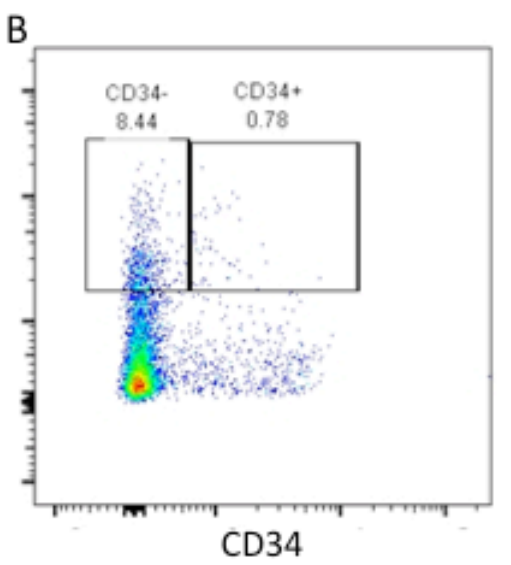

Endothelial

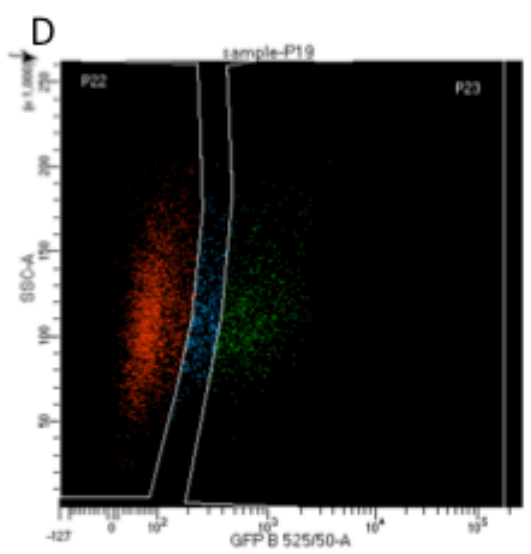

P75 pregated on G3-GFP+ SNS

Figure 3-3: Flow cytometry analysis of G3-GFP+ samples from E11.5 AGMs, stained for CD45, CD34, PDGFRb and P75. No Gata3-GFP was expressed in the HC (A). Three populations were expressing Gata3-GFP: (B) EC, (C) MC, and (D) SNS.

To characterise the spatial distribution of G3-GFP+ cells within the AGM region, embryo sections of G3-GFP+ E11.5 embryos were stained with an antiGFP antibody in combination with antibodies to tyrosine hydroxylase (Th), a rate limiting enzyme for catecholamine synthesis that is expressed in sympathoadrenal cells; CD34, a cell surface marker for endothelial cells in order to identify the location of HECs within the wall of the aorta; Runx1, a transcription factor expressed in haemogenic endothelium cells; P75, a cell surface marker for SNS cells; and PDGFRb, a marker for mesenchymal cells. Here we wanted determine the expression and overlap between Gata3-GFP and the 
different cell populations of interest; Th, CD34, and PDGFRb, P75 and Runx1 in more detail (Figures 3-4, 3-5, 3-6, 3-7, 3-8).

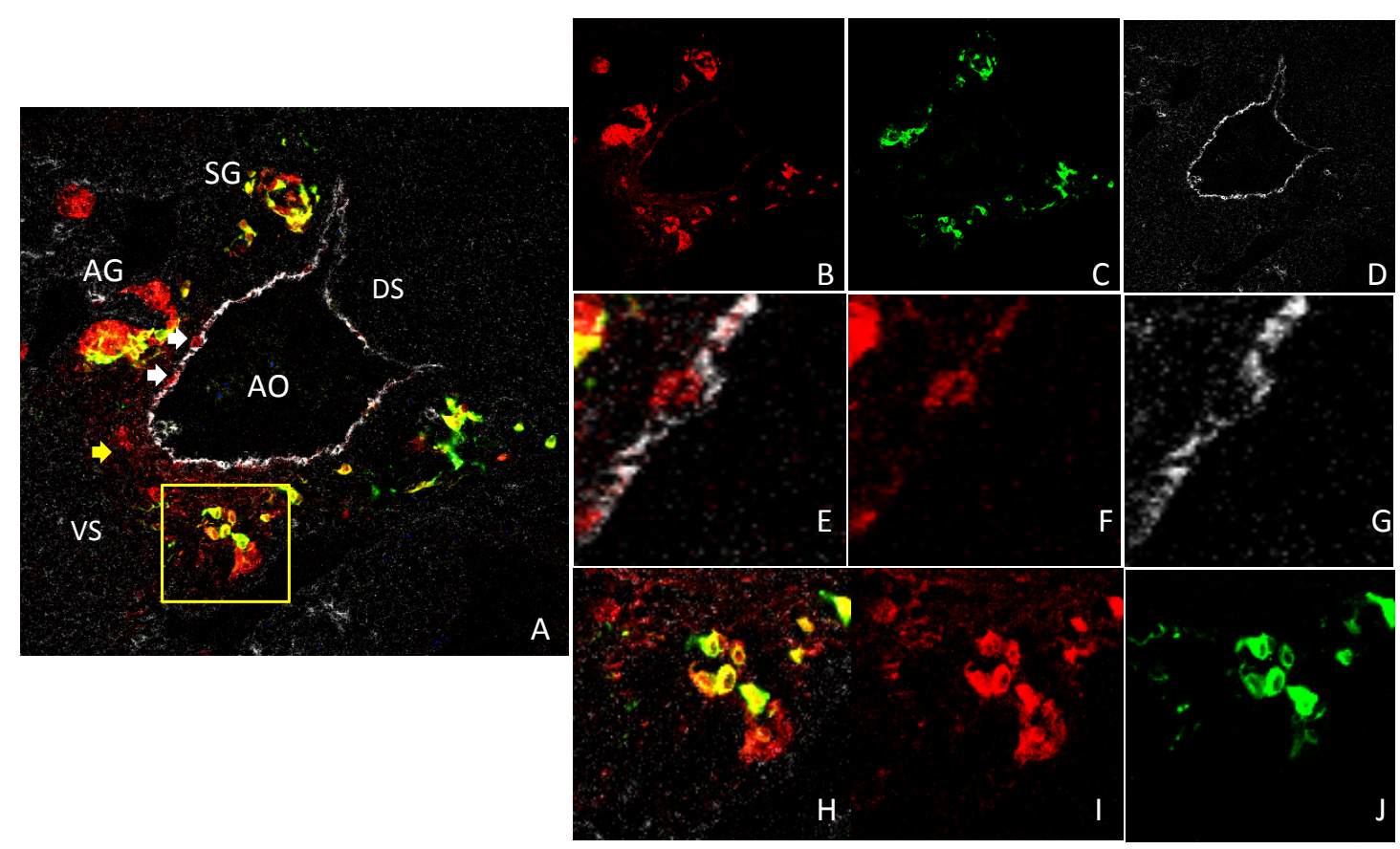

Figure 3-4: (A) section from a Gata3-GFP+ E11.5 embryo stained with (B) Gata3GFP (red), (C) Th (green) and (D) CD34 (white). White arrows shows the costaining between Gata3 and CD34. (E-G) Magnification of cells indicated by white arrow in image (A) shows the co-staining between CD34 (G) and Gata3 (F). (H-I) Magnification of cells indicated by yellow box in image (A) shows the co-staining between Gata3 (I) and Th (J).Yellow arrow highlight the staining of Gata3 in subaortic mesenchyme. . Ao: dorsal aorta, AG: adrenal anlage, SG: sympathetic ganglia, DS: dorsal side, VS: ventral side

The endothelial cells in the walls of the dorsal aorta have been established as the source of progenitors and the first definitive HSCs. Gata3 is expressed in some of those cells (Figure 3-4 A, E-G). Gata3 regulates HSC numbers through catecholamine production and is thus also expressed in SNS cells as previously observed (Fitch: 2012 Tsarovina:2004 ,Moriguchi:2006 ) (Figure 3-4 A, H-J). Gata3 is also expressed in some cells of the mesenchyme ventral to the dorsal aorta, i.e. the sub-aortic mesenchyme region, highlighted by the yellow arrow (Figure 3-4 A). 
Figure (3-4 A) demonstrates an obvious overlap between Th and Gata3 in the adrenal anlage region, where the neural crest cells differentiate to form the SNS. However, only few cells were expressing both Gata3 and CD34 among the CD34+ cells that form the endothelial lining of the dorsal aorta, where HSCs emerge (Figure 3-4 A, E-G).

More stainings were performed to determine the expression and overlap between G3-GFP and the different cell populations of interest; Th, CD34, PDGFRb, P75 and Runx1 in more detail.

To look at the supportive microenvironment of the HSCs (specifically the sub-aortic mesenchyme), sections were obtained from E11.5 G3-GFP+ embryos and stained for PDGFRb, CD34, Th and GFP. The staining showed a subpopulation of the mesenchymal cells, PDGFRb+ cells, that co-expressed Gata3. These cells, G3-GFP+PDGFRb+ were CD34- (Figure 3-4 B) and Th- (Figure 3-5). PDGFRb, and Th showed to be mutually exclusive, with Gata3 being expressed within a subpopulation of each (Figure 3-4, 3-5, 3-6).
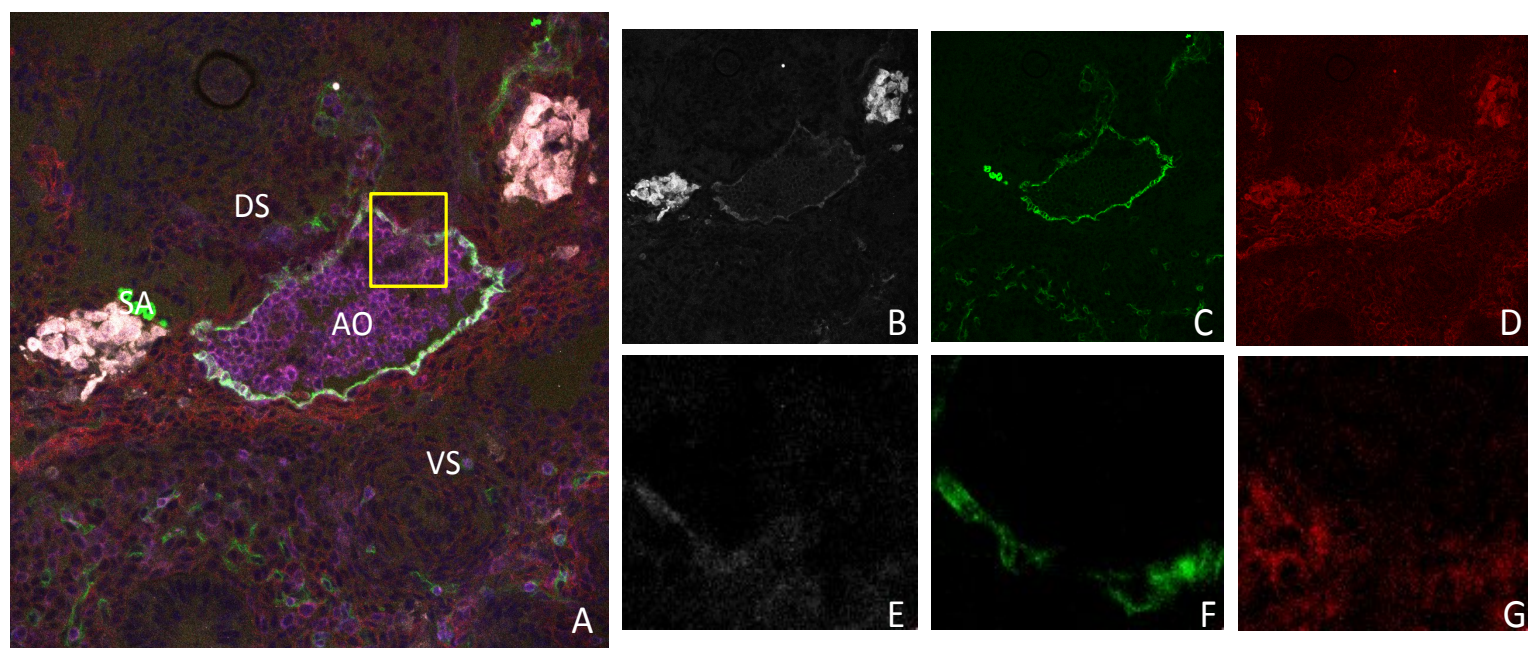

Figure 3-5: (A) section from a Gata3-GFP+ E11.5 embryo stained with $(B, F)$ Gata3GFP (white), (C,G) PDGFRb (red) and (D,H) CD34 (green). (E-H) Magnification of cells indicated by yellow box in image (A) shows the co-staining between Gata3 and CD34, Gata3 and PDGFRb. There was no co-staining between CD34 and PDGFRb. Ao: dorsal aorta, AG: adrenal anlage, SG: sympathetic ganglia, , DS: dorsal side, VS: ventral side. 


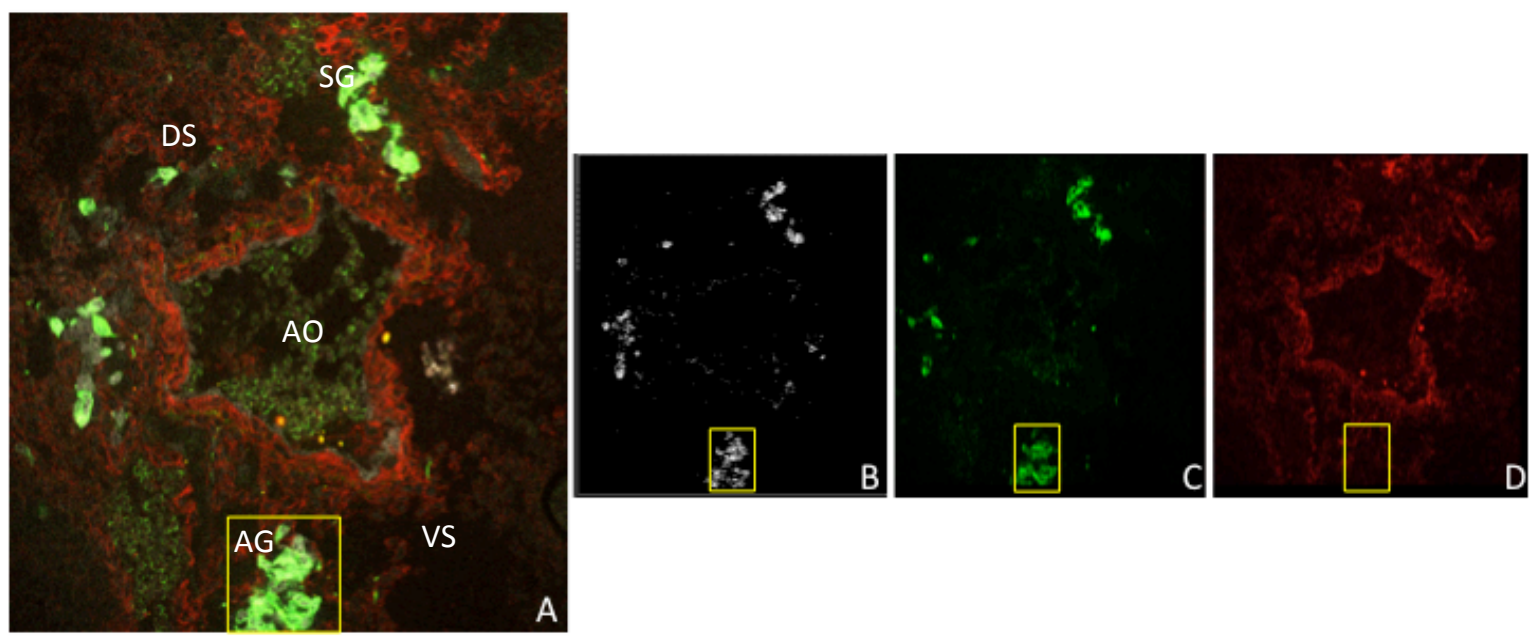

Figure 3-6: (A) section from a Gata3-GFP+ E11.5 embryo stained with (B) Gata3GFP (white), (C) Th (green), and (D) PDGFRb (red). Yellow box shows co-staining between Gata3 and Th, Gata3, but there was no co-staining between PDGFRb and Th. Ao: dorsal aorta, AG: adrenal anlage, SG: sympathetic ganglia, DS: dorsal side, VS: ventral side.

To further investigate the co-staining between G3-GFP and Th-expressing cells, especially since the overlap does not seem to be complete and may represent different stages in the maturation of neural crest cells to SNS cells, a marker for neural crest cells, P75 (Ngfr), was used (Stemple and Anderson: 1992). Neural crest cells, the precursors of sympathoadrenal neurons, migrate from the neural tube to the aorta, where they differentiate into the sympathoadrenal cells. They continue to express P75 throughout this entire process (Stemple and Anderson: 1992).

The IHC stains confirm that P75 expression persists through the migration of these cells and is still expressed in the more differentiated cells of the SNS, where it co-stains with both G3-GFP and Th (Figure 3-7). All the SNS cells (Th+ cells), including G3-GFP+ cells within the SNS, express p75. Thus, P75, a membrane protein, can be used as a marker for SNS cells of the AGM, which makes it possible to isolate this cell population by flow sorting. 


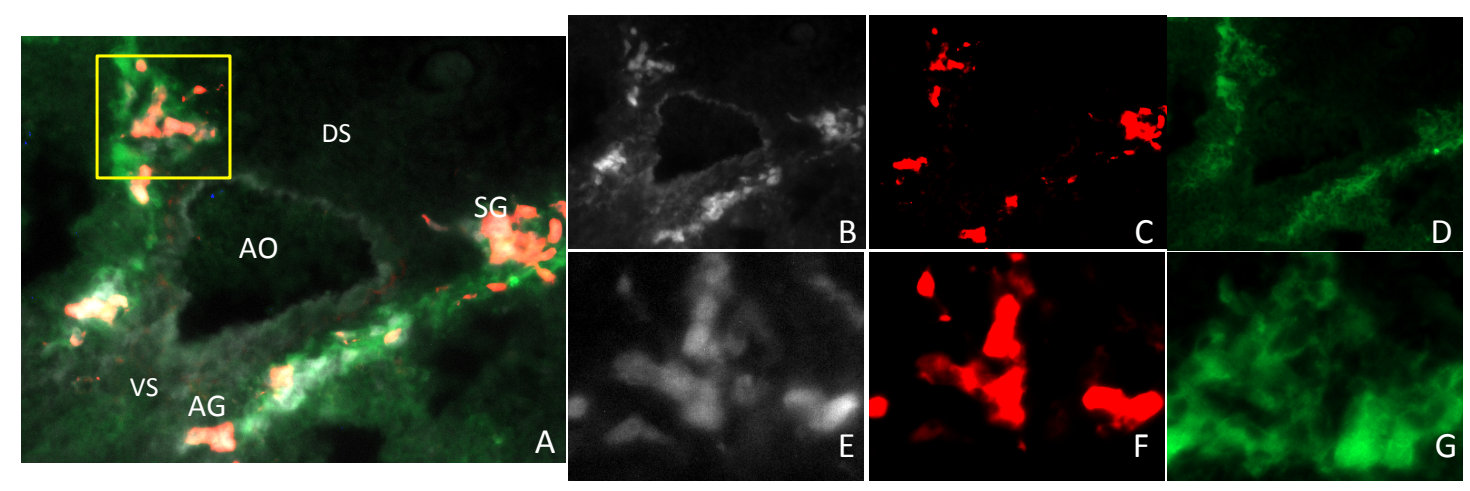

Figure 3-7: (A) section from a Gata3-GFP+ E11.5 embryo stained with $(B, E)$ Gata3GFP (white), (C,F) Th (red), and (D,G) P75 (green). The yellow box highlights one of the areas where there is co-staining between all three markers. (E-G) Magnification of cells indicated by yellow box in image (A). All Th expressing cells are Gata3+ and P75+. Ao: dorsal aorta, AG: adrenal anlage, SG: sympathetic ganglia, DS: dorsal side, VS: ventral side.

Then, we wanted to confirm if Gata3 is co-expressed with Runx1, a known marker for haemogenic endothelium cells and haematopoietic clusters. CD34 was also included to mark the endothelium (Figure 3-8). Gata3, CD34 and Runx1 were co-expressed in some cells, in particular at the ventral side of the dorsal aorta, marked with a yellow box. In addition to that, both Runx1 and Gata3 costained some cells in the subaortic mesenchyme that were CD34-.
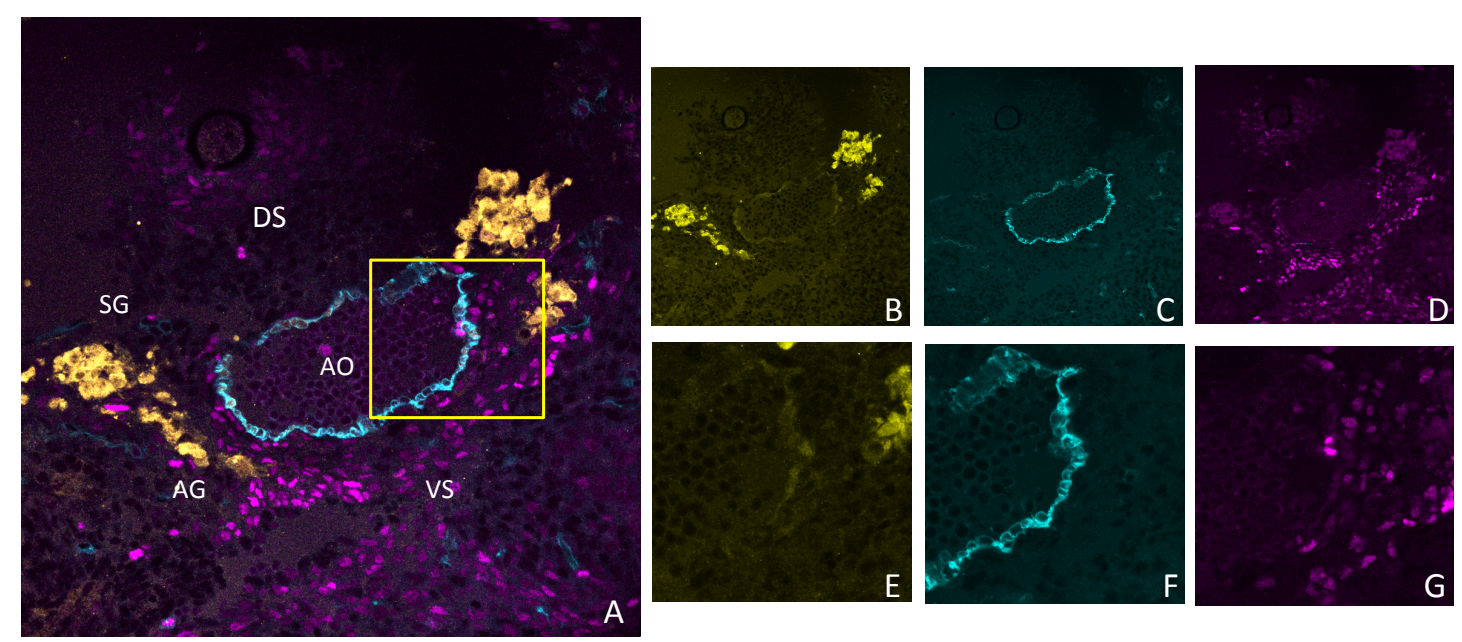

Figure 3-8: (A) section from a Gata3-GFP+ E11.5 embryo stained with (B) Gata3GFP (yellow), (C) CD34 (Cyan blue), (D) and Runx1 (purple). The yellow box highlights one of the areas where there is co-staining between all three markers. (E-G) Magnification of cells indicated by yellow box in image (A). Ao: dorsal aorta, AG: adrenal anlage, SG: sympathetic ganglia, DS: dorsal side, VS: ventral side. 


\section{3-3-2 E10.5:}

To further investigate HECs, the expression of CD34 and G3-GFP were analysed in E10.5 AGMs, at which point these cells, in contrast to E11.5, are more numerous (Swiers: 2013). The same populations of G3-GFP+ cells were identified (Figure 3-9 A,B). However, when we looked at the literature to see how the HE cells were isolated, Ve-Cad was an established marker for haemogenic endothelium, so at this point we switched to Ve-Cad as a marker for our endothelial cell population, especially since it gave a more robust signal in flow cytometry (Figure 3-9 C). In contrast to E11.5, around 3\% of the total G3GFP+ cells were mesenchymal cells, $1.4 \%$ were SNS cells, 2\% were haematopoietic cells (CD41/45), and 3-4\% were endothelial cells, the rest of the GFP+ cells are more likely to be a part of the developing nephric duct.

Interestingly, looking at IHC, G3-GFP expression was higher in the endothelium at this stage (Figure 3-9 B, C). What was even more intriguing was the presence of a transient haematopoietic population (CD41/45+) that was G3GFP+ and was present at E10.5 but disappeared at E11.5 (Figure 3-9 C). The majority of these cells did not express VE-Cad.

To test whether Gata3 expression in the endothelium marks haemogenic endothelial cells, we employed the OP9 co-culture system. 
A

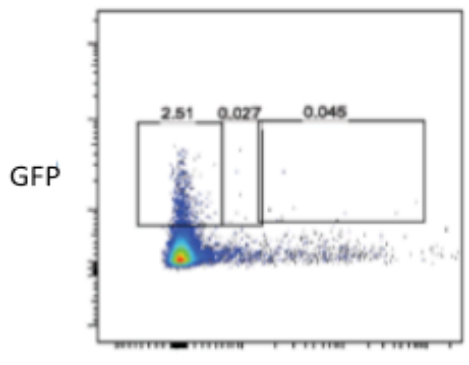

CD34

B
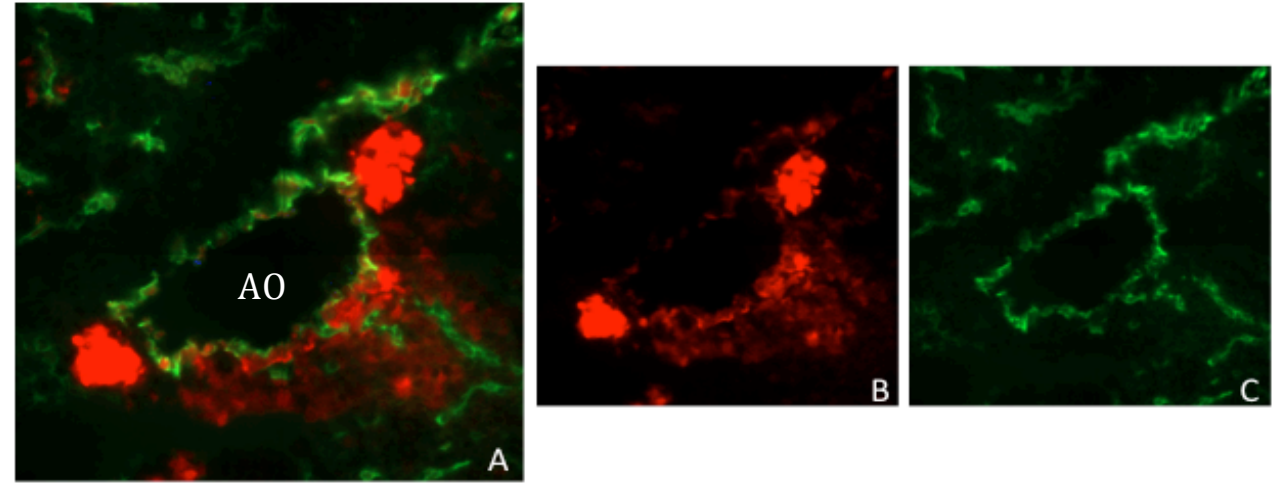

$$
\text { A }
$$
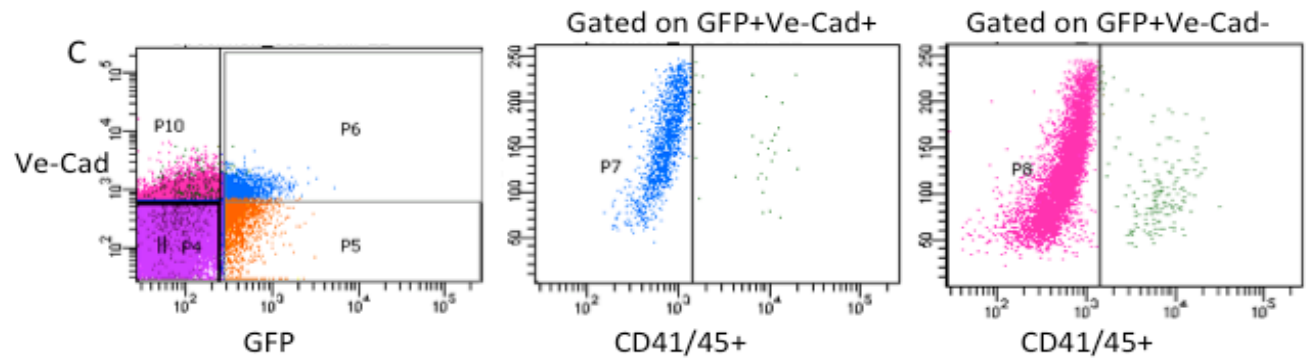

D
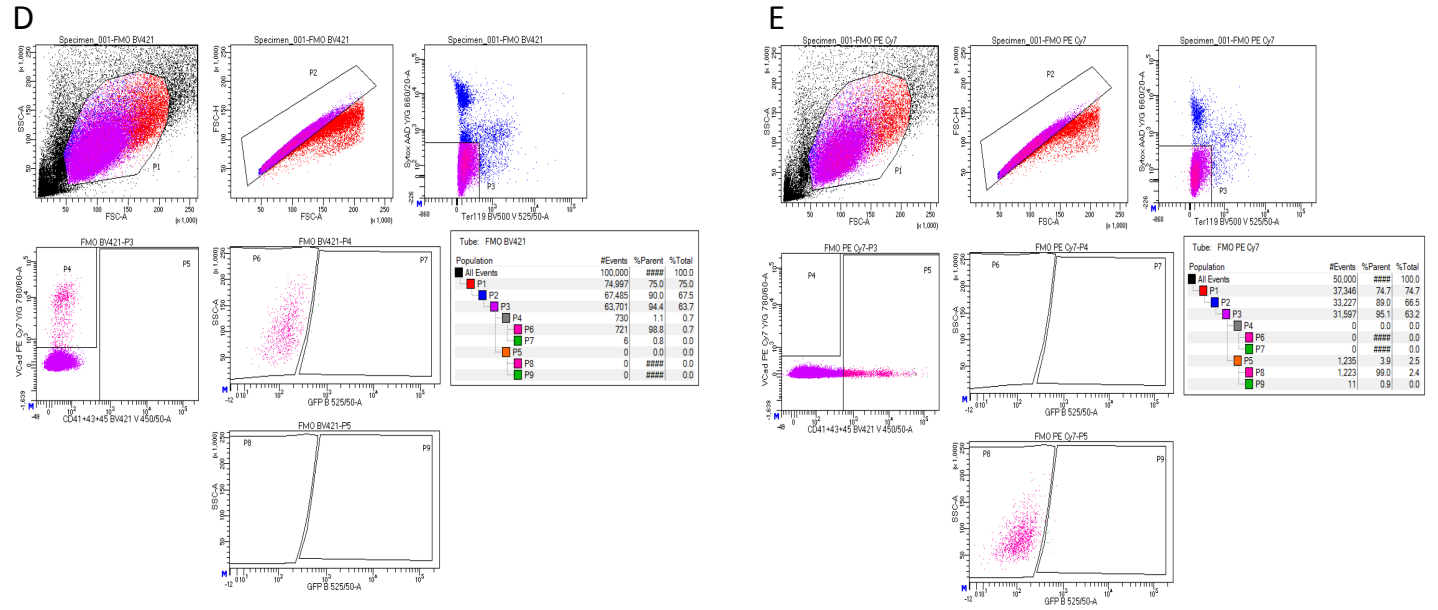

Figure 3-9: (A) Flow cytometry analysis of G3-GFP and CD34 on cells from E10.5 AGMs. GATA3 was expressed in endothelial cells. (B) section from a G3-GFP+E10.5 embryo stained for GFP (red) and CD34 (green). (C) Flow cytometry analysis of G3GFP, Ve-Cad and CD41/45 on cells from E10.5 AGMs, (D) FMO for CD41/43/45 on BV421 and (E) FMO for Ve-Cad on PE-CY7. FMO: Fluorescence minus one. 
3-4- The haemogenic endothelium activity is enriched in the G3+ compartment:

OP9 is a stromal cell line that was obtained from an osteoporotic OP/OP mouse calavaria (Kodama:1994).

These cells are used widely in developmental biology as a tool as they provide an easy, versatile and efficient culture system to support the growth, differentiation and proliferation of haematopoietic cells from different sources of stem cells based on the growth factors supplied.

Cells were obtained from E9.5-E10.5 G3-GFP embryos, since the haemogenic endothelium activity is higher at that stage compared with E11.5. Cells were then cultured on top of OP9 in media supplemented with IL7, SCF and Flt3. Cells were harvested after 3 weeks and analysed using either flow cytometry analysis or CFU-C assays (Figure 3-10).

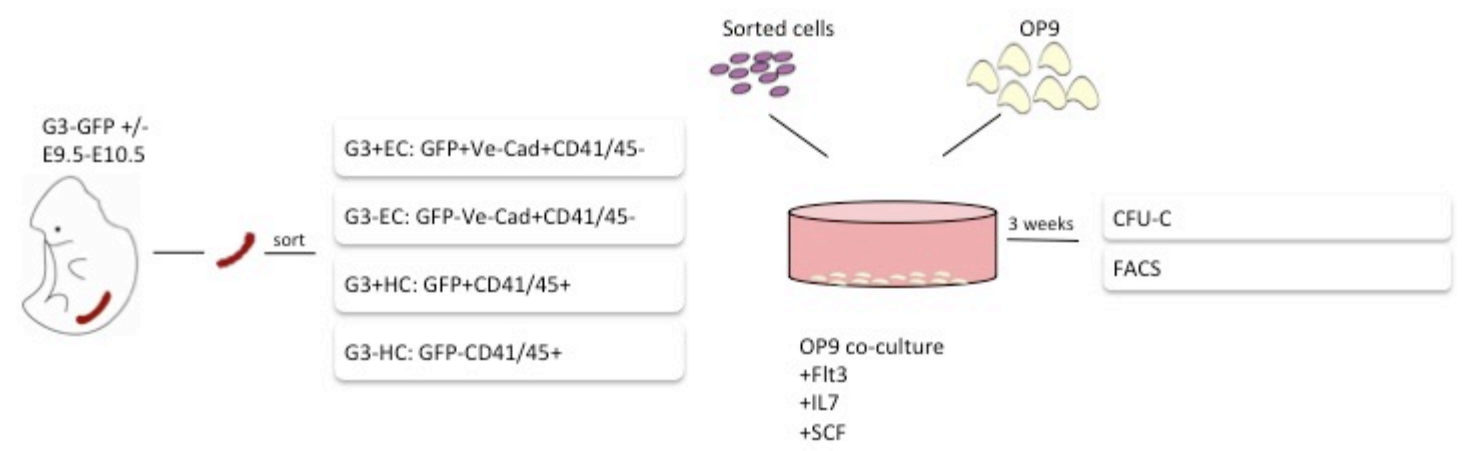

Figure 3-10: Schematic diagram for experimental approach for OP9 co-culture. AGMs are dissected from E9.5-E10.5 G3-GFP+ embryos, and made into single cell suspension. Cells are then sorted into four populations, and plated on top of OP9 in conditions that support the production of haematopoietic progeny. Cells are collected after three weeks to be analysed by flow cytometry and plated in methylcellulose for CFU-C assays.

Colony Forming Unit-Cells (CFU-C) is an in-vitro culture assay, aimed at identifying the haematopoietic potential of a tissue; i.e. the ability of haematopoietic progenitors to proliferate and differentiate, as detected through the formation of haematopoietic colonies in methylcellulose medium in the 
presence of cytokines. The number of colonies relates to the frequency of these cells within a haematopoietic tissue.

Haematopoietic progenitors can give rise to different types of identifiable colonies in CFU-C assays. CFU-Mix colonies are derived from the most immature and stem cell-like multipotent progenitor and contain granulocytes, macrophages and erythrocytes. Next, there are CFU-GM colonies, which are derived from more mature progenitors than CFU-Mix and contain granulocytes and macrophages only. CFU-G colonies are derived from the precursors for granulocytes, CFU-M from the precursors for macrophages and BFU-E are the burst-forming unit for erythrocytes.

Initially, we sorted the AGM cells into 4 populations: G3+EC: GFP+VeCad+CD41/45-, G3-EC: GFP-Ve-Cad+CD41/45-, and used Mesenchymal and SNS cells (GFP+Ve-Cad-CD41/45-) for negative control and CD45+ cells for positive control. We plated 1000 cells per well and the following CFU-C readout was obtained (Figure 3-11). The G3+EC output was five fold higher than that of G3EC. As expected, the highest colony count came from the haematopoietic cells, which served as our positive control here, and no colonies grew from the MC/SNS compartment, which served as a negative control in this experiment.

Then, given that 1000 cells from G3+EC population were about 8-12 embryo equivalent (ee), depending on the embryonic age and the variation of G3+EC count in each embryonic stage E9.5-E10.5, whereas 1000 cells from G3EC were less than 0.5 ee, we changed our strategy to make it more biologically representative. However, plating less than a 1000 cells of G3+EC did not give any read-out.

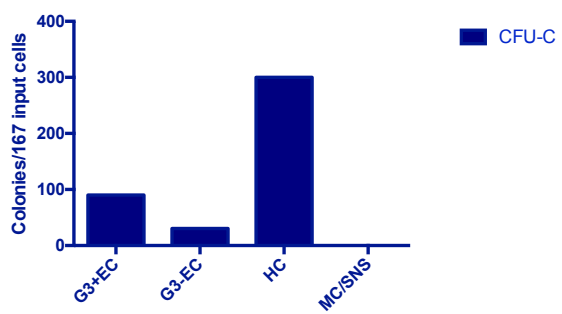

Figure 3-11: CFU-C assay representing the progenitor counts of G3+EC, G3-EC, HC and MC/SNS cells plated into methylcellulose after OP9 co-culture. $n=1$. 
So we choose to plate 1000 cells of G3+EC and their ee of G3-EC. Here we introduced a new sorting strategy to also include G3+HC in order to test their haematopoietic potential. The new sorting strategy was: G3+EC: GFP+VeCad+CD41/45-, G3-EC: GFP-Ve-Cad+CD41/45-, G3+HC: GFP+CD41/45+, and G3HC: GFP-CD41/45+ (Figure 3-12). At the end of the co-culture, we analysed the harvested cells using flow cytometry and CFU-C assays (Figure 3-13 and 3-14).

The haematopoietic potential in the G3+ endothelial compartment was significantly higher than that of the G3-EC as was revealed by both CFU-C and flow cytometry analysis.
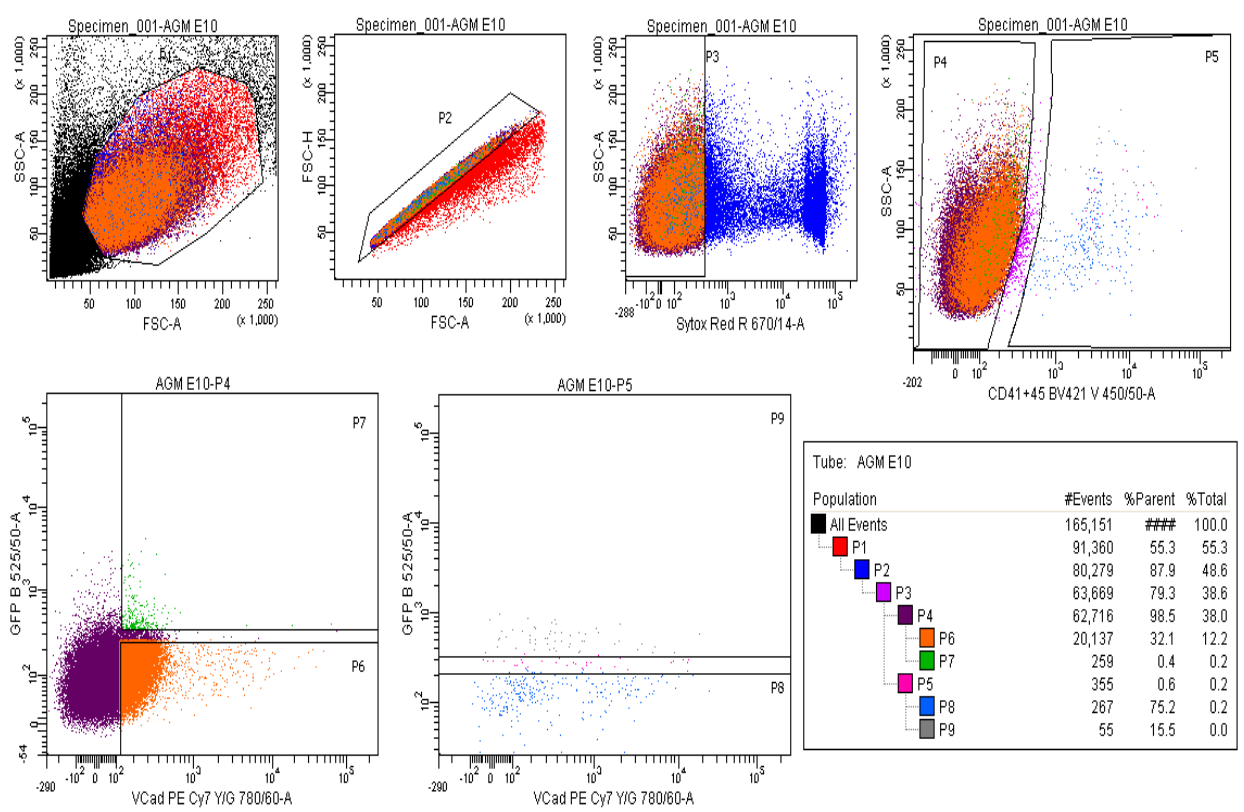

Figure 3-12: Sorting Strategy for the OP9 co-culture: combining GFP, Ve-Cad (on PE Cy7), CD41/45 (BV421) has enabled us to sort four distinct populations from the AGM. Gating strategy of representative AGM sample on a BD ARIA sorter operating on BD FACSDIVA Software. P1: Morphology gate, P2: single cells, P3: Live cells, P4: CD41/45- cells, P5: CD41/45+ cells, P6: G3-EC: CD41/45-GFP-Ve-Cad+, P7: G3+EC: CD41/45-GFP+Ve-Cad+, P8: G3-HC: CD41/45+GFP-, and P9: G3+HC: CD41/45+GFP+

In the flow cytometry analysis, both G3+EC and G3-EC were shown to be able to produce haematopoietic progeny (CD45+ cells) that expressed mature myeloid markers (CD11b and Gr1), although the haematopoietic output from G3+EC was more robust (Figure 3-13). 
The CFU-C results were consistent with those of the flow cytometry analysis, and G3+EC produced significantly higher numbers of colonies in contrast to G3-EC (Figure 3-14).

It should be noted here, while the plating of the OP9 co-culture was based on ee count, the amount of cells of the G3-EC were consistently higher by a factor of ten than G3+ECs.

A
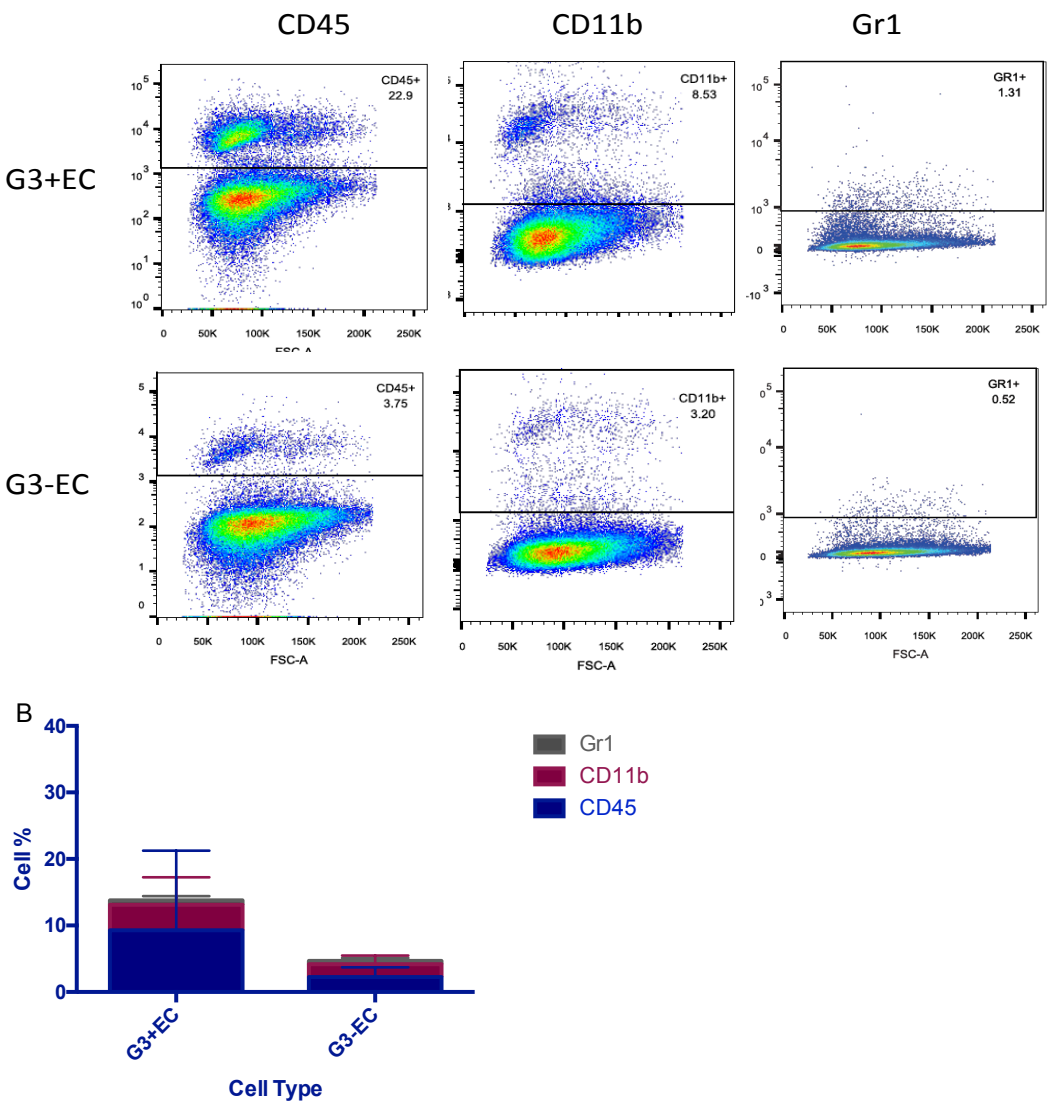

Figure 3-13: (A) FACS analysis of the haematopoietic progeny produced by both G3+EC and G3-EC after OP9 co-culture. CD45 marks all the haematopoietic cells, $C D 11 b$ and Gr1 mark cells of the myeloid lineage. (B) Total percentage of CD45, CD11b and Gr1 cells in FACS analysis represented by bar graph. Statistical analysis were done using two-way ANOVA, $n=4$. 


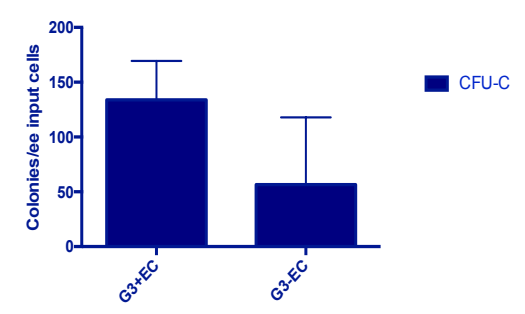

Figure 3-14: CFU-C assay representing the progenitor counts of G3+EC and G3-EC after OP9 co-culture. Statistical analysis were done using paired t test, $n=4$.

3-5- G3+ progenitors are enriched for CFU-Mix potential, the most immature and stem cell-like progenitor:

To further investigate the haematopoietic potential of the previously identified G3+ haematopoietic cells (3-3), we collected those cells, along with their G3-HC counterparts and cultured them on OP9 cells as above.

Flow cytometry analysis revealed that both G3+HC and G3-HC can produce haematopoietic progeny (Figure 3-15 A,B). It also should be noted here that the $\mathrm{G} 3+\mathrm{HC}$ population comprised $0.5-1.5 \%$ of the present total haematopoietic population in the AGM, whereas G3-HC make up the rest of that population.

Intriguingly, while G3-HC produced over $5 \mathrm{x}$ the colonies that the $\mathrm{G} 3+\mathrm{HC}$ produced, the colonies that were generated were mostly CFU-M, CFU-GM and some CFU-Mix (Figure 3-15 C-F). However, around 90\% of the colonies produced by G3+HC were CFU-Mix colonies, generated by the most immature and stem cell-like progenitor (Figure 3-15 C-G). 
A
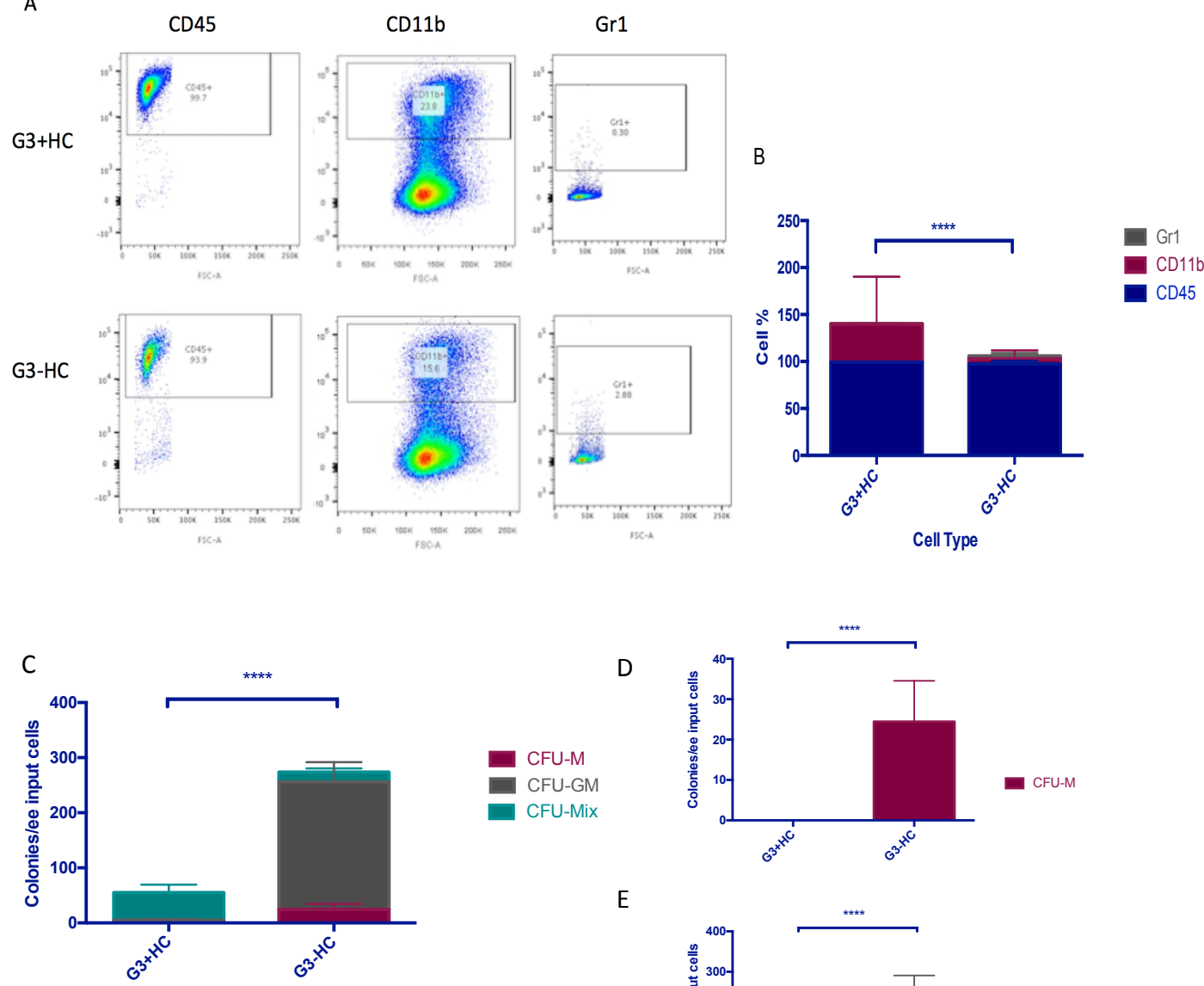

G

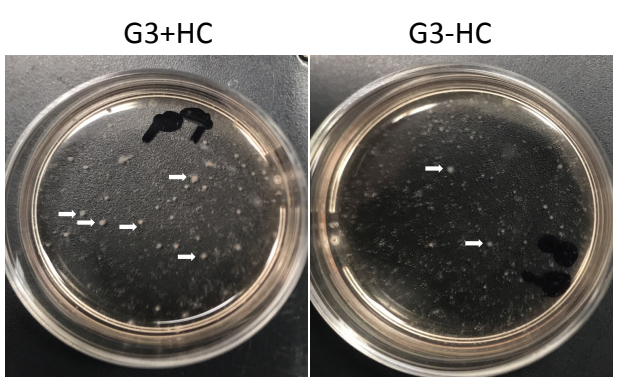

$E$

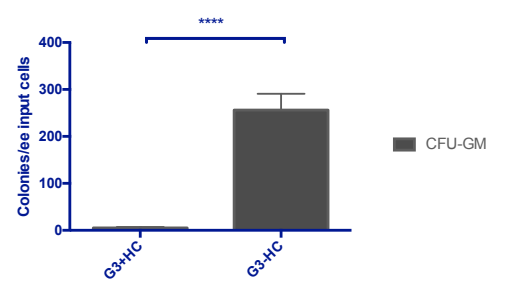

$\mathrm{F}$

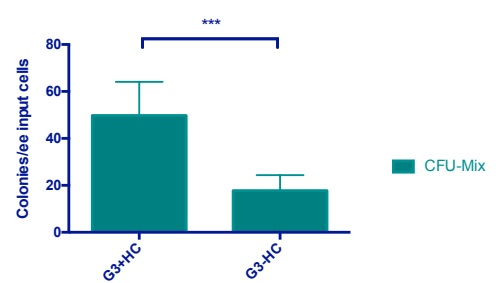

Figure 3-15: G3+ progenitors are enriched for CFU-Mix potential, the most immature and stem cell like progenitor: $\mathrm{G} 3+\mathrm{HC}$ and $\mathrm{G3}-\mathrm{HC}$ were sorted from E9.510.5 G3-GFP embryos and cultured on OP9 cells as ee. (A) Flow cytometry analysis of the haematopoietic progeny produced by both G3+HC and G3-HC. CD45 marks all the haematopoietic cells, CD11b and Gr1 mark mature myeloid cells.(B) Total percentage of CD45, CD11b and Gr1 cells in FACS analysis represented by bar graph (D-F): $C F U-C$ assay representing the progenitor counts of G3+HC and G3-HC after OP9 co-culture; (B) represents the total count of progenitors. Separate panels show bar graphs representing different types of colonies produced in the CFU-C assay where (D) CFU-M, (E) CFU-GM, and (F) CFU-Mix. (G): picture of CFU-C plates, showing a higher number of CFU-Mix colonies, marked by arrows, produced by the $\mathrm{G} 3+\mathrm{HC}$ in contrast to G3-HC. Statistical analysis were done using two-way ANOVA for $B$ and $C$, and paired $t$-test for $D-F . n=3, * * *: P=0.0003, * * * *: P<0.0001$ 
3-6- Haematopoietic progenitors are restricted to the G3-HC compartment:

We then wanted to determine the origin of both $\mathrm{G} 3+\mathrm{HC}$ and G3-HC, so we performed CFU-Cs with directly sorted cells from G3-GFP E10.5 AGMs (Figure 312). Interestingly, the only population that produced haematopoietic progenitors was the G3-HC, while G3+HC did not produce any, indicating either the absence of haematopoietic progenitors in this compartment, or that the frequency of haematopoietic progenitors in the sorted cells is too low to give a read-out at this point. As expected, neither endothelial population (G3+EC and G3-EC) produced any colonies when plated directly in methylcellulose (Figure 3-16).

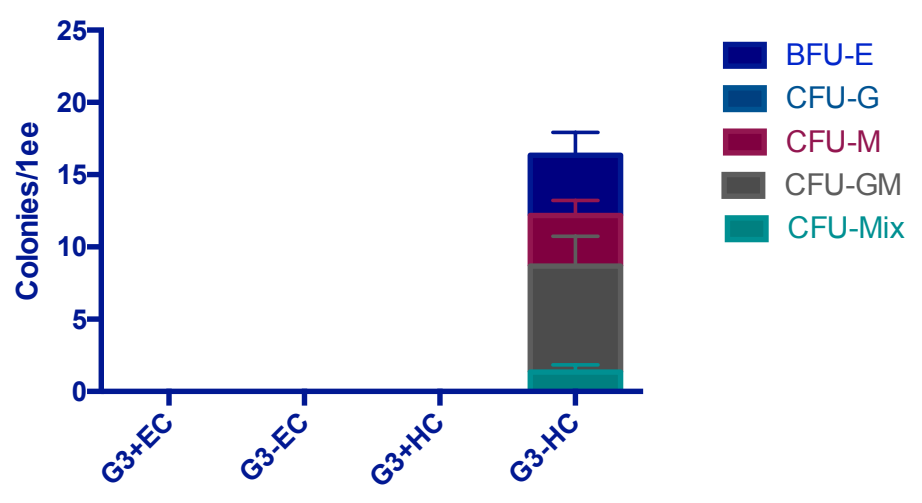

Figure 3-16: CFU-C assay representing the progenitor counts of freshly sorted $G 3+E C, G 3-E C, G 3+H C$ and G3-HC that were directly plated uncultured into methylcellulose. The only population that was able to generate colonies directly was that of G3-HC. $n=1$.

\section{3-7- G3 marks specific stages along the developmental pathway} towards the generation of dHSCs:

As our results suggested that Gata3 is expressed in haemogenic cells, we wanted to determine more precisely the type of HSC precursor that is marked by Gata3. The OP9 co-aggregates is a system developed by Rybtsov et al (2011) to support the maturation of pre-HSCs into definitive HSCs in culture. The system co-aggregates sorted cells from the AGMs with OP9 stromal cells in conditions 
that favour the maturation of pre-HSCs into definitive HSCs. In contrast to AGM explants, this system allows more cell manipulation and further support. Moreover, additional studies by the authors have demonstrated the superiority of OP9 cell co-aggregates to AGM re-aggregates in terms of supporting HSCs production. Most importantly, based on the developmental stage and specific cell surface markers, distinct steps in HSC maturation can be distinguished (Rybtsov: $2011 ; 2014)$.

AGMs were obtained from E9.5-10.5 and E11.5 G3-GFP embryos, made into single cell suspensions and sorted into four populations (G3+EC, G3-EC, G3+HC and G3-HC; Figure 3-17, 3-18). We changed the sorting strategy here and added two more markers: Ter119 a marker for erythrocytes (exclusion marker) and CD43 which is another marker for Pre-HSCs (Figure 3-18). Cells are then coaggregated with OP9 stromal cells in media supplemented with Flt3, IL6 and SCF, which are conditions that are known to promote pre-HSC maturation. Coaggregates are then harvested and either transplanted into irradiated recipients, plated into methylcellulose for CFU-C assays, or analysed using flow cytometry (Figure 3-17).

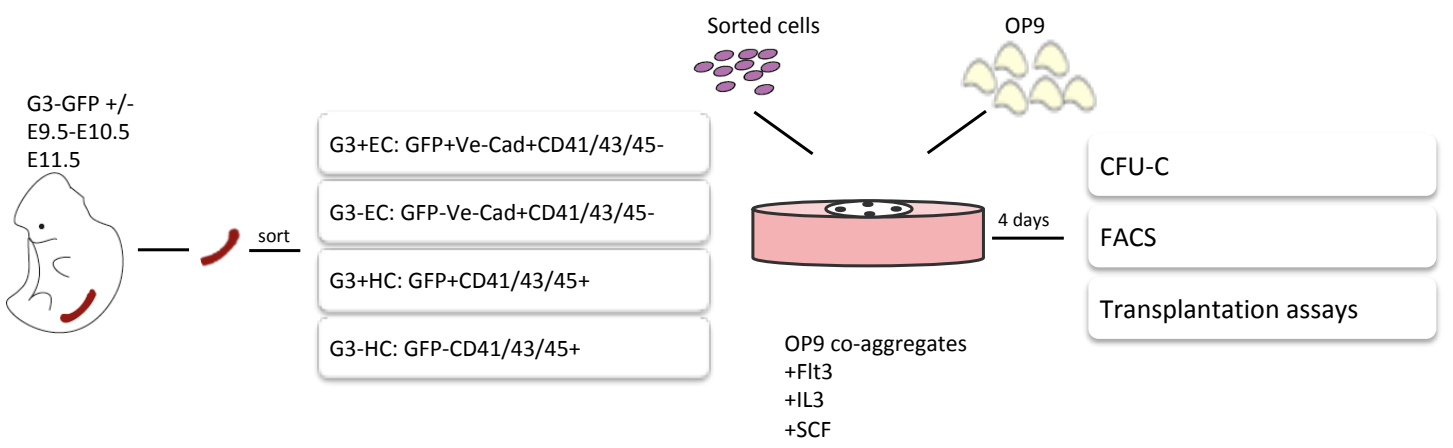

Figure 3-17: Schematic diagram for experimental approach for OP9 co-aggregates. AGMs are dissected from E9.5-E10.5 and E11.5 G3-GFP+ embryos, and made into single cell suspension. Cells are then sorted into four populations, co-aggregated with OP9 cells and the aggregates cultured in conditions that favour HSC production. Cells are collected after 4 days to be transplanted, analysed by flow cytometry, or plated in methylcellulose for $C F U-C$.

We looked for the presence of haematopoietic cells by flow cytometry analysis after co-aggregations. The results were mostly consistent with those of 
the CFU-C assays, referring to section 3-6, however, it revealed that the G3+ compartments tended to generate a higher percentage of haematopoietic cells (Figure 3-19 A).
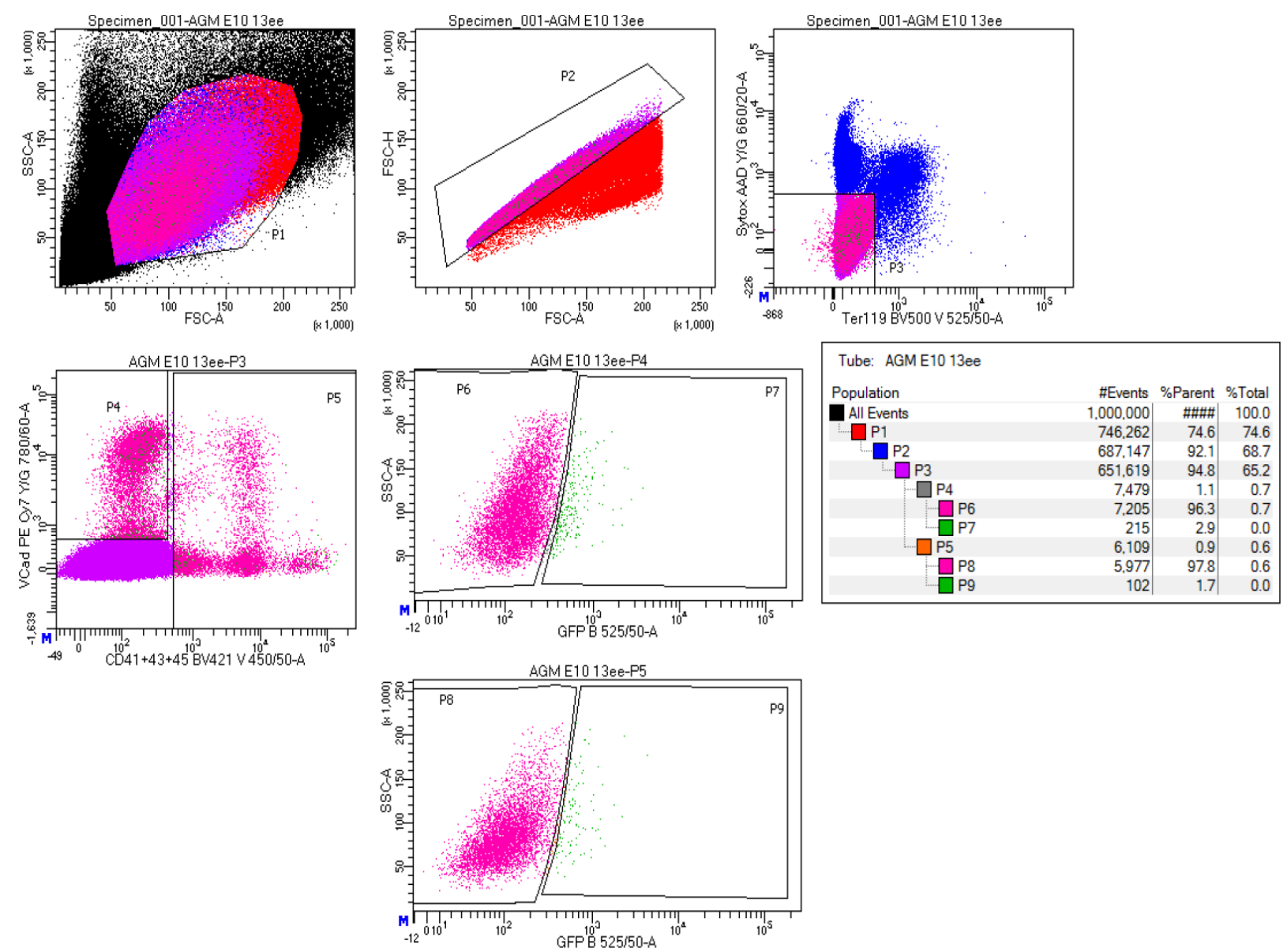

Figure 3-18: Sorting Strategy for the OP9 co-aggregates: combining Ter119, GFP, Ve-Cad, CD41/43/45 has enabled us to sort four distinct populations from the AGM. Gating strategy of representative AGM sample on a BD ARIA sorter operating on BD FACSDIVA Software where; P1: morphology, P2: single cells, P3: Ter119- live cells, P4: Ter119-Ve-Cad+CD41/43/45-, P5: Ter119-CD41/43/45+, P6: G3-EC: Ter119-Ve-Cad+GFP-CD41/43/45-, P7: G3+EC: Ter119-Ve-Cad+GFP+CD41/43/45-, P8: G3-HC: Ter119-Ve-cad+/-GFP-CD41/43/45+, and P9 :G3+HC: Ter119-Ve$\mathrm{Cad}+/-G F P+C D 41 / 43 / 45+$.

In CFU-C assays after the co-aggregation, the G3-HC progenitor counts have increased by a two fold change in contrast to G3-HC after the plating of a fresh sorted cells (3-16). Interestingly, the G3+HC have actually expanded to comparable levels to that of G3-HC. Both G3+EC and G3-EC produced a small number of colonies with the G3-EC being the lower of the two (Figure 3-19 B). 
In transplantation assays, only two mice highly repopulated in the G3+HC compartment for cells obtained from E9-5-E10.5 AGMs at the one-month analysis. However, before the four-month bleed and flow cytometry analysis, one of those two mice died. The arrow denotes the flow cytometry result obtained after 1 month following the transplantation. However, given its high levels, it is highly likely that this mouse would have remained highly repopulated in the ensuing three months (Figure 3-20 A).
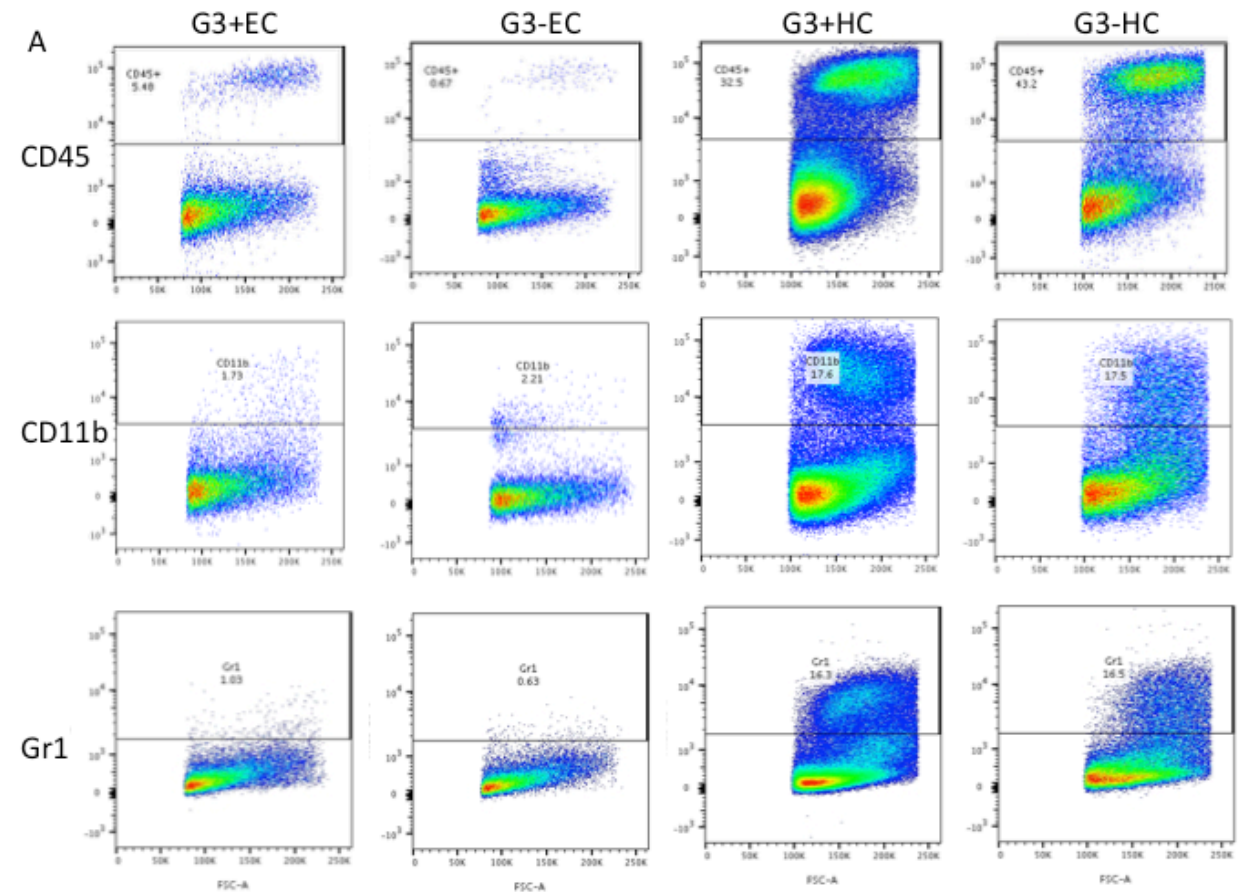

B

C
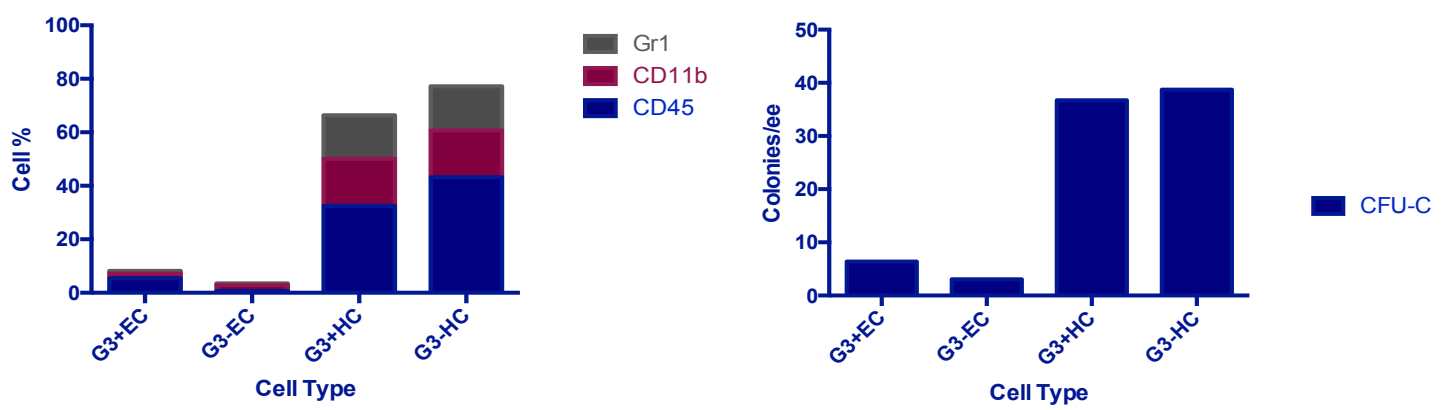

Figure 3-19: (A) Flow cytometry analysis of the haematopoietic progeny produced by G3+EC, G3-EC G3+HC and G3-HC obtained from E9.5-E10.5 G3-GFP embryos after OP9 co-aggregates. CD45 marks all the haematopoietic cells, CD11b and Gr1 mark cells of the myeloid lineage. (B) Total percentage of CD45, CD11b and Gr1 cells in FACS analysis represented by bar graph (C) CFU-C assay representing the progenitor counts of G3+EC, G3-EC, G3+HC and G3-HC plated into methylcellulose after co-aggregate culture. $n=1$ 
We also performed transplantation assays after co-aggregates from cells obtained from E11.5 AGMs. Interestingly, at this stage, the only population that had repopulated the irradiated recipients was that of G3-HC, where three mice repopulated (Figure 3-20 B).
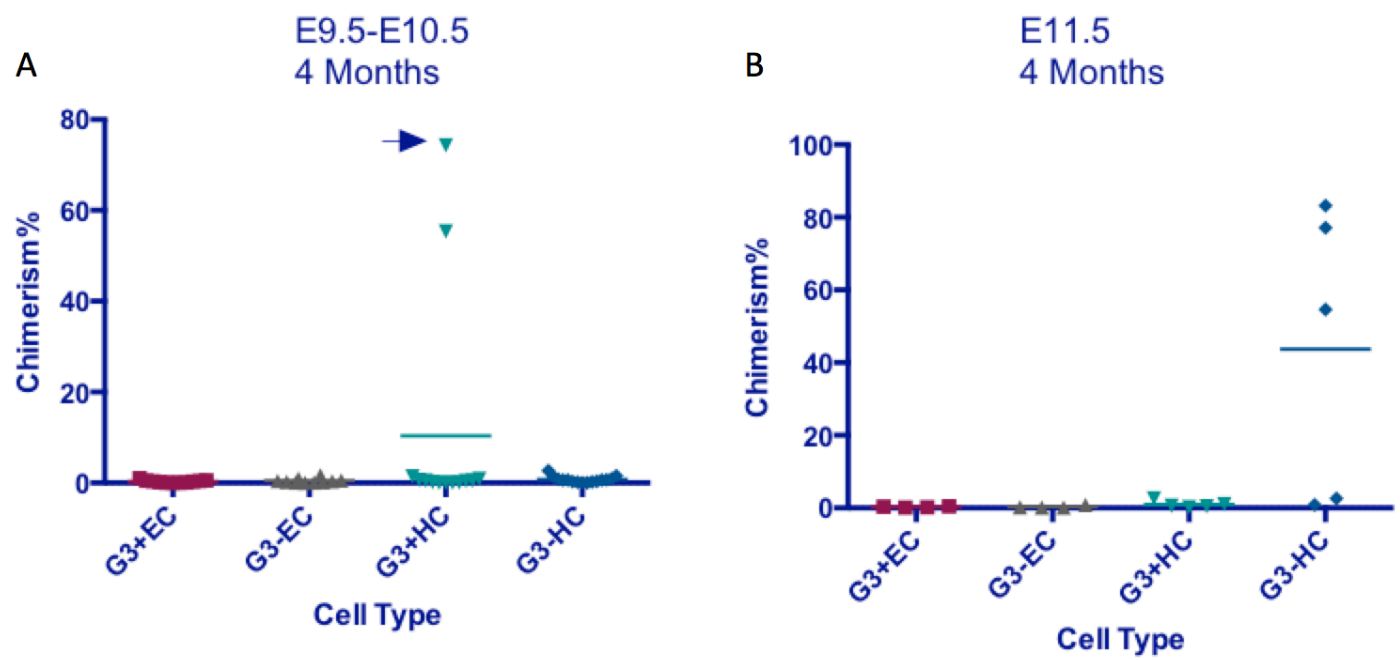

Figure 3-20: (A) Chimerism levels in transplanted animals from sorted cells (G3+EC, G3-EC, G3+HC and G3-HC) obtained from E9.5-E10.5 G3-GFP AGMs and coaggregated with OP9-cells. Horizontal solid line represents the mean, and the arrow represents the 1 month result for one mouse. (B) Chimerism levels in transplanted animals from sorted cells (G3+EC, G3-EC, G3+HC and G3-HC) obtained from E11.5 G3-GFP AGMs and co-aggregated with OP9-cells in ee. Horizontal solid line represents the mean. Chimerism of $5 \%$ or higher was set as the threshold at which mice are considered positive for repopulation. E9.5-10.5 $n=28, E 11.5 n=18$.

\section{3-8-Discussion:}

Flow cytometry analysis showed that G3-GFP is expressed in CD34+ endothelial cells, p75+ SNS cells and PDGFRb+ mesenchymal cells at E11.5 within the AGM region of the embryo. However, it was not expressed in CD45+ haematopoietic cells at that stage. This indicates that Gata3 is not expressed in haematopoietic cells of the AGM region, including HSCs. This corresponds with what was suggested before by Fitch et al. (2012) and implicates Gata3 as a factor influencing the haematopoietic environment. 
Interestingly, Gata3 was expressed within a subgroup of hematopoietic cells at E10.5. This population proved to be a transient population, starting to appear at late E9, with its highest levels at E10.5 and declining the older the embryo gets until it finally disappears at E11.5. This corresponds with what was reported before by Swiers et al. (2013), and suggests a role for Gata3 in haematopoietic progenitor development.

The flow cytometry analysis and IHC staining has also revealed that Gata3 is expressed in a subpopulation of mesenchymal cells (Figure 3-2, 3-4 B,C,E,). Other key regulators of HSCs are also expressed in the mesenchyme such as Runx1 (North: 1999) and Bmp4 (Durand: 2007). However, the identity and function of these cells is still unknown. The G3-GFP mouse line now presents me with a tool to isolate and further characterise these mesenchymal cells.

Gata3 is expressed in a subgroup of endothelial cells, where it co-stains with Runx1. It has been reported that Gata3 expression is elevated in HE, along with other transcription factors that have been associated with early specification of the haematopoietic system such as Gata2, Meis1 and Runx1. This upregulation was obvious from E8.5 (Swiers: 2013). Gata3 expression was found in endothelial cells in flow cytometry analysis at E10.5 which is followed by a rapid reduction the older the embryos get (Figure 3-2, 3-4 A,C). This timeline also corresponds with the function of haemogenic endothelium and HSC and progenitor generation.

To examine the spatial distribution of the G3-GFP+CD34+ population in the walls of the dorsal aorta, IHC on G3-GFP-expressing embryo sections was performed. Few Gata3-GFP+ cells were found within the endothelial layer that forms the walls of the dorsal aorta within the AGM region at E11.5. This was consistent with the AGM being considered one of the haemogenic endothelium sites, where Gata3 is upregulated in the AGM at the time of HSC emergence (Mascarenhas: 2009) and Gata3 was reported to be enriched in HE (Swiers: 2013). Its upregulation seems to start already at E8.5 at the time of haematopoietic specification in HE (Swiers: 2013). Together, these data suggest 
that Gata3 plays an additional role in HSC development independent of its expression in the SNS (Fitch: 2012).

G3-GFP is also expressed in SNS cells, identified here by flow cytometry as G3-GFP+P75+ cells, in the AGM. Immunohistochemistry on G3-GFP E11.5 embryo sections showed that Gata3 expression overlapped with Th staining in the SNS cells surrounding the AGM region (Figure 3-4 A,H,I,J), and that staining overlapped with P75, indicating that P75 can act as a membrane marker to FACS sort SNS cells more directly (Figure 3-7 A-G).

Gata3 is a member of a transcription factor network that controls sympathetic neuron development from their precursors in the neural crest. In fact, Gata3 is essential for the generation and differentiation of sympathetic neurons and the production of catecholamines (Tsarovina: 2010). It was previously reported that Gata3's role in HSC development is secondary to its role in the SNS; i.e. Gata3 influences HSC through the SNS and catecholamine release (Fitch: 2012). However, the findings presented here suggest that Gata3 may also impact on HSC development through its expression in HE, MCs and HCs.

In OP9 co-cultures, supplemented with factors that support HSPC generation, G3+EC and G3-EC obtained from the AGM of E9.5-E10.5 G3-GFP embryos gave rise to haematopoietic progeny, including mature myeloid cells. This confirms the presence of HE cells within the AGM region of embryos which was reported previously (Boisset: 2010, Bertrand 2010, Kissa 2010, Swiers: 2013). However, a single cell OP9 co-culture would still be needed to confirm that.

Here we have shown that G3+EC are haemogenic, and that G3+EC haemogenic potential is more robust than that of G3-EC, which corresponds with our previous findings from IHC of Gata3 being co-expressed with both Runx1 and CD34 in the walls of the dorsal aorta.

In addition, here we have shown that G3+HC not only are capable of generating robust haematopoietic progeny in OP9 co-cultures, but also that they mainly generate CFU-C Mix in CFU-C assays, the most immature stem-cell like 
colony. Therefore, we wanted investigate these cells in more detail and identify their place within the HSC developmental hierarchy.

We performed CFU-Cs directly for our sorted populations, which revealed that the G3+HC could not produce progenitors when plated directly into methylcellulose, unlike G3-HC. We then performed OP9 co-aggregates which is a method that was optimised to facilitate the maturation of pre-HSCs into dHSCs. After the co-aggregates, G3+HC produced colonies that were one fold higher than those produced by G3-HC prior to the co-aggregates (their levels were similar after the co-aggregates), and was able to produce more haematopoietic cells (as shown by flow cytometry) than those produced by G3-HC. Furthermore, the only population of AGMs obtained from E9.5-E10.5 G3-GFP embryos that repopulated irradiated recipient mice was that of G3+HC.

All of that suggests that the G3+HC could contain a pre-HSC population, corresponding to the ones described by Rybtsov et al $(2011,2014)$. However, as all haematopoietic markers (CD41/43/45) were combined in a single colour, we do not currently know if it is type I or II, or if it is even pro-HSCs.

We also wanted to confirm that HSCs are not in the G3+, as previously suggested (Fitch: 2012). We therefore sorted AGMs from E11.5 G3-GFP embryos into two populations: GFP+ and GFP- in order to minimise the cell loss. We tried injecting mice with 1ee, 2 ee, 3 ee and 4 ee. However, there was no repopulation from either compartment, suggesting technical problems in the assay, possibly due to low HSC numbers in the AGMs as they were haploinsufficient (due to the GFP knockin). Reduced HSC numbers in Gata3 heterozygous AGMs were previously demonstrated (Fitch:2012).

To circumvent this problem, we used the co-aggregate system for our four populations obtained from E11.5 G3-GFP AGMs. Interestingly, unlike E10.5, the only group that showed repopulation was that of G3-HC. This suggests the presence of dHSCs in that compartment, which corresponds with what was reported before by Fitch et al (2012) that Gata3 is not expressed in dHSCs in the AGM. 
The fact that there were only low numbers of haematopoietic progeny produced by either G3+EC or G3-EC in the co-aggregate system, even though this is similar to co-cultures to some extent in terms of growth factors and OP9 cells, could be contributed to the duration of the co-aggregates (4 days) and that there is not sufficient time for the EHT to take place, compared to that of OP9 cocultures (3 weeks).

In the next chapter, we will focus more on the HE and Gata3 and whether Gata3 actually performs a functional role in the HE. We will investigate the conditional deletion of Gata3 from the endothelium and the effect it has on HSCs and progenitors. 


\section{4- GATA3 plays an important role in the haemogenic endothelium}

\section{4-1- Background:}

Gata3 was previously reported to be expressed in endothelial cells, mesenchymal cells (Manaia: 2000) and sympathoadrenal cells within the AGM region (Fitch: 2012) and has subsequently been shown to be enriched in haemogenic endothelial cells and is expressed in haematopoietic progenitor cells (Swiers 2013). This was also confirmed using our Gata3-GFP reporter mouse line in chapter 3.

It was established that Gata3 regulates HSC and progenitor production by means of the SNS (Fitch: 2012). However, its function in the other cell populations is still to be investigated.

Previously, it was reported by the Ottersbach group that Gata3 deletion causes a significant reduction in HSCs and CFU-S progenitor cells in E11.5 and E10.5 AGMs. When AGMs from E11-E11.5 embryos of wild-type embryos $(\mathrm{G} 3+/+)$, Gata3 heterozygous embryos (G3+/-), and total knockout embryos (G3/-), were transplanted into irradiated recipients, there was a noticeable reduction in the repopulating ability of both G3+/- and G3-/- AGMs. Around 60\% of G3+/+ embryos showed a donor contribution, compared to 17\% in G3+/- and 14\% in G3-/-. Interestingly, when adding an explant step prior to the transplant, the repopulating ability of G3+/- AGMs was restored to that of a G3+/+; however, the observed reduction persisted in G3-/- AGMs. The effect of Gata3 deletion on more mature haematopoietic progenitor cells is yet to be investigated.

The findings described above had all been made using germline deleted Gata3 embryos, and the haematopoietic phenotype had been largely attributed to the failure of the defective SNS to secrete catecholamines (Fitch: 2012). However, given that HSCs and progenitors emerge from vascular endothelial cells through the formation of intra-aortic clusters (Jaffredo: 1998), and that Gata3 is expressed within those cells at the time of haemogenic activity of those 
cells, it is possible that the haematopoietic defect is caused by the absence of Gata3 in more than one cell type. We, therefore, wanted to look at the effect of the conditional deletion of Gata3 from the vascular endothelium on HSC and progenitor formation, using Vec-Cre-mediated deletion (Chen: 2009).

Vec is a transmembrane protein that is involved in endothelial cell adhesion (Lampugnani: 1995) and, from E9.5, it is expressed in the vascular endothelium throughout the embryo (Drake: 2000), and also marks haematopoietic cells at that stage (North: 2002; Taoudi: 2004). This expression pattern persists at later developmental stages, and Vec has been reported to mark haemogenic endothelial cells, pre-HSCs and definitive HSCs at E10.5 and E11.5 (Ivanovs: 2014; North: 2002; Taoudi: 2005; Rybtsov: 2011; Rybtsov: 2013).

Using a Vec-Cre transgenic mouse line (Chen: 2009) we were able to excise Gata3 from the endothelium and investigate the effect of that deletion on HSC and haematopoietic progenitor generation.

\section{4-2- Aims:}

Investigate the effect of Gata3 deletion on haematopoietic progenitors

- Identify the effect of the conditional deletion of Gata3 from endothelium on haematopoietic progenitors

- Identify the effect of the conditional deletion of Gata3 from endothelium on haematopoietic repopulation 


\section{4-3- Gata3 deletion causes a reduction in haematopoietic progenitor}

cell counts in E10.5 and E11.5 AGMs in a dose-dependent manner:

Our group has previously reported a requirement for Gata3 in HSC production in the AGM (Fitch: 2012); however, its role in haematopoietic progenitors in the AGM was unknown. As we had detected Gata3-GFP+ haematopoietic cells in the E10.5 AGM (Figure 3-5C), we wanted to investigate whether Gata3 deletion has an effect on HSPCs. AGMs were collected from Gata3 wild-type (G3+/+), Gata3 heterozygous (G3+/-), and total knockout embryos (G3-/-), dissociated and plated in methylcellulose. In some cases, AGMs were placed into explant culture prior to plating in methylcellulose medium, as this had previously been shown to rescue HSC numbers in G3+/- AGMs (Fitch: 2012) (Figure 4-1). The number and types of colonies were scored after 7 days for each genotype and compared to the control condition; i.e. the wild-type genotype. It should be noted here that some of the knockout embryos were smaller in size in contrast to their wild-type and heterozygous littermates. However, as they were harder to obtain, only one knockout embryo would survive in every 2-3 litters, those smaller embryos were used.

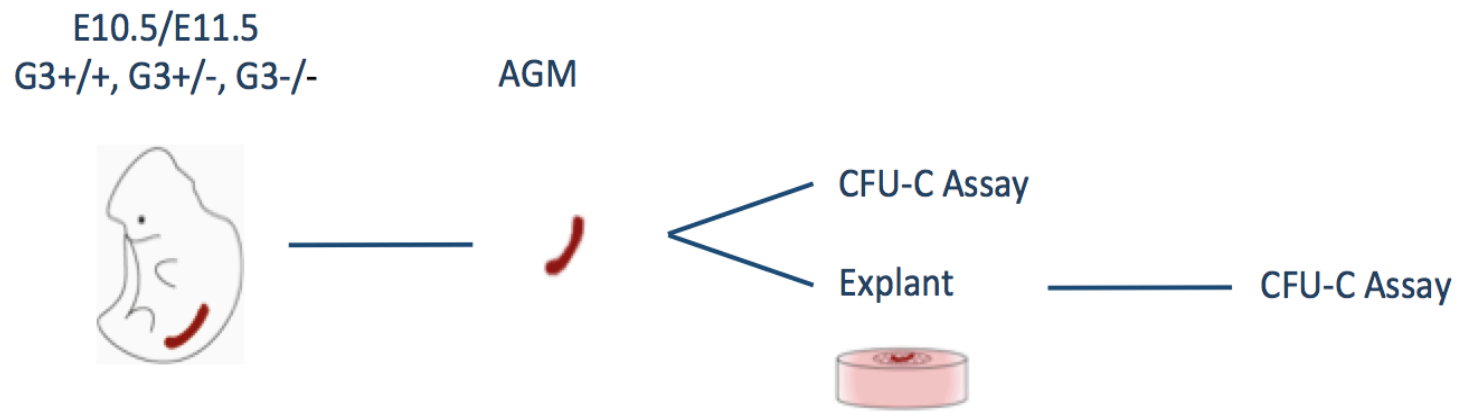

Figure 4-1: Representative schematic of the experimental design of the CFU-C assay. AGMs are dissected from E10.5 or E11.5 G3+/+, G3+/-, G3-/- embryos, and either dissociated and plated into methylcellulose medium directly, or cultured in explant cultures at the air/liquid interface, before plating in methylcellulose medium.

In contrast to wild-type embryos $(\mathrm{G} 3+/+)$, the haematopoietic progenitor count was lower by $22 \%$ in heterozygous embryos (G3+/-) in E11.5 AGMs. This 
reduction was more pronounced in the AGMs of knockout embryos (G3-/-) by a significant 35.7\% (Figure 4-2:A).
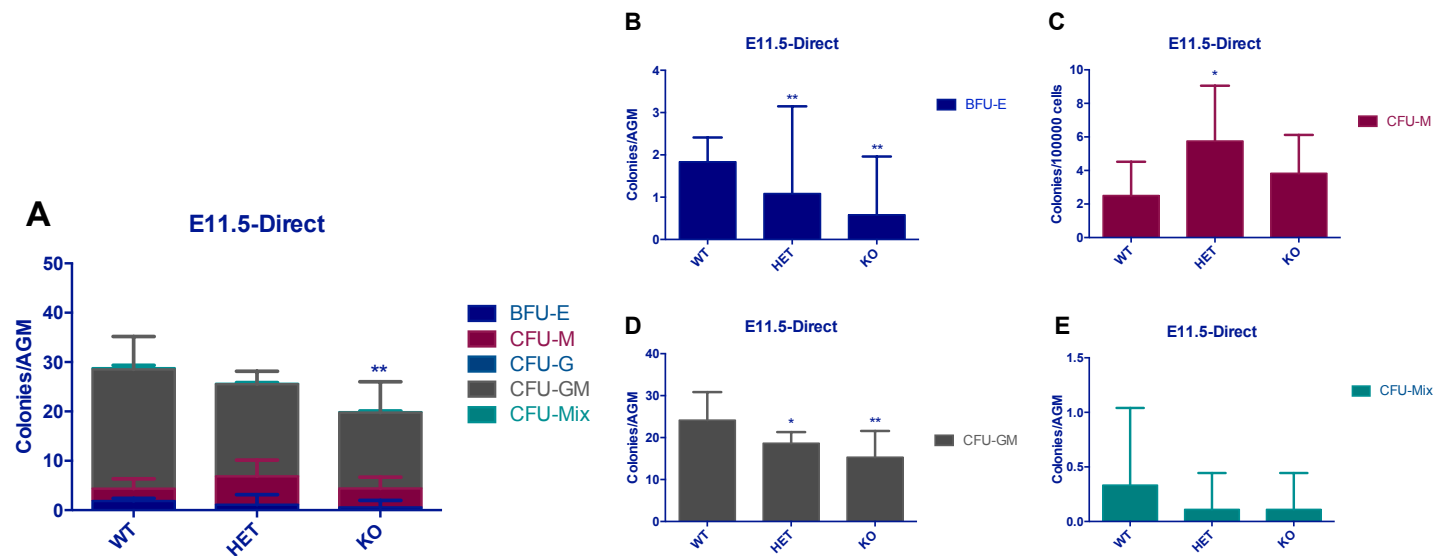

Figure 4-2: Bar graph shows total number of colonies and colony types obtained per $1 \times 10^{5}$ directly cultured E11.5 AGM cells. (A) Graph shows total number of colonies obtained per $1 \times 10^{5}$ directly cultured AGM cells. Bar graph shows numbers of BFU-E (B), CFU-M (C), CFU-GM (D) and CFU-Mix (E) colonies per $1 \times 10^{5}$ cultured AGM cells obtained for each genotype. Error bars indicate standard deviation. WT: wild-type $(\mathrm{G} 3+/+)$, HET: heterozygous (G3+/-), KO: knock-out (G3-/-). Statistical analysis were done using two-way ANOVA. $n=3-4$.

Specifically, this reduction was more noticeable in certain colony types: BFU-E and CFU-GM counts for the heterozygous embryos, although the colony count for the CFU-M showed a significant increase, whereas the reduction was significantly lower in the BFU-E and CFU-GM counts for the knockout embryos (Figure 4-2:B-E)

The same trend was observed in AGMs obtained from E10.5 embryos, albeit at a more significant level. In contrast to wild-type embryos, when one allele of Gata3 was deleted (G3+/-), the progenitor counts were significantly reduced by $40 \%$, and when both alleles were deleted, the reduction was more severe, reaching 64\% (Figure 4-3:A).

Colonies that were significantly affected were CFU-GM for the heterozygous, and CFU-M and CFU-GM for the knockout (Figure 4-3 B-E). 
A E10.5-Direct

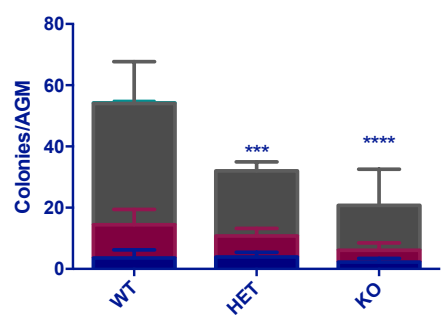

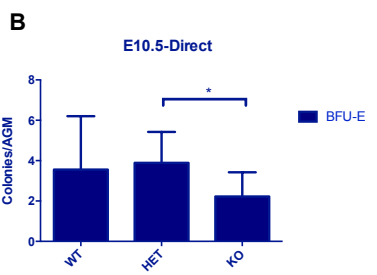
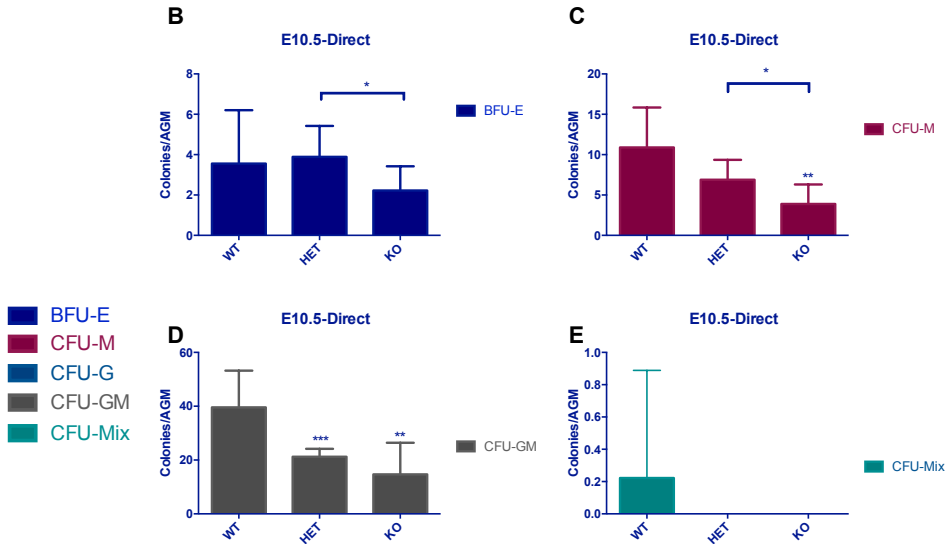

Figure 4-3: Bar graph shows total number of colonies and colony types obtained per $1 \times 10^{5}$ directly cultured E10.5 AGM cells. (A) Graph shows total number of colonies obtained per $1 \times 10^{5}$ directly cultured AGM cells. Bar graph shows numbers of BFU-E (B), CFU-M (C), CFU-GM (D) and CFU-Mix (E) colonies per $1 \times 10^{5}$ cultured $A G M$ cells obtained for each genotype. Error bars indicate standard deviation. WT: wild-type $(\mathrm{G} 3+/+)$, HET: heterozygous (G3+/-), KO: knock-out (G3-/-). Statistical analysis were done using two-way ANOVA. $n=3-4$

\section{4-4- G3+/- and G3-/- progenitors are unable to expand during explant culture:}

Given the migratory nature of haematopoietic cells, the direct evaluation of CFU-C content in the AGM may not give an accurate indication of their origin when measured directly, as a significant proportion will have arrived from the yolk sac via the circulation. Hence, an additional explant culture step was added to enumerate AGM-born progenitor numbers.

This model utilises a gas/liquid interface created by placing the whole AGM on top of a membrane instead of being submerged in a culture medium, which maintains the whole tissue structure, and gives a modest in vivo expansion of CFU-Cs within the AGM (Taoudi and Medvinsky: 2007).

Therefore, we investigated the potential of AGMs from all three genotypes from both E11.5 and E10.5 to develop and expand CFU-Cs independently using an explant culture system followed by methylcellulose cultures as above. 
As expected, this additional explant step expanded the number of colonies obtained from wild-type AGMs (compare Figure 4-2:A with Figure 4-4:A). In addition, it heightened the difference between wild-type AGMs and heterozygous and knockout AGMs, as the reduction in progenitor counts was more significant and consistent between different genotypes after the explant step (Figure 4-4:A and Figure 4-5:A). In contrast to the wild-type control, the heterozygous embryos produced fewer colonies by noticeable 34\%, and the knockout embryos by $69 \%$ at E11.5 (Figure 4-4:A). There was also a significant reduction of $53 \%$ when the knockout embryos were compared to the heterozygous embryos. (Figure 4-4:A).

A
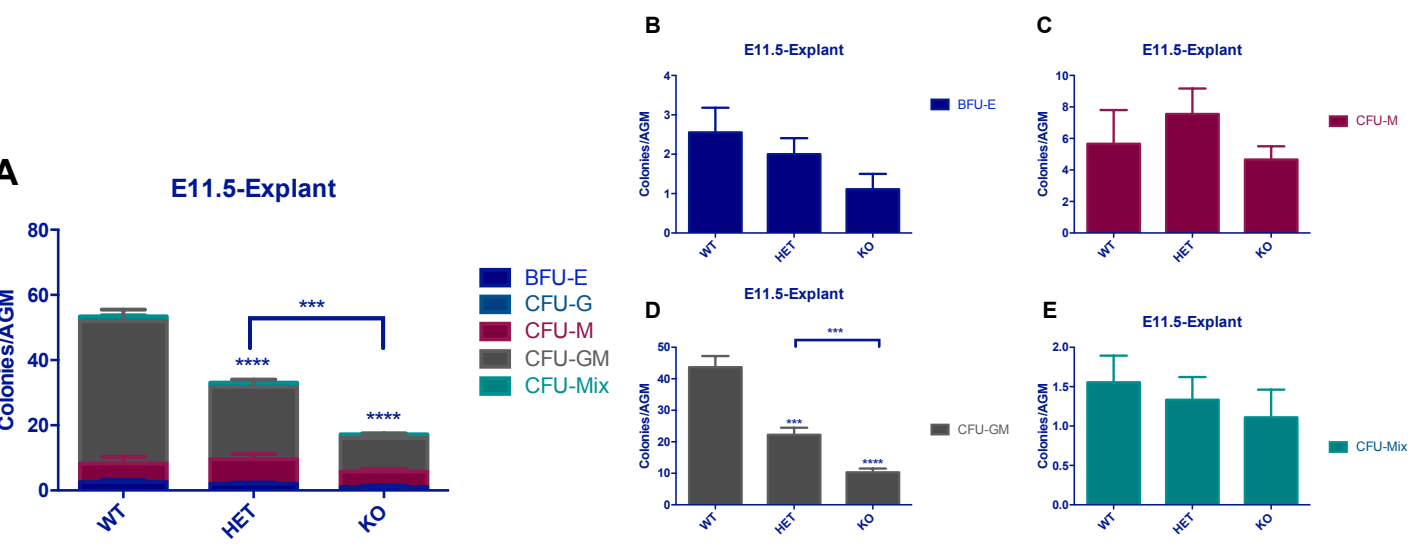

Figure 4-4: Bar graph shows total number of colonies and colony types obtained per $1 \times 10^{5}$ cultured E11.5 AGM cells after an explant step. (A) Graph shows total number of colonies obtained per $1 \times 10^{5}$ cultured AGM cells. Bar graph shows numbers of BFU-E (B), CFU-M (C), CFU-GM (D) and CFU-Mix (E) colonies per $1 \times 10^{5}$ cultured AGM cells obtained for each genotype. Error bars indicate standard deviation. WT: wild-type (G3+/+), HET: heterozygous (G3+/-), KO: knock-out (G3-/). Statistical analysis were done using two-way ANOVA $n=3-4$.

The colony types that were significantly affected by this were CFU-GM for both heterozygous embryos and knockout embryos by two and four fold reduction, respectively (Figure 4-4:B-E).

At E10.5, the reduction after the explant step in the heterozygous embryos was elevated to $74 \%$ (Figure 4-5:A) compared to $40 \%$ reduction without explant (Figure 4-3:A), while in the knockout embryos, the reduction 
observed was that of $90 \%$ compared to the wild-type AGM, and $60 \%$ lower than the heterozygous embryos.

This reduction was particularly noticeable in specific colony types for both genotypes: CFU-M and CFU-GM in comparison to the wild-type control. Between the G3+/- and G3-/-, there was a significant reduction in BFU-E, CFU-M, CFU-GM and CFU-Mix (Figure 4-5:B-E).

It thus appears that G3+/- and G3-/- progenitors are unable to expand under these conditions.
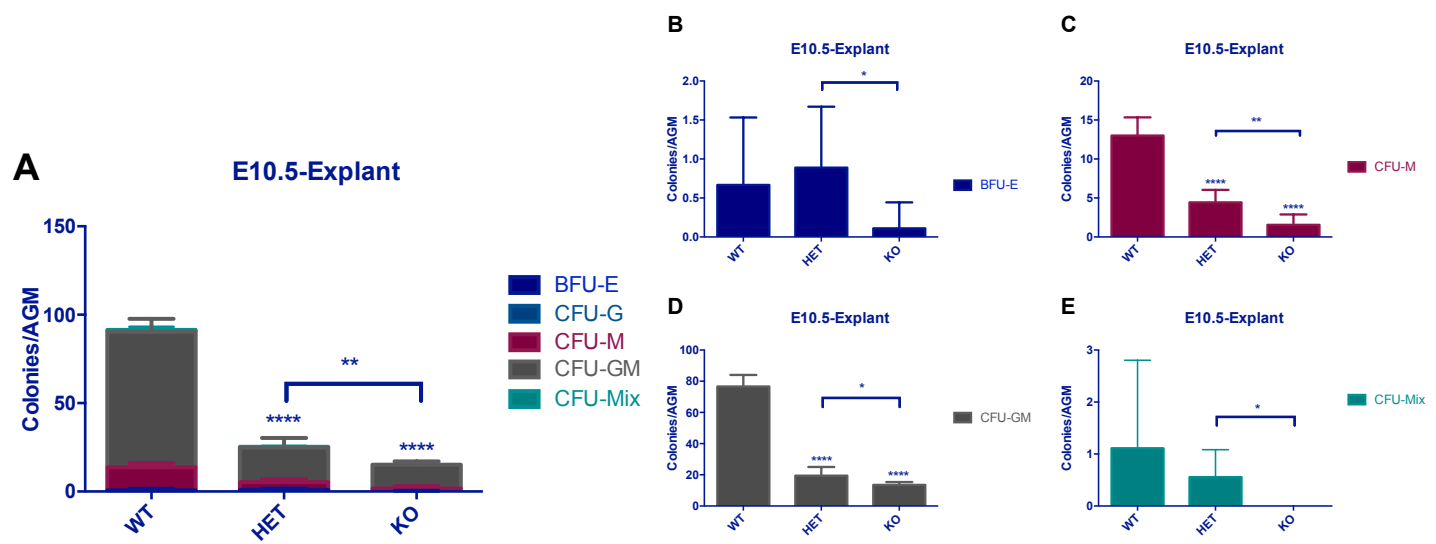

Figure 4-5: Bar graph shows total number of colonies and colony types obtained per $1 \times 10^{5}$ cultured E10.5 AGM cells after an explant step. (A) Graph shows total number of colonies obtained per $1 \times 10^{5}$ directly cultured AGM cells. Bar graph shows numbers of BFU-E (B), CFU-M (C), CFU-GM (D) and CFU-Mix (E) colonies per $1 \times 105$ cultured AGM cells obtained for each genotype. Error bars indicate standard deviation. WT: wild-type (G3+/+), HET: heterozygous (G3+/-), KO: knock-out (G3/-). Statistical analysis were done using two-way ANOVA. $n=3-4$.

\section{4-5- Gata3 within the HE plays a major role in haematopoietic} progenitor formation:

To gain further insight into the role of Gata3 in progenitor formation, we looked at the effect of Gata3 deletion specifically from the vascular endothelium on haematopoietic progenitor counts using the Vec-Cre system (Chen: 2009). By crossing conG3 f/f (Zhu: 2004) with conG3 f/t:+/cre we generated three genotypes: Gata3 f/f:+/cre (f/f:+/cre), which represents Gata3 deletion from the endothelium; Gata3 ${ }^{\mathrm{f} /+:+/ c r e}$ (f/+:+//cre), which represents a partial deletion or a 
heterozygous; and Gata3 f/f:+/+ or Gata $3^{f /+}:+/+$ ( f/f or f/+), which is the control and our equivalent to a wild-type in this experiment.

E10.5 and E11.5 AGMs of these genotypes were obtained and were either directly plated into methylcellulose for CFU-Cs, or explant cultured and then plated into methylcellulose for CFU-Cs (Figure 4-6).

Strikingly, the results were similar to those obtained from the global Gata3 deletions (4-2). With AGMs obtained from E11.5 embryos and directly plated in methylcellulose, the heterozygous AGM progenitor counts were $44.7 \%$ lower than those of the control, whereas the Gata3 f/f:+/cre were $52.6 \%$ lower than those of the control (Figure 4-7A).

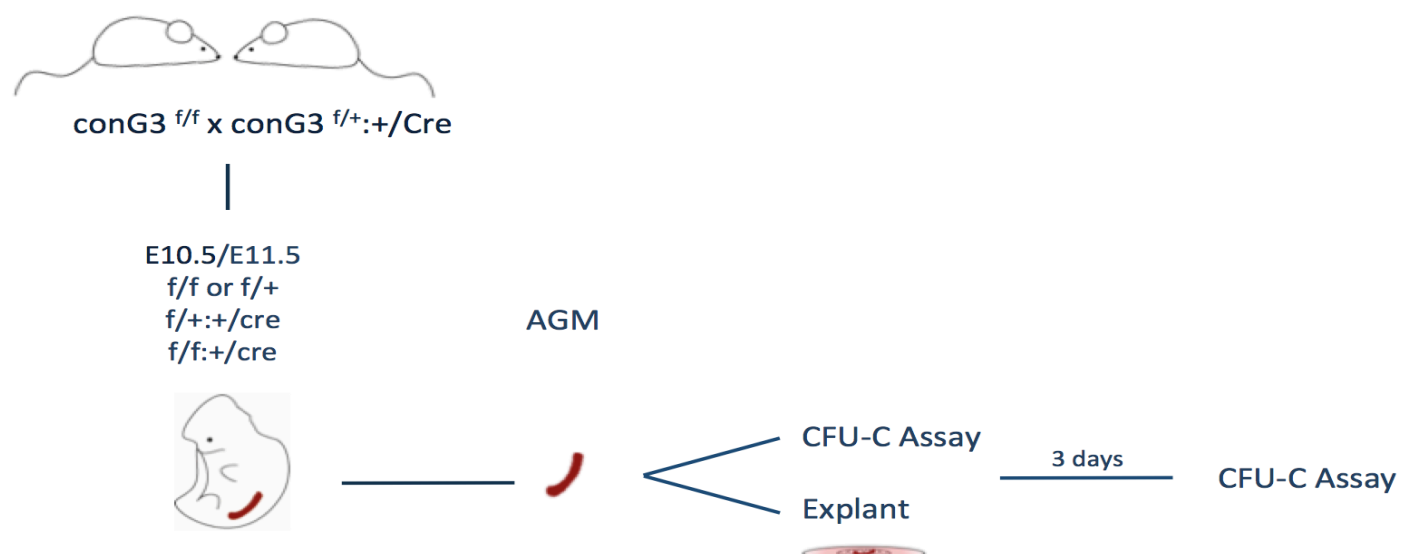

Figure 4-6: Representative schematic of the experimental design of CFU-C assay. AGMs are dissected from E10.5 or E11.5 f/f:+/+, f/+:+/cre, and f/f:+/cre embryos, and either dissociated and plated into methylcellulose medium directly, or cultured at the air/liquid interface for three days, harvested, dissociated and plated into methylcellulose medium.

The colonies that were mostly affected by Gata3 deletion from the endothelium were CFU-M, CFU-GM and CFU-Mix in both Gata3 f/+:+/cre and Gata3 f/f:+/cre (Figure 4-7 B-E). BFU-E showed an expected gradual reduction in colony counts with the highest numbers being in the control, followed by Gata3 $\mathrm{f} /+:+/ \mathrm{cre}$, and at last Gata3 f/f:+/cre where there were no colonies (Figure 4-7 B). 
CFU-GM had a somewhat different count; the control genotype had the highest colony count, and both Gata3 f/+:+/cre and Gata3 f/f:+/cre had similar colony counts (Figure 4-7 D).

However, in CFU-M there was a significant increase in both Gata3 f/+:+/cre and Gata3 f/f:+/cre with Gata3 f/+:+/cre having the highest colony count, followed by Gata3 f/f:+/cre and at last Gata3 f/f or f/+ (Figure 4-7 C). Furthermore, in the CFU-Mix, the highest colony count was that of the control, followed by the knockout (Gata3 f/f:+/cre), and the lowest count was that of Gata3 f/+:+/cre (Figure 4-7 E).
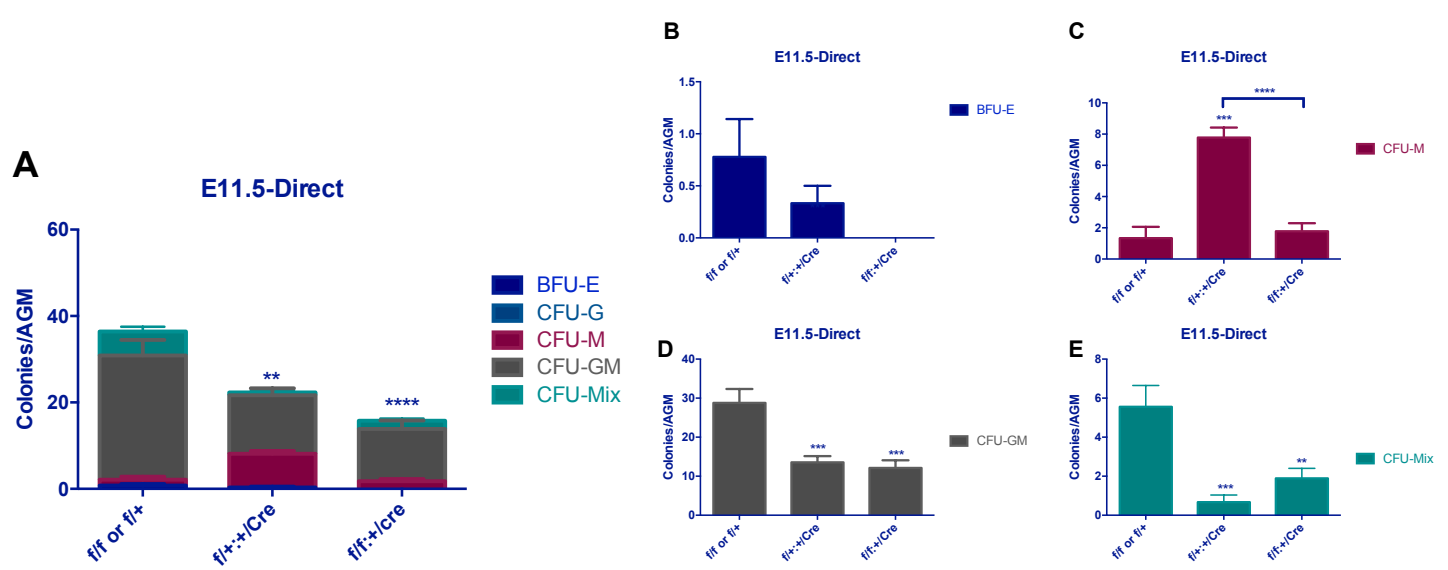

Figure 4-7: Bar graph shows total number of colonies and colony types obtained per $1 \times 10^{5}$ directly cultured E11.5 AGM cells. (A) Graph shows total number of colonies obtained per $1 \times 10^{5}$ directly cultured AGM cells. Bar graph shows numbers of BFU-E (B), CFU-M (C), CFU-GM (D) and CFU-Mix (E) colonies per $1 \times 10^{5}$ cultured AGM cells obtained for each genotype: $f / f$ or $f /+, f /+:+/$ cre, and $f / f:+/$ cre.. Statistical analysis were done using two-way ANOVA. $n=3-4$

Then we directly plated E10.5 AGM cells, and, similar to those in the global knockout, there was a reduction in both Gata3 f/+:+/cre and Gata3 $f / f:+/$ cre progenitor numbers, which was $70 \%$ and $67 \%$ lower than the control numbers, respectively (Figure 4-8 A-E). 

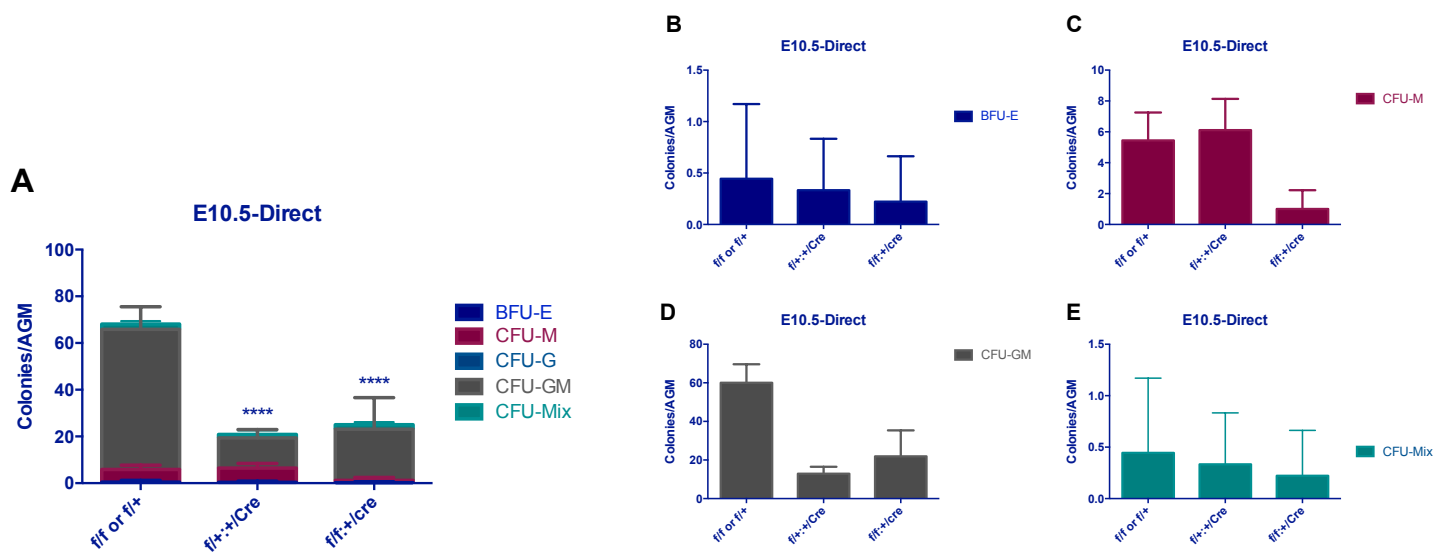

Figure 4-8: Bar graph shows total number of colonies and colony types obtained per $1 \times 10^{5}$ directly cultured E10.5 AGM cells. (A) Graph shows total number of colonies obtained per $1 \times 10^{5}$ directly cultured AGM cells. Bar graph shows numbers of BFU-E (B), CFU-M (C), CFU-GM (D) and CFU-Mix (E) colonies per $1 \times 10^{5}$ cultured AGM cells obtained for each genotype: $f / f$ or $f /+, f /+:+/ c r e$, and $f / f:+/ c r e$. Statistical analysis were done using two-way ANOVA. $n=3-4$.

Finally, we examined both stages after explant culture, given that it reflects the actual progenitor counts. At E11.5, progenitor numbers were similar to those following global Gata3 deletion and direct CFU-C assays from the conditionally deleted embryos, with Gata3 f/+:+/cre and Gata3 f/f:+/cre numbers $35.5 \%$ and 60\% lower than the control, respectively (Figure 4-9 A). Moreover, it also emphasized the difference between the different genotypes. The colonies that were mostly affected were CFU-M, CFU-GM and CFU-Mix in both Gata3 f/+:+/cre and Gata3 f/f:+/cre (Figure 4-9 B-E). While there was a reduction in BFU-E, CFU-M and CFU-GM, the CFU-Mix on the other hand showed a significant increase in Gata3 f/+:+/cre compared to Gata3 f/+ or Gata3 f/f and Gata3 f/f:+/cre genotypes. 

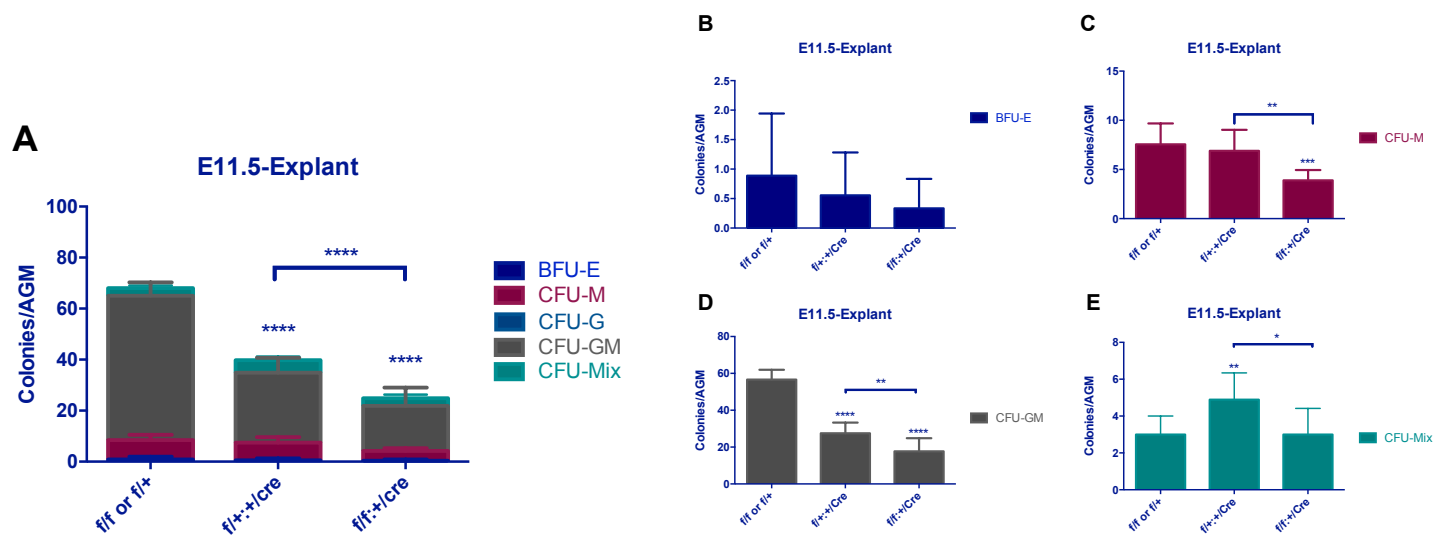

Figure 4-9: Bar graph shows total number of colonies and colony types obtained per $1 \times 10^{5}$ cultured E11.5 AGM cells after an explant step. (A) Graph shows total number of colonies obtained per $1 \times 10^{5}$ directly cultured AGM cells. Bar graph shows numbers of BFU-E (B), CFU-M (C), CFU-GM (D) and CFU-Mix (E) colonies per $1 \times 10^{5}$ cultured AGM cells obtained for each genotype: $f / f$ or $f /+, f /+:+/ c r e$, and $f / f$ : $+/$ cre. Statistical analysis were done using two-way ANOVA. $n=3-4$.

When the explant culture was performed with E10.5 AGMs, followed by CFU-Cs, the results were also similar to those of global knockout and direct CFUCs. The Gata3 f/+:t/cre colony count was 33\% lower than the control, whereas Gata3 f/f:+/cre was 63\% lower (Figure 4-10 A). The colonies that were mostly affected were CFU-mix in both genotypes (Figure 4-10 B-E).
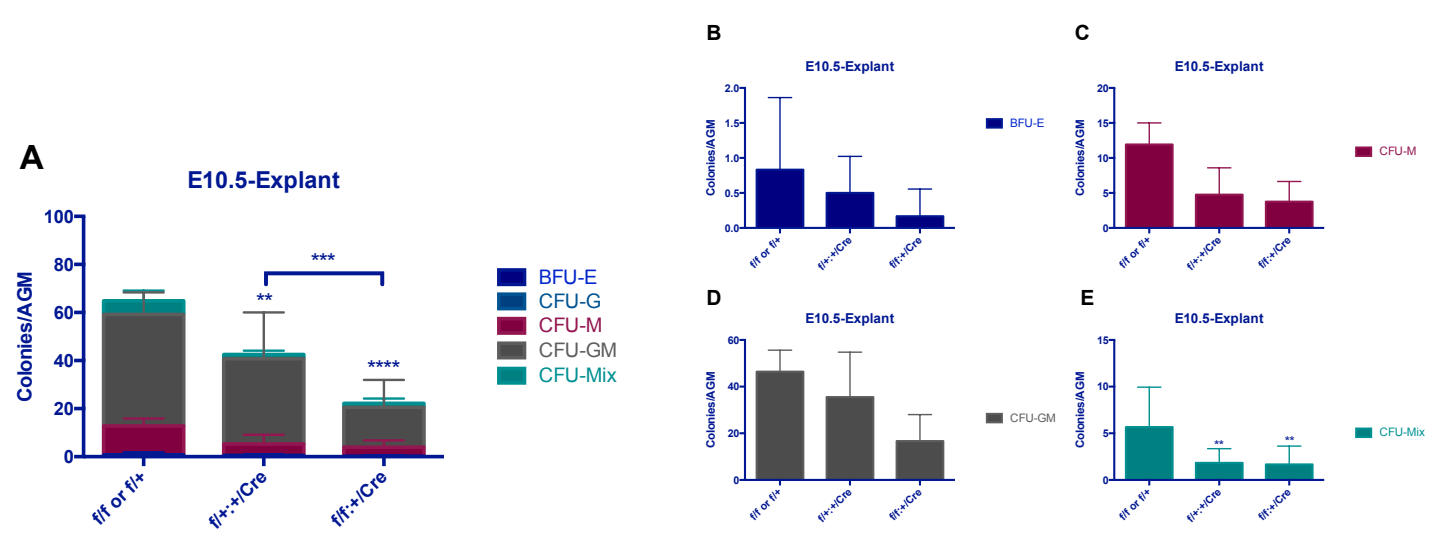

Figure 4-10: (A) Bar graph shows total number of colonies and colony types obtained per $1 \times 10^{5}$ cultured E10.5 AGM cells after an explant step. Bar graph shows numbers of BFU-E (B), CFU-M (C), CFU-GM (D) and CFU-Mix (E) colonies per $1 \times 10^{5}$ cultured AGM cells obtained for each genotype: $f / f$ or $f /+, f /+:+/$ cre, and $f / f:+/$ cre. Statistical analysis were done using two-way ANOVA. $n=3-4$. 
To confirm Cre-mediated Gata3 deletion, we performed PCR to determine the genotypes of the knockout colonies by picking them from the E10.5 Gata3 $f / f:+$ /cre plates at the end of the CFU-C assay. 20 CFU-GM colonies were picked and out of those colonies, 6 colonies seemed to have escaped Gata3 deletion, suggesting that the effect of endothelial-specific Gata3 deletion is even more severe (Figure 4-11).

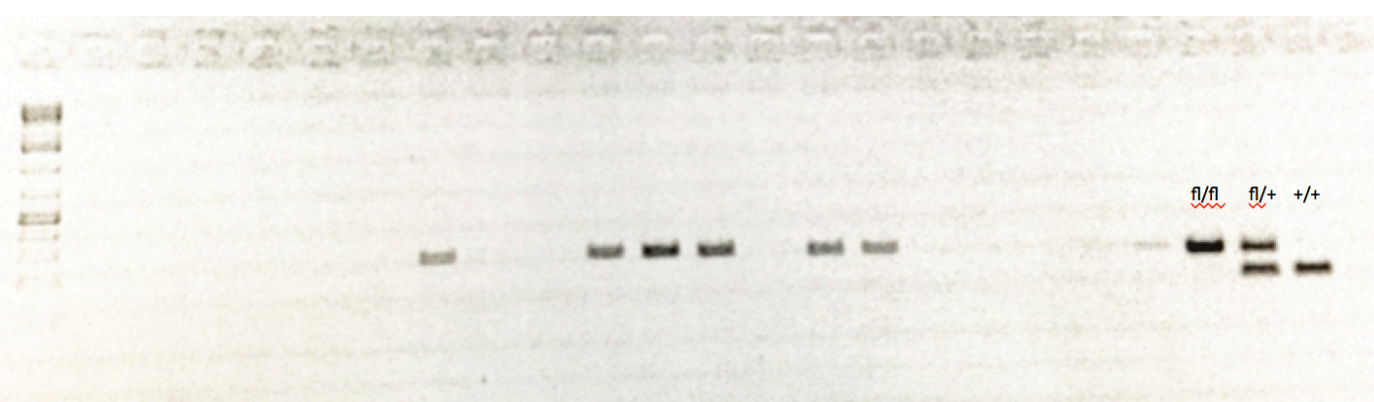

Figure 4-11: PCR for colonies picked from CFU-C assays derived from E10.5 explant cultured AGM cells. Six colonies out of 20 (25\%) have escaped the deletion.

\section{4-6- The effect of Gata3 deletion from endothelium on} haematopoietic repopulation:

In order to investigate the effect of Gata3 conditional deletion on haematopoietic repopulation and HSCs, we performed a transplantation assay. AGMs from E11.5 embryos of the various genotypes were transplanted into irradiated mouse recipients, and the repopulation was examined 4 months after the transplants (Figure 4-12).

These in vivo repopulation assays revealed that E11.5 AGMs of Gata3 $f / f:+/$ cre can repopulate irradiated recipients, but at a much lower rate, with only 1 mouse repopulated out of 12 transplanted (8.5\%) in contrast to the wild-type control where 5 out of 13 mice repopulated (38.5\%), and to Gata3 f/+:t/cre where 5 out of 10 recipients engrafted (50\%) (Figure 4-13). 


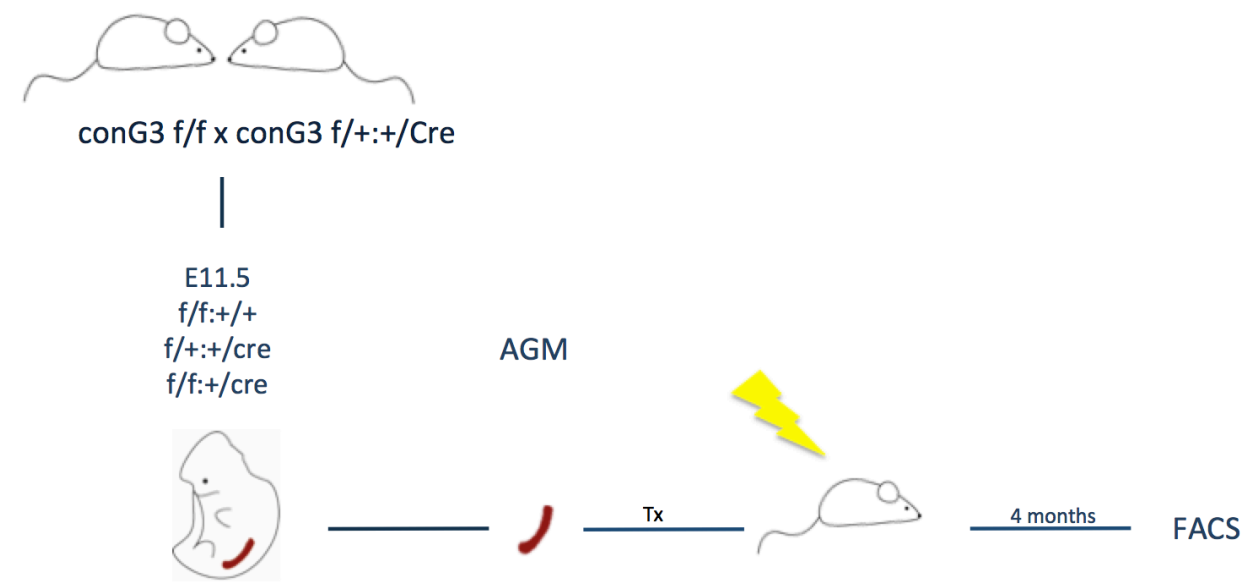

Figure 4-12: Representative schematic of the experimental design of the transplantation assays. AGMs are dissected from E11.5 embryos with the following genotypes: $f / f$ or $f /+, f /+:+/ c r e$, and $f / f:+/ c r e$ and transplanted into irradiated recipients. Mice are then bleed at 4 months and the donor cell contribution to the peripheral blood of the recipient is measured by flow cytometry analysis. Chimerism of 5\% or higher was set as the threshold at which mice are considered positive for repopulation.

\section{4 months}

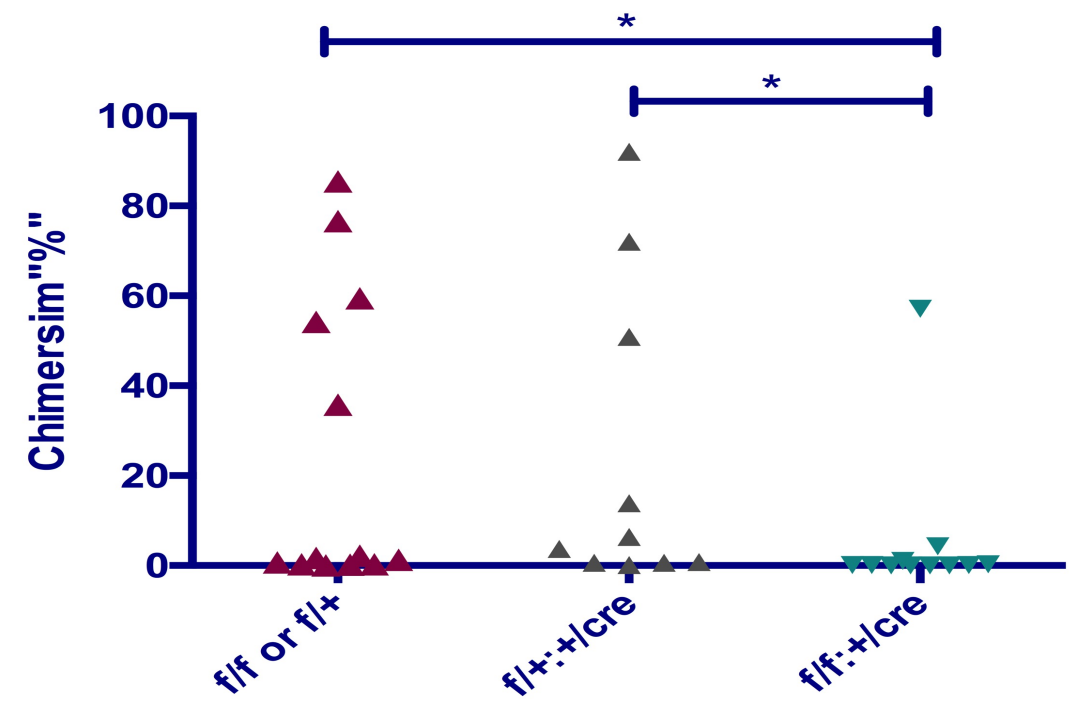

Figure 4-13: Levels of chimerism from transplanted AGMs of the following genotypes: $f / f$ or $f /+, f /+:+/ c r e$, and $f / f:+/ c r e .1$ ee was injected per mouse. Statistics were performed on GraphPad Prism and the Mann-Whitney test was used to determine significance levels. Chimerism of 5\% or higher was set as the threshold at which mice are considered positive for repopulation. $f / f$ or $f /+n=13, f /+:+$ cre $n=10, f / f:+$ cre $n=11$ 


\section{4-7- Discussion:}

HSCs and haematopoietic progenitors emerge from the vascular endothelium within the AGM (Jaffredo: 1998; Bertrand: 2010; Chen: 2009; de Bruijn: 2002; Kissa and Herbomel: 2010; Zovein: 2008). Our results demonstrate here that Gata3 in the endothelium is necessary for haematopoietic progenitor formation, and that it plays a role in HSC generation.

Previously, it was reported by the Ottersbach group that Gata3 deletion causes a significant reduction in HSCs and progenitor cells (CFU-S) in E11.5 and E10.5 AGMs, using a germline-deleted Gata3 knockout line. Here, we have demonstrated that Gata3 is also important for maintaining normal counts of other, more mature, haematopoietic progenitor cell, as enumerated in CFU-C assays. In fact, Gata3 reduction lowered haematopoietic progenitor cell numbers in both heterozygous and knockout embryos, which corresponds with what was reported before by Fitch et al (2012) of Gata3 haploinsufficiency reducing HSC activity.

Our findings here have revealed that the effect of Gata3 on progenitors is both dose-dependent and stage-dependent: i.e. haploinsufficient vs. knockout and E10.5 vs. E11.5. The reduction in the CFU-C counts seems to follow the same pattern at both E11.5 and E10.5; however, it is much more severe and pronounced at E10.5. Given the variation in the counts between embryos, and considering the migratory nature of haematopoietic cells, we added an explantculture step to ensure that these progenitors originated from the AGM (Medvinsky and Dzierzak: 1996; Kumaravelu: 2002; Taoudi: 2005; de Bruijn 2000). Interestingly, adding the explant step not only reduced the variation between embryos, reflecting a more accurate representation of the total number of progenitors in each AGM, but also revealed that Gata3+/- and Gata3-/progenitors are unable to expand during the explant culture, unlike wild-type progenitors. The reduction in both heterozygous and knockout AGMs compared to the wild-type control after the explant was elevated from $22 \%$ to $34 \%$ in E11.5 heterozygous AGMs, and 56\% to 69\% in knockout AGMs. The reduction in CFU-Cs of E10.5 AGMs was from $40 \%$ to $74 \%$ in heterozygous, and from $64 \%$ to 
$90 \%$ in knockout embryos. This reduction following explant culture could be attributed to Gata3 involvement in regulating cell cycle entry, which corresponds with what was reported for HSCs by Ku et al. (2007).

It should be noted here that among the colonies, CFU-M seemed to be the least affected by Gata3 reduction and/or absence. This could contributed to the fact that these colonies are produced by the EMPs, which are produced in the yolk sac during the primitive haematopoiesis, a process that has shown to be Gata3 independent (Pandolfi: 1995).

The fact that the reduction at E10.5 was more pronounced than at E11.5 could be explained by the presence of a transient Gata3+ haematopoietic cell population that appears around E10 and disappears by E11.5. This population was identified during the Gata3 expression analysis (section 3-3-2), and the absence of one or both alleles of Gata3 may have affected that population.

Another explanation for this could also be the involvement of Gata3 in HE function, and that its absence or haploinsufficiency could impair its haematopoietic potential, and resulting in lower output of haematopoietic progenitors from endothelial precursors.

The latter interpretation receives support from the experiments with the conditional Gata3 knockout line. When Gata3 was conditionally deleted from the vascular endothelium, it caused a significant drop in progenitor counts in both Gata3 f/+:+/cre and Gata3 f/f:+/cre. Similar to the global deletion of Gata3, the effect was more pronounced at E10.5 and even more so after adding an explant culture step. This demonstrates that Gata3 is essential in the endothelium for haematopoietic progenitor formation.

Gata3 has been previously implicated in cell cycle regulation (Ku: 2012). When Gata3 was deleted; fewer HSCs were produced, they were more quiescent, and failed to enter the cell cycle after myelosupression to recover. This could explain the more pronounced effect on CFU-C counts after the additional explant step, during which progenitors are thought to undergo an expansion. 
Gata3 in the vascular endothelium also plays a role in HSC generation. Transplantation assays have revealed here that when Gata3 was deleted from the endothelium, it almost completely abrogated engraftment potential in AGM cells. Some residual HSC activity remained in Gata3-null AGMs, which may be due to some haemogenic endothelial cells having escaped Cre-mediated deletion. However, previous transplant experiments with germline-deleted Gata3 knockout AGMs have also detected some, albeit greatly reduced, repopulating activity (Fitch 2012), which may indicate that Gata3 is important, but not essential, for EHT. 


\section{5- Gata3 expression in the HSC microenvironment}

\section{5-1- Background:}

Gata3 is expressed in various cell types in the AGM, and our previous findings (chapter 3) have shown that expression to be stage dependent. It is expressed in sympathoadrenal cells (Fitch: 2012), mesenchymal cells (Manaia: 2000), endothelial cells (Manaia: 2000; Swiers: 2013), and in haematopoietic cells (Swiers: 2013), all of which we confirmed in chapter 3.

Previously, we have confirmed the expression of Gata3 in a subset of each of those 4 populations, and performed functional assays to confirm a role of Gata3 in the haematopoietic and endothelial populations. However, how these four populations interact with one another and how they affect HSC emergence is still to be investigated. Furthermore, how the Gata3-expressing subpopulation differs from the Gata3-negative one has not been fully investigated for all of these.

Gata3 has been implicated in cell cycle regulation. Previously, $\mathrm{Ku}$ et al. (2012) demonstrated that Gata3 is required for both promoting HSC cell cycle entry and maintaining normal numbers of adult HSCs. Gata3 involvement in cell cycle regulation could also explain the increased reduction in progenitor counts from Gata3-deficient AGMs after the explant culture step in the CFU-C assays in chapter 4.

Here, we use a highly sensitive RNA-seq method to examine the whole transcriptome of small numbers of Gata3-expressing subpopulations within the immediate environment of HSCs (HC, MC, SNS, and EC) and compared them to their non-Gata3 expressing counterparts. Given the limited number of Gata3 expressing cells these populations, RNA sequencing provides the most efficient and sensitive method for analysis of these cells. A possible link of Gata3 to cell cycle regulation will also be investigated in these data sets. 
5-2-Aims:

Identify key genes and processes that correlate with Gata3 expression in the HSC immediate microenvironment.

Investigate the effect of Gata3 expression on cell cycle in HSC immediate microenvironment 


\section{5-3- Gata3+ and Gata3- cells segregate into distinct clusters:}

To analyse gene expression in our identified populations form chapter 3; $\mathrm{G} 3+\mathrm{HC}, \mathrm{G} 3+\mathrm{EC}, \mathrm{G} 3+\mathrm{SNS}$ and $\mathrm{G} 3+\mathrm{MC}$, in parallel to non-Gata3 expressing counterparts; G3-HC, G3-EC, G3-SNS, and G3-MC, we dissected AGMs from G3GFP embryos of E10.5 for haematopoietic and endothelial populations, and E11.5 for mesenchymal and SNS populations since these stages reflect the peak of Gata3 expression in each population (Swiers: 2013; Manaia: 2000; Pandolfi: 1995; Fitch: 2012; and chapter 3 of this thesis). The cells were then sorted according to Figure 5-1, and the transcriptome of each population analyzed by RNA-Seq.

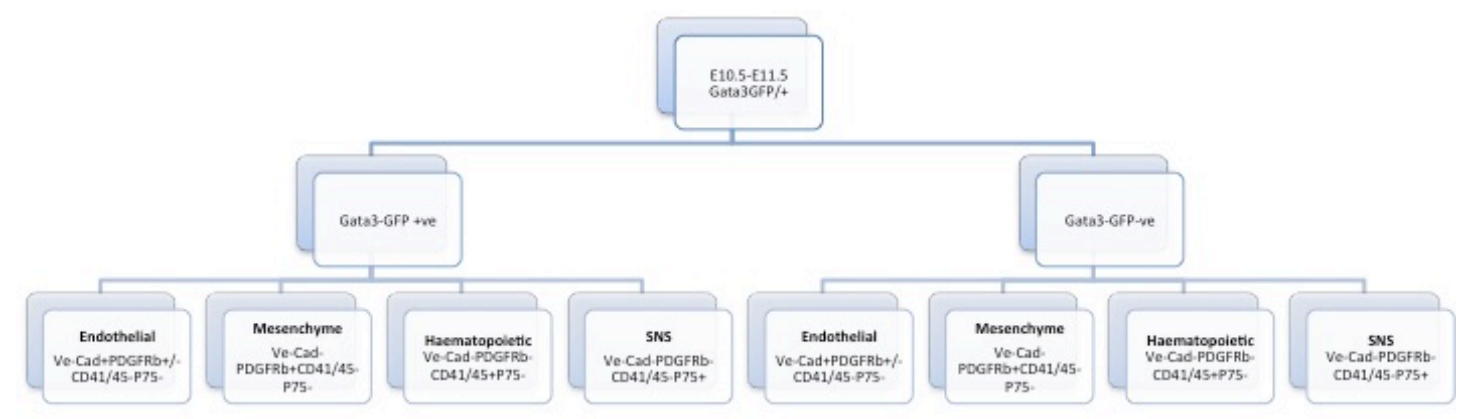

Figure 5-1: RNA-Sequencing sorting strategy. Cells are obtained from G3-GFP embryos, E10.5 for haematopoietic and endothelial cells, and E11.5 for SNS and mesenchymal cells. Cells were double sorted; the first sort was to isolate the populations in E10.5 G3+EC, G3-EC, G3+HC and G3-HC, and in E11.5 G3+SNS, G3SNS, G3+MC, and G3-MC. Each of these populations was then sorted again into a 96well plate into 20 wells; 20 cells/ well and a 400 cells were collected for each population. Cells were obtained from 3-5 independent biological replicates.

The principal component analysis (PCA) shows a clear separation between all eight populations. While cells of the same population cluster in the same region i.e. HC, EC, SNS and MC, each of the Gata3-expressing population nevertheless shows a clear distinct cluster from its non-Gata3 expressing population (Figure 5-2). 


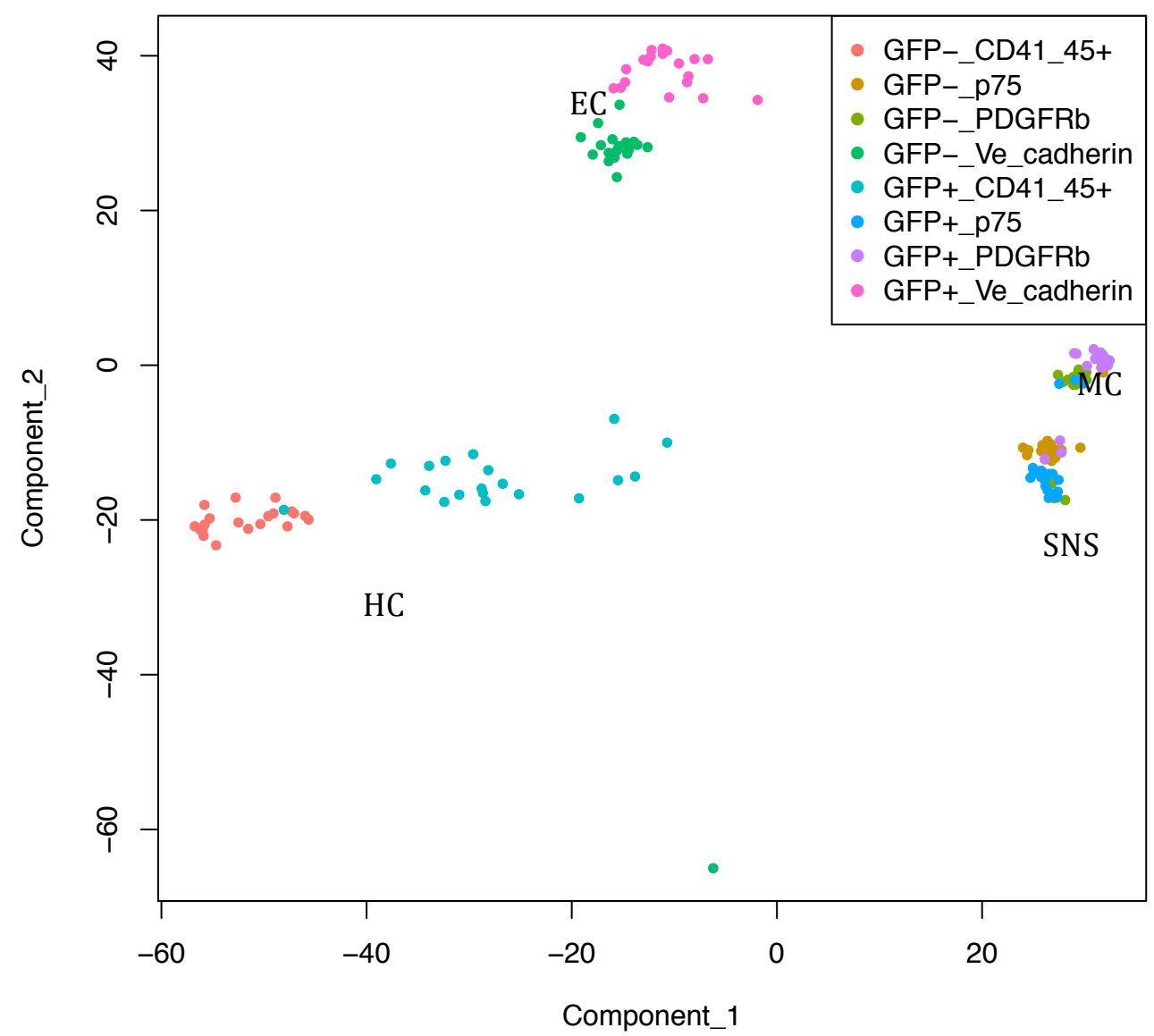

Figure 5-2: PCA showing the distribution of all Gata3-expressing populations G3+EC (GFP+Ve-Cad+), G3+HC (GFP+CD41/45+), G3+SNS (GFP+P75+), and G3+MC $(G F P+P D G F R b+)$ compared to non-G3 expressing cells of same populations: G3-EC (GFP-Ve-Cad+), G3-HC (GFP-CD41/45+), G3-SNS (GFP-P75+), and G3-MC (GFP$P D G F R b+) . n=400$ cells per population distributed equally over 20 wells.

Next we studied each population individually and compared the Gata3expressing subfraction to the Gata3- population. This again showed that each group formed a distinct cluster based on Gata3 expression or absence (Figure 53 A-D).

While all of the cell types show a clear separation between each of its subpopulations, the SNS seemed to have a transitional shape, which could be contributed to the on-going development of the SNS following the migration of 
neural crest cells to the neural patches in order to form the SNS with Gata3 marking later stages of maturation (Figure 5-3 B).

A

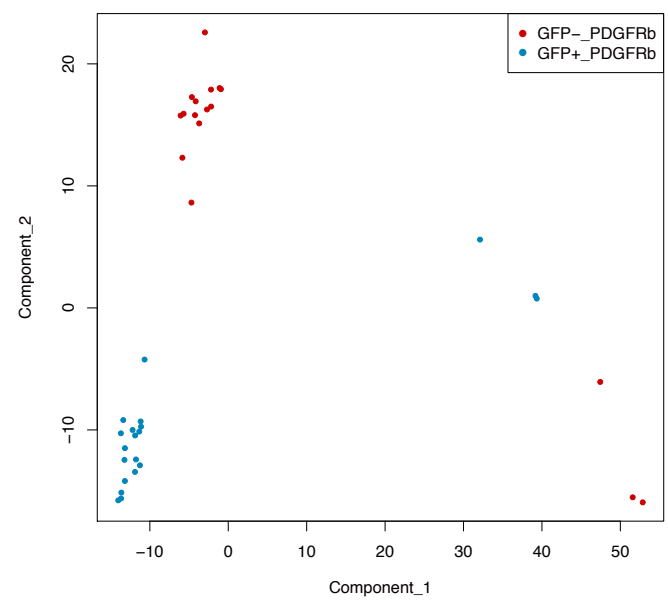

C

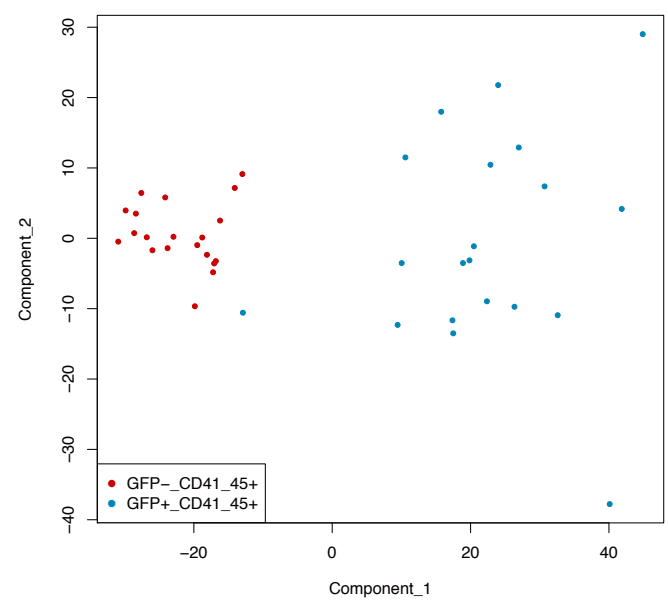

B

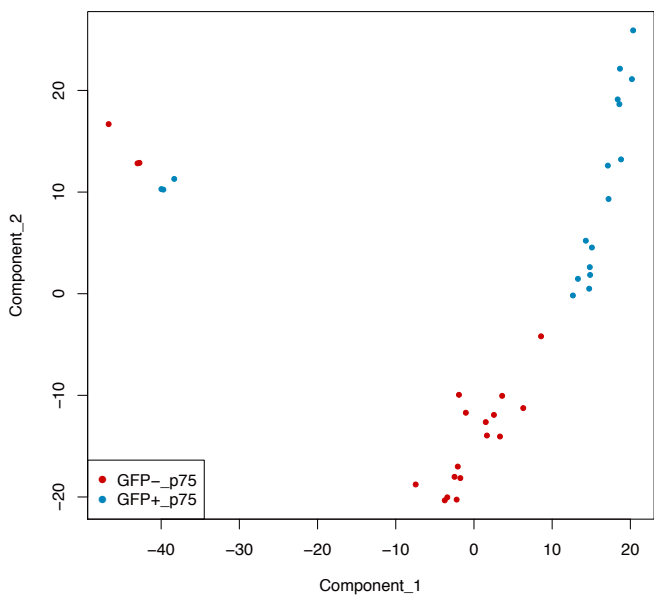

D

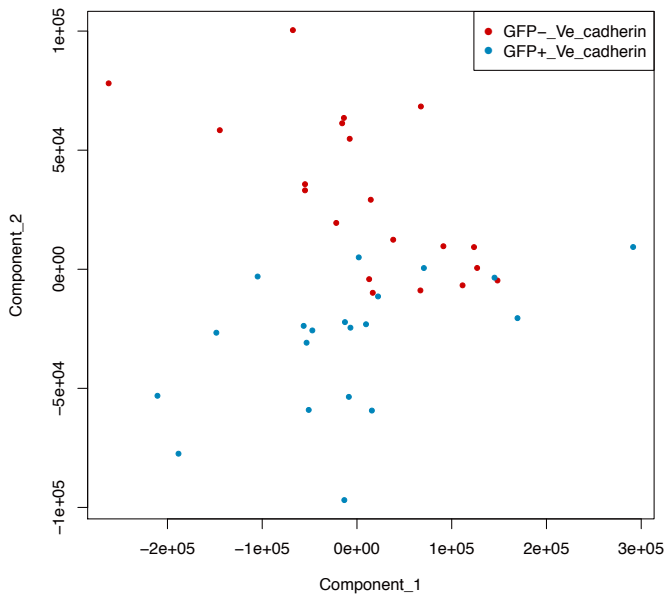

Figure 5-3: PCA showing the clustering of Gata3 expressing populations in contrast to their G3-counterpart. Individual panels represent each population: (A) G3+MC $(G F P+P D G F R b+)$ vs. G3-MC (GFP-PDGFRb+), (B) G3+SNS (GFP+P75+) vs. G3-SNS $(G F P-P 75+),(C) G 3+H C(G F P+C D 41 / 5+)$ vs. G3-HC (GFP-CD41/45+) and (D) G3+EC $(G F P+V e-C a d+)$ vs. G3-EC (GFP-Ve-Cad+). $n=400$ cells for each population distributed equally between 20 wells. 
Furthermore, endothelial cells seemed to be intermingled in the middle before they separate, which could be attributed to EHT taking place in some of these cells before forming haematopoietic cells, and then forming distinct clusters that separate endothelial cells from haemogenic endothelium, with Gata3 marking haemogenic endothelium (Figure 5-3 D).

Compared with the G3-populations, the expression of Gata3 results in the downregulation of 232 genes in the haematopoietic cells, 254 genes in the endothelial cells, 74 genes in the mesenchymal cells and 226 genes in the SNS cells (Figure 5-4 A). In contrast, the upregulated genes were much higher than the downregulated ones in the HC compartment (1528), in the ECs (558) and in the MCs (252). However, the upregulated genes in the SNS were slightly lower: 217 (Figure 5-4 A and B).

We next performed differential gene expression analysis of each of those populations in order to identify how Gata3 expression affects each population, and to identify the similarities and differences between these populations.

Within the downregulated genes, there was little overlap between the different cell populations, EC and HC overlapped in 7 genes, EC and MC had two genes in common, EC and SNS had only two genes in common, MC and HC had 2 genes in common, $\mathrm{HC}$ and SNS had also two genes in common between them, and SNS and MC which had one gene in common between them and EC as well (Figure 5-4 A).

The upregulated genes showed more overlap between the different populations: EC and HC had 160 genes in common, EC and MC had 45 genes in common, EC and SNS had 9 genes in common, MC and HC had 69 genes in common, HC and SNS had 46 genes in common, and similar to the downregulated genes, MC and SNS had one gene in common (Figure 5-4 B). 

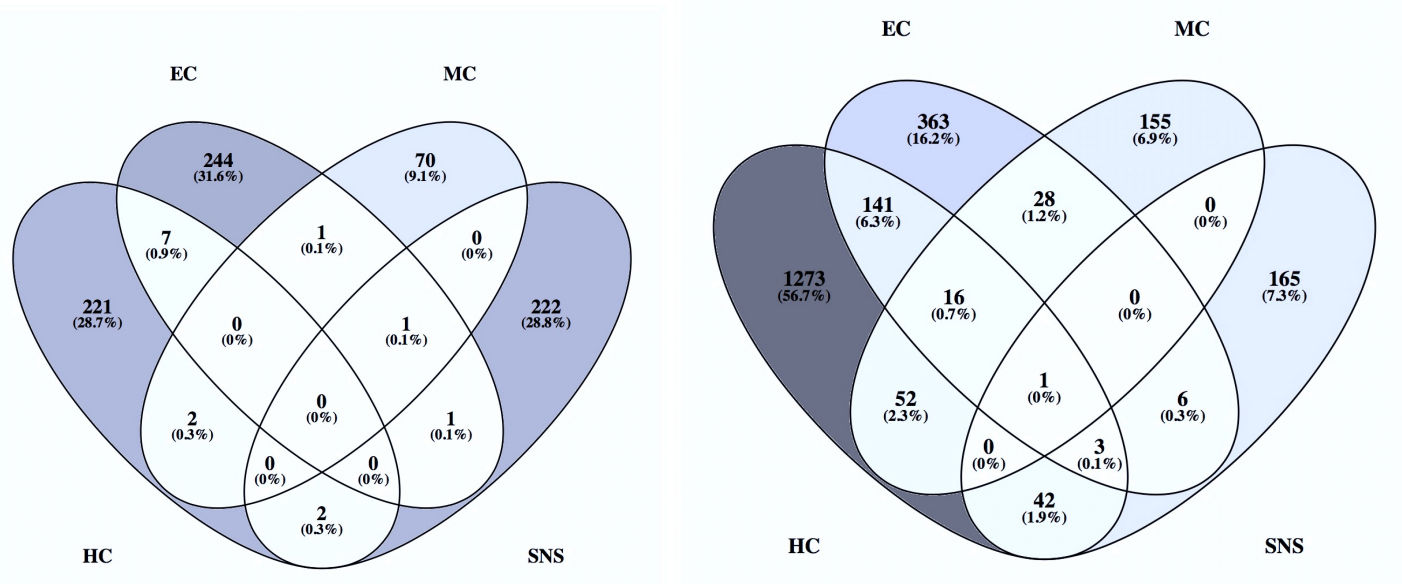

Figure 5-4: Venn diagrams showing the overlapping genes between the different Gata3-expressing populations where (A) represents the downregulated genes in Gata3-expressing populations and (B) represents the upregulated genes in Gata3expressing populations. EC: endothelial cells, MC: mesenchymal cells, HC: haematopoietic cells, and SNS: sympathetic nervous system. The differential gene analysis was done on DE-Seq using the adjusted $P$ value of 0.01 and $\log 2$ fold change of 0.1 .

\section{5-4- The downregulated genes are associated with development and} differentiation of various cells and systems:

To learn more about the differentially expressed genes and the biological processes they are involved in, we performed Gene Ontology (G0), first for the downregulated genes. Given the amount of genes that were identified, I have only selected the GO for biological processes that are relevant to this thesis in each population (Figure 5.5). 
$A$

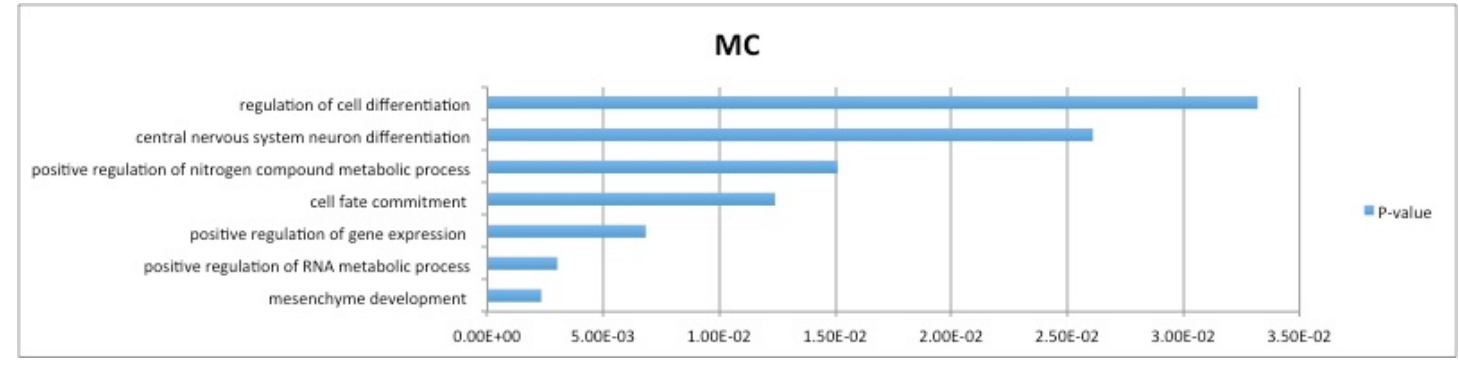

$B$

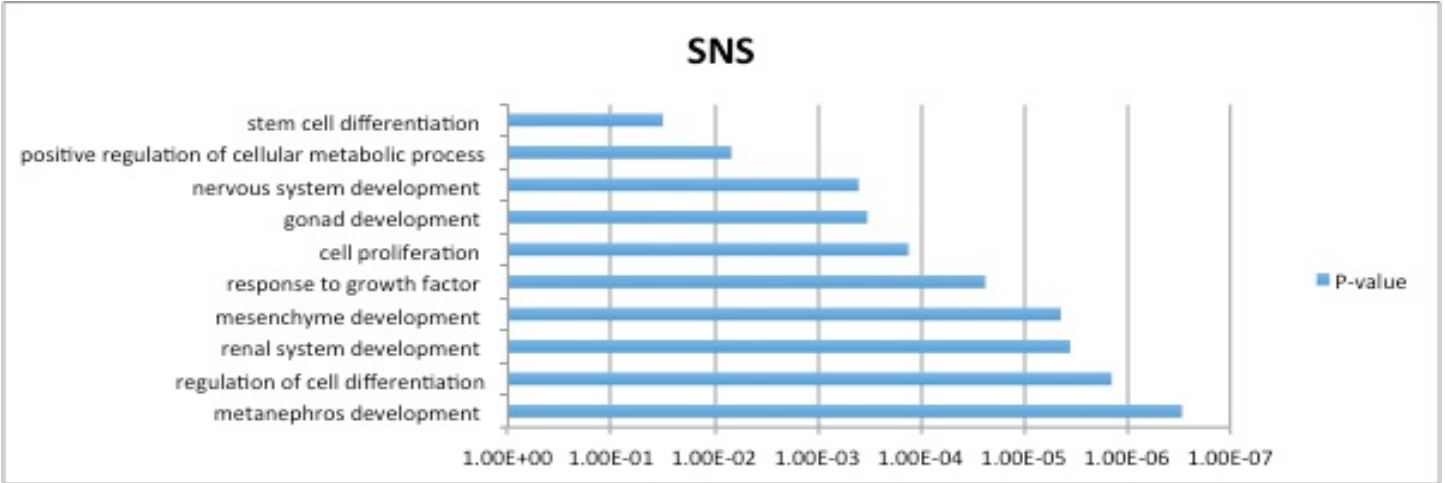

$C$

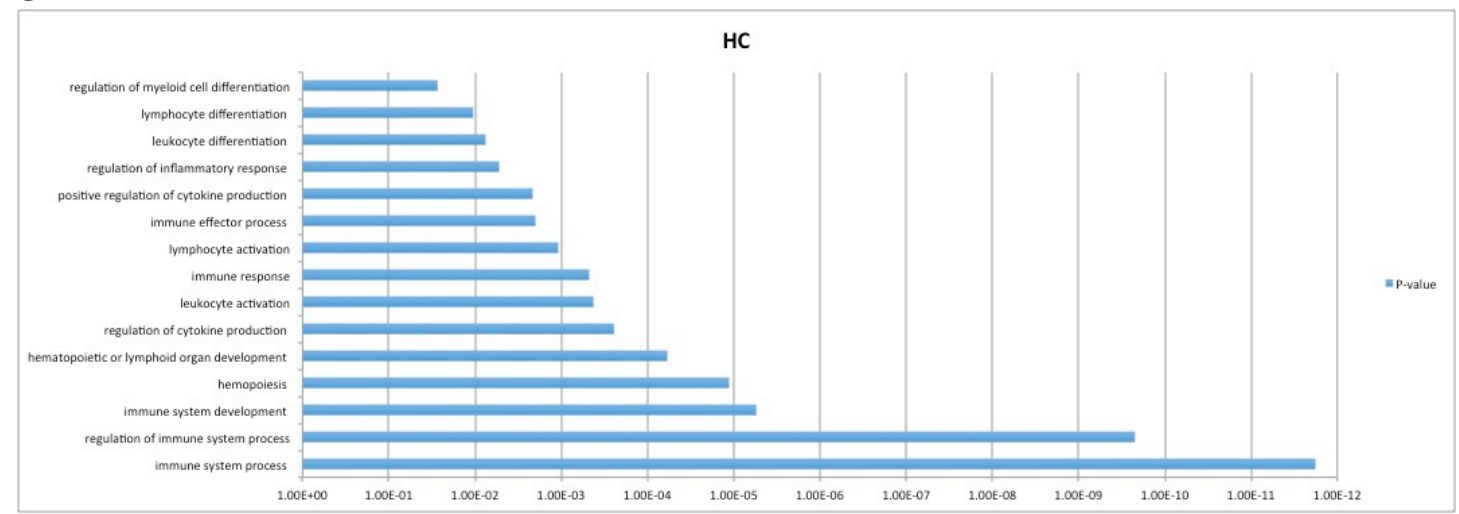

$D$

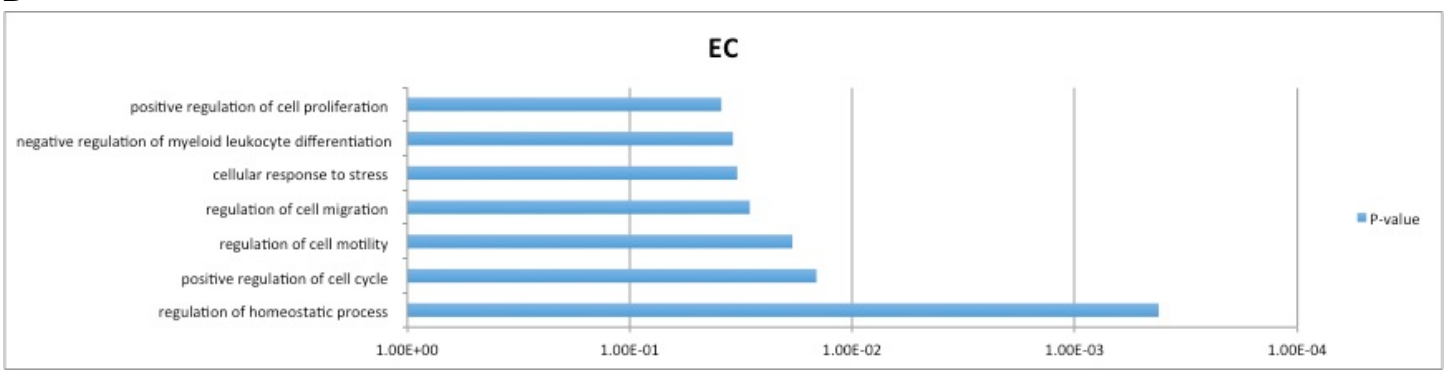

Figure 5-5: GO terms enriched in the downregulated genes in Gata3+ cells in contrast to Gata3- cells. (A) MC: mesenchymal cells, (B) SNS: Sympathetic nervous system, (C) HC: haematopoietic cells, and (D) EC: endothelial cells. . Gene enrichment for biological processes analysis was done on Gene Ontology Consortium website using the PANTHER classification system. 
Of note, most of the gene enrichments for biological processes in all populations were involved with development and differentiation of several cell types and tissues whether its central nervous system neuron development (Wnt2, Wnt5a, and Cbln1), and mesenchyme development (Cbln1, Ptch1, and Epha4) in MC compartment; or stem cell differentiation (Sfrp1, Ntf4, and Gdnf), nervous system development (Fgf20, Chl1, and Sdc4), gonad development (Dmrt1, Lhx9, Nr5a1, Kitlg, Gata4, and Bcl2), and mesenchyme development (Bmp7, Smad6, Gdnf, Has2 and Fgf10) in SNS compartment; myeloid cell, lymphocytes, and leukocytes development and activation (Nfam1, and Spi1), immune system development (Icos, Stap1, Myb, and Irf8), cytokine production (Casp1, Ccl3, Cybb, and Il6ra ), and haematopoiesis (Gpr183, Klf4, Cdk6, Stap1, and Anxa1) in HC; and myeloid and leukocytes differentiation in ECs in addition to the regulation of motility, cell cycle and homeostasis (Myc, Cd36, Mif, Gnl3, and Mafb) (Figure 5-5).

\section{5-5- The upregulated genes are associated with migration,} proliferation, development and apoptosis:

The upregulated genes were found to be mostly enriched for the following GO terms; development, migration, proliferation and apoptosis (Figure $5-6)$.

In the MC population, the gene enrichment was mostly for apoptosis regulation (Gadd45b), regulation of cell proliferation (Btc, Ctgf, Figf, and Pgf), adhesion (Fn1, Smad6, Cntn4, and Postn), differentiation (Gdnf, Fn1, Gata6, Frzb and Lama2), and migration (Fn1 and Angpt2), as well as development of the urogenital system (Lama5, Myocd, and Col4a1), muscle processes (Smad7, Dmpk, Dsg2 and Ctgf), circulatory system and angiogenesis (Hif3a, Figf, and Mcam) (Figure 5-6 A). 
A

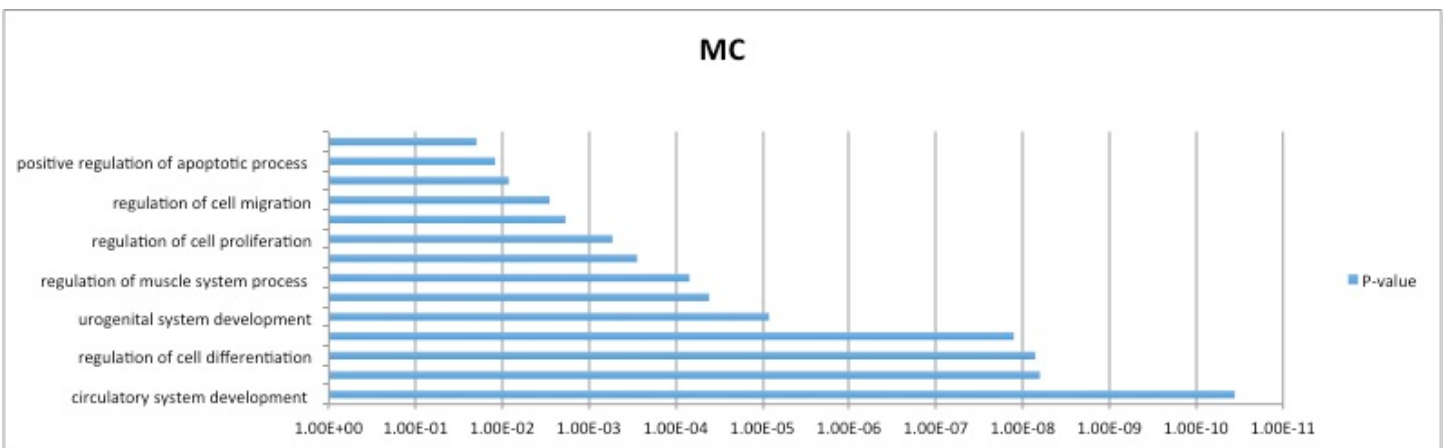

B

SNS

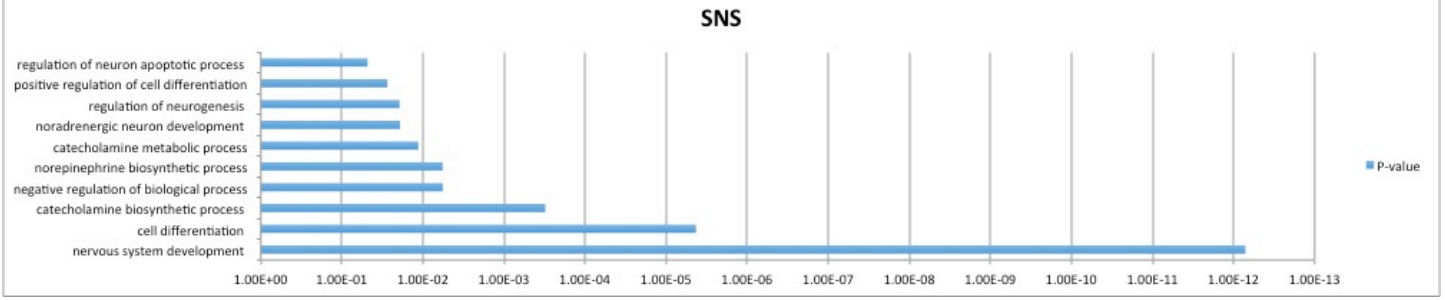

C

HC

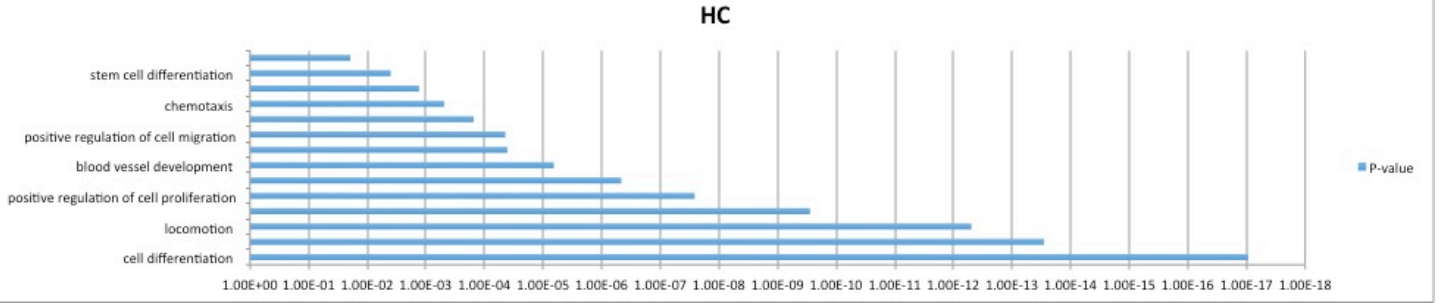

D

EC

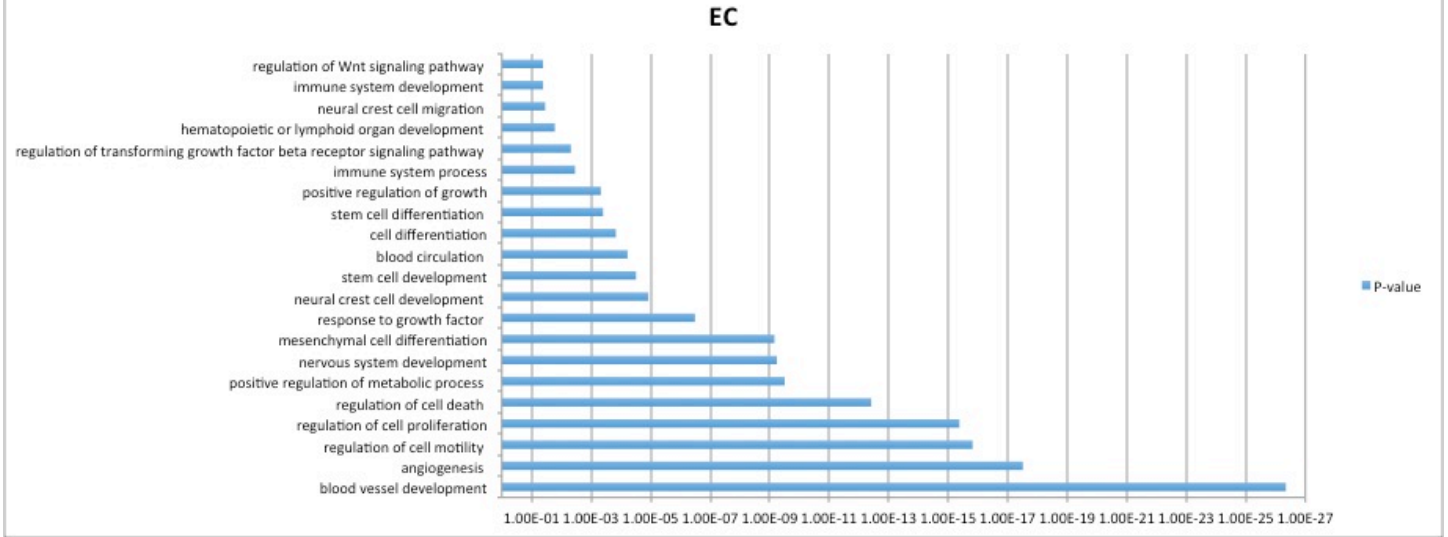

Figure 5-6: GO terms enriched in the upregulated genes in Gata3+ cells in contrast to Gata3- cells. (A) MC: mesenchymal cells, (B) SNS: Sympathetic nervous system, (C) HC: haematopoietic cells, and (D) EC: endothelial cells. +). Gene enrichment for biological processes analysis was done on Gene Ontology Consortium website using the PANTHER classification system. 
In the SNS, most of the gene enrichment was associated with the GO terms of cell differentiation (Sox7, Nefl, and Ngf), nervous system development (Map1b, CD9, and Tlx,), catecholamine metabolic and biosynthetic processes (Th, Gch1, and Agtr2), norepinephrine synthesis (Dbh, and Insm1), and neuron apoptotic process (Crlf1, Kcnip3, Ascl1, Agtr2, Mt3 and Sct) (Figure 5-6 B).

The HCs were mainly enriched for genes that are involved in chemotaxis, locomotion, cell migration, cell adhesion (Esam, Bcam, and Kitlg), stem cell and cell differentiation (Sox10, Sox11, Hand2, Isl1, Jag1, and Sox8), cell proliferation and blood vessel development (Mmp2, Fn1, Pdgfra, Lef1, Endra, Bmp4, Cdh5, Cdh13, Fgfr1, Hand1, Pecam1, and Angpt1) (Figure 5-6 C).

The gene enrichment in the ECs was associated with the development of cells and stem cells (Jag1, Nrp1, Fn1 and Lama5), blood vessels (Mmp2, Cdh5, Bmpr2, Smad7, and Cxcl12), haematopoietic or lymphoid organ (CD34, Mapk3, Jag2, Vegfa, and Sox4), nervous system (Bmpr2, Cxcl12, and Sox6), and immune system (Tek, Pbx1, and Gata2). It was also enriched for genes associated with cell motility (Fn1, Tie2, Cxcl16 and Bcr), cell proliferation (Pbx1, and Gata6), cell death (Fas, Pdcd4, and Notch1), cell differentiation (Meis2, Cdh5, Smad7, and Emp2), and blood circulation (Foxc2, Vegfc, Emp2 and Cav1) (Figure 5-6 D).

Of note, the Gata3+ ECs were enriched for genes that were involved in the regulation of transforming growth factor beta receptor signalling pathway and in the regulation of Wnt signalling pathway.

The overall picture of GO terms and differential gene expression, where cyclin dependent kinases (Cdk) were identified in the downregulated genes (HC and EC), and in the upregulated genes (HC, EC and SNS), suggested an involvement of Gata3 in cell cycle regulation. I therefore decided to further analyse the cell cycle status of Gata3-expressing populations compared with their Gata3- counterparts. Strikingly, we found only pro-proliferation Cdks in the downregulated genes, whereas it is almost only cell cycle inhibitors in the upregulated genes (Table 5-1). 


\begin{tabular}{|lll|}
\hline Cell Type & Upregulated Cdks & Downregulated Cdks \\
\hline HC & Cdkn1c & Cdk6 \\
& Cdkn2d & \\
\hline EC & Cdkn1c & Cdk1 \\
& Cdk19 & Cdkl1 \\
\hline SNS & Cdkn1c & - \\
\hline
\end{tabular}

Table 4: List of cyclin dependent kinases (Cdk) and their inhibitors (Cdkn) found amongst upregulated genes and downregulated genes in different populations of G3+ cells.

\section{5-6- Gata3 expression is associated with a more quiescent cell state:}

Cell cycle refers to the process by which a cell duplicates its DNA in order to produce two daughter cells. The cell cycle is divided into 4 stages: G1, S, G2, and M. The G1 stage stands for the Gap 1 stage, where cells can either go to G0 and are not consider in cell cycle anymore, or go into stage S. Stage S refers to synthesis of the DNA, or the DNA replication which is followed by stage G2 or Gap 2. This is stage is then followed by M stage, which stands for mitosis where nuclear and cytoplasmic division happens, forming the two daughter cells. These cells then can either go into phase G1 again to start a new cycle or exit the cell cycle.

HSCs function and phenotype varies according to the time and tissue during ontogeny; In the AGM, the pre-HSCs are associated more with a proliferative status. However, once these cells start to acquire definitive HSCs state, they slow down cycling (Batsivari: 2017). In fetal liver, the HSCs are highly 
proliferative and are in cycle (Morrison: 1995), whereas the HSCs in the BM, cycle infrequently and are mostly quiescent (Wilson: 2008).

Considering that, we performed cell cycle analysis on the sorted populations obtained from G3-GFP embryos, E10.5 for HCs and ECs, and E11.5 for SNS cells and MCs, and found that Gata3 expression affected each population differently.

A
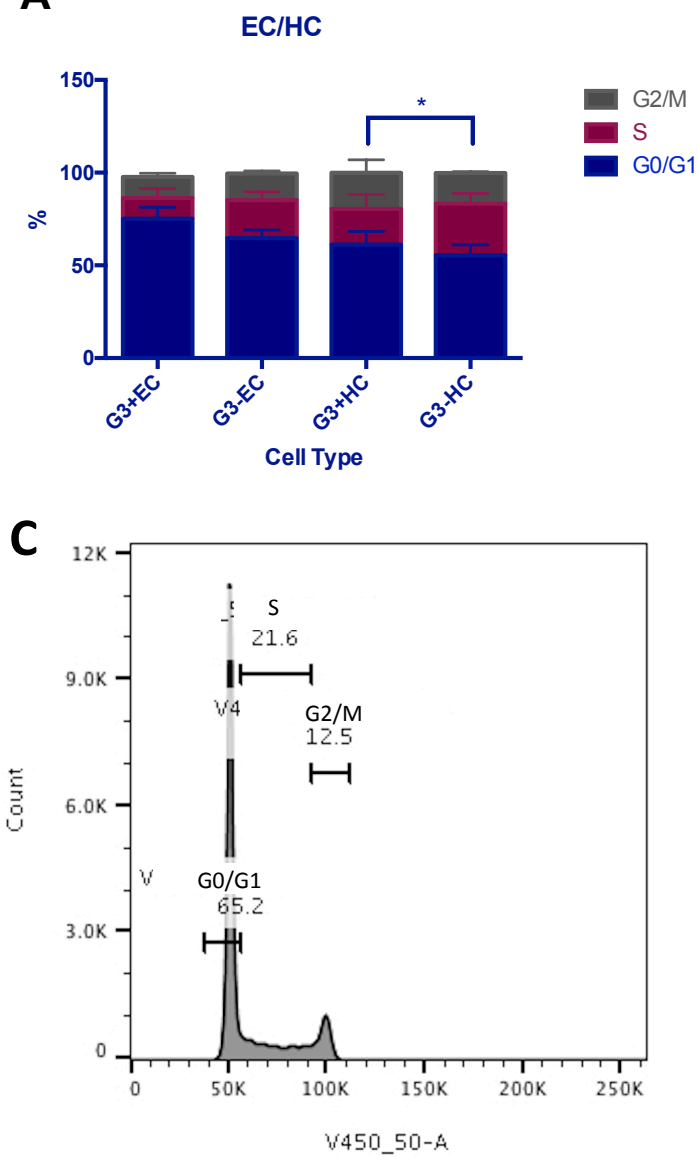

B

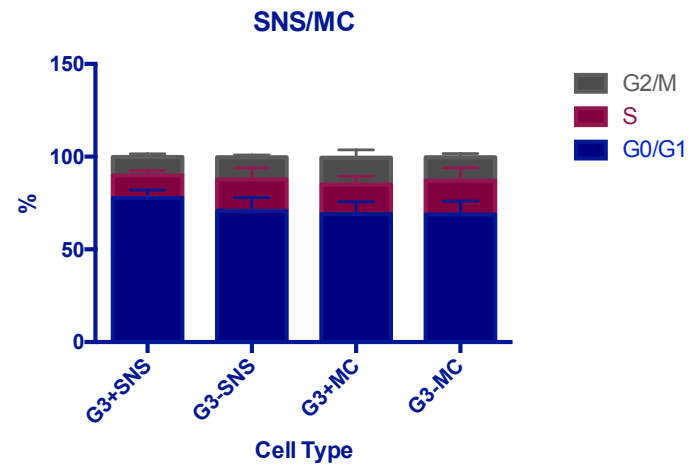

Figure 5-7: Cell cycle phase distribution of Gata3+ and Gata3-cells in the different cell populations in the AGM, where: (A) G3+EC: Gata3+ endothelial cells, G3-EC: Gata3- endothelial cells, G3+HC: Gata3+ haematopoietic cells, G3-HC: Gata3haematopoietic cells, (B) G3+SNS: Gata3+ sympathetic nervous system, G3-SNS: Gata3- sympathetic nervous system, G3+MC: Gata3+ mesenchymal cells, G3-MC: Gata3- mesenchymal cells C: . $n=3-4$ independent experiments, Statistical analysis were done using Mann-Whitney test. 
In the EC compartment, Gata3 expression was found to be associated mainly with cells in the G0/G1 phase, around 75\%, while 11\% of Gata3+ ECs were in the S phase, and 11\% were in the G2/M phase. G3- ECs had fewer cells in the G0/G1 phase, around $64 \%$, with $20.5 \%$ of the cells in the S phase and $14.4 \%$ in the G2/M phase (Figure 5-7 A).

Within the HCs, Gata3 expression was associated with $61 \%$ of the cells in the G0/G1 phase, around $20 \%$ in the $\mathrm{S}$ phase and $19.4 \%$ in the $\mathrm{G} 2 / \mathrm{M}$ phase. In G3-HCs, on the other hand, 55\% were found in the G0/G1 phase (which was significantly lower than the G0/G1 phase in $\mathrm{G} 3+\mathrm{HC}$ ), $27.7 \%$ in the S phase and $16.5 \%$ were in the G2/M phase (Figure 5-7A).

In SNS cells 77.7\% Gata3+ cells were found in the G0/G1 phase, $12.2 \%$ in the S phase, and $10 \%$ in the G2/M phase, while, in contrast, $70 \%$ of the G3-SNS was in the G0/G1 phase, $17 \%$ were in the S phase, and $12 \%$ were in the $\mathrm{G} 2 / \mathrm{M}$ phase (Figure 5-7 B). Finally, the cell cycle distribution of Gata3+ and Gata3- MCs was more or less equal. $68.9 \%$ of G3+MCs were found to reside in the G0/G1 phase, $15.9 \%$ in the $S$ phase, and $14.6 \%$ in the G2/M phase, which is similar to Gata3- cells, where $68.8 \%$ are in the G0/G1 phase, $18.2 \%$ in the S phase and $12.6 \%$ in the $\mathrm{G} 2 / \mathrm{M}$ phase.

However, it should be noted here that while the cells in the Gata3compartment were more abundant and formed a clear consistent profile in the cell cycle analysis, as it requires over 10,000 cells, Gata3-expressing populations were much more scarce; around 1000 cells for G3+ECs and less than 400 cells for G3+HC.

\section{5-7- Discussion:}

In this chapter, we performed RNA-Seq analysis to gain more insight into what distinguishes Gata3-expressing cells from non-expressing cells within the EC, HC, MC and SNS compartments. When we looked at the PCA, each of our 8 populations formed a clear cluster. As one would expect, the clustering was more 
prominent when we looked at each population individually: HC, EC, SNS, and MC, where Gata3-expressing cells formed a subpopulation distinct from the G3populations.

Most of the GO terms associated with the genes that were found to be enriched in these populations were related to general developmental processes, migration, and differentiation of various cells and systems. This is to be expected given the nature of the developing embryo and highlights the fact that most of these cells will go through various changes whether it is epithelial to mesenchymal transition, endothelial to haematopoietic transition, or the migration of neural crest cells to form the SNS.

For example, the cell migration and motility genes enriched in ECs could participate in the EHT that the cells must undergo before forming haematopoietic cells, which involves major morphological changes. Cells start to bud from the endothelial cells lining the walls of the dorsal aorta, forming intraaortic clusters before being released as HCs.

Also enriched in the GO terms associated with Gata3-expressing endothelial cells were the regulation of the transforming growth factor $\mathrm{b}$ (TGF-b) pathway and Wnt signalling. Both have been implicated before as regulators of haematopoietic cell emergence (Challan: 2010; Nostro: 2008).

Of note, the upregulated genes expressed in the G3+ ECs were most significantly enriched with genes that are associated with blood vessel formation and angiogenesis. These findings were similar to the findings in (Solaimani Kartalaei: 2015), where they reported that angiogenesis-related receptors, as Plxnd1, Eltd1, Calcrl, Ramp2, and S1pr1, play a role in activation of the haematopoietic potential in endothelial cells and the generation of HE.

Furthermore, among the upregulated genes in the G3+EC were genes that were associated with the early specification of the haematopoietic system as Gata2, which is a master regulator of haematopoiesis (Ling: 2004), Sox17 which is an intrinsic regulators of haemogenic endothelium specification and EHT (Clarke: 2013), BMP which is essential for the haemogenic endothelium cell 
formation and HSCs initiation (Zhang: 2014), and Meis1 which regulates the haemogenic endothelium generation (Wang: 2018). Taken together, this confirming that G3+ ECs are part of the HE.

Amongst the differentially expressed genes, we found members of the Cdk family and the Cdkn family expressed in HC, SNS and EC. We therefore decided to investigate if Gata3 plays a role in regulating the cell cycle of these cell types.

Except for MCs, where there was no major difference in the cell cycle state between the G3+ and G3- compartment, all of the other populations displayed a slight change in their proliferative status based on the Gata3 expression. In endothelial cells, the G3+ subpopulation was more highly represented in the non-proliferative stage (75\%) as compared with the G3-EC compartment (64\%).

Similarly, in the HC population, G3+ cells were mainly in the nonproliferative stage (61\%), which was slightly higher than the 55\% of the G3-HCs. Interestingly, it was reported recently by Batsivari et al. (2017), that the PreHSCs of E10.5 AGMs were mainly in the non-proliferative stage.

Similarly, in the SNS, the percentage of G3+ cells that were found in the quiescent stage was slightly higher than that of the G3-SNS, $77.7 \%$ and $70 \%$, respectively, which corresponds with what was reported by Chan et al. (2016), of most of the sympathoadrenal cells being found in the quiescent stage prior to the specification of those cells.

Given that there were no Cdks expressed in the MCs in both G3+ and G3compartments, we did not expect any major differences between the two populations. Therefore, as expected, the smallest difference between all populations was found in the MC. G3+MC had $68.9 \%$ in the non-proliferative stage whereas G3-MC had 68.8\%.

The fact that we found $75 \%$ of the G3+EC in the G0/G1 phase is interesting as previous studies have shown that the duration of the G1 phase is a determinant of stem cell fate (Hindley: 2013; Kueh: 2013; Pauklin: 2013). This agrees with what was proposed before by Gritz et al. (2016) in their review, that 
the endothelial cells must be arrested in G1 phase in order to undergo haemogenic specification (Gritz: 2016). 


\section{6- Discussion and future directions}

Haematopoietic stem and progenitor cells emerge from a subset of endothelial cells, termed haemogenic endothelium, which is found lining the walls of the dorsal aorta from about E9.5 until E11.5. The immediate microenvironment that provides a niche for HSCs and regulates the endothelial to haematopoietic transition also harbours a network of signals coming from various tissues, where these are controlled by transcription factors. One such transcription factor that is expressed in various tissues within the AGM is Gata3. Corresponding with what was reported before, we have confirmed that Gata3 is expressed in the SNS (Fitch: 2012), mesenchymal cells (Manaia: 2000), endothelial and haematopoietic cells (Swiers: 2013).

Here, we have further characterised the expression of Gata3 and found that it varied between different cells based on the embryonic age. At E11.5 Gata3 is expressed highly in the SNS and mesenchymal cells, to a lower extent in endothelial cells, and not expressed in haematopoietic cells. At E10.5, Gata3 is expressed in more endothelial cells, but to a lesser extent in SNS and mesenchymal cells. At this stage, Gata3 was also expressed in a transient haematopoietic population that disappeared at E11.5.

In order to investigate the haematopoietic potential of endothelial and haematopoietic populations, we used the OP9 co-culture system in conditions that support the formation of haematopoietic progenies. Within the endothelial compartment, we found that Gata3-expressing cells were enriched for haemogenic endothelium, and produced a higher number of haematopoietic progenitors when the cultured cells were plated into methylcellulose culture.

Within the haematopoietic compartments, we have found that Gata3expressing haematopoietic cells had a significant haematopoietic potential that paralleled that of the non-Gata3 expressing cells. In addition, when plated into methylcellulose, albeit the colonies that were produced were significantly fewer than those produced by G3-HC, the progenitors produced by G3+HC were 
enriched in CFU-Mix, derived from the most primitive and stem cell-like progenitor. In fact, with the exception of a few CFU-GM colonies, most of the progenitors produced by these cells were CFU-Mix.

Given the transient nature of G3+HC and their intriguing output in CFU-C after OP9 co-culture, we wanted to further investigate the origins and function of these cells. We performed CFU-C on freshly sorted uncultured cells obtained from E10.5 G3-GFP embryos. The only compartment that produced haematopoietic progenitors was that of the G3-HC. As expected, neither of the endothelial cell populations (G3+EC and G3-EC) produced any colonies. In contrast to both co-aggregates and co-culture, G3+HC did not produce any colonies suggesting that these progenitors are at such a rare frequency that they require a few days in culture in order to expand, mature, and give a read-out in CFU-C assays.

To further investigate these cells and their place within the developmental pathway towards the generation of dHSCs we used the coaggregate system; a system that was developed by the Medvinsky group to promote the development of pro/pre-HSCs to dHSCs. Haematopoietic cell output from this system is much higher than that produced by OP9 co-culture; however, the progenitors produced by Gata3+ cells had fewer CFU-Mix amongst them in contrast to the colonies produced after OP9 co-culture. Yet overall the colony count was similar to that obtained from G3-HC.

As one would suspect that neither G3+ nor G3- endothelial cells produced any colonies in methylcellulose after plating them directly from freshly sorted AGMs, and the haematopoietic colonies produced in methylcellulose after the coaggregates were few in contrast to those produced after OP9 co-culture. Considering the difference in the duration of OP9 co-culture (2-3 weeks) compared with the co-aggregates (4-5 days), this suggests that the endothelial to haematopoietic transition is still at its early stages and that only few cells were produced. 
To study HSC maturation, we transplanted cells obtained from E11.5 and E10.5 AGM co-aggregates. At both stages, there was no repopulation from either G3+EC or G3-EC. However, that was not the case for the haematopoietic cells. For haematopoietic cells harvested from E11.5 co-aggregates, only the G3-HC compartment repopulated the recipients. This was in contrast to E10.5, where only G3+HC could engraft irradiated mice. A number of conclusions can be drawn from this experiment: (A) that there are no Gata3-expressing HSCs in E11.5 embryos; (B) the presence of either pro/pre-HSCs within E10.5 G3+HCs that matured into HSCs after the co-aggregates, (3) the presence of dHSCs and/or pre-HSCs (I/II) in G3-HCs. However, given that the markers were pooled in the same channel (CD41/43/45) we could not specify at which stages those cells are. Therefore, more experiments are needed in the future to investigate the different subpopulations of Gata3+HC, and also identify the stages of these G3+HC and place them in the dHSCs developmental lineage.

These results implicated Gata3 in haematopoietic progenitor formation. Taken together with the previously reported effect of Gata3 germline deletion on HSCs (Fitch: 2012), we wanted to look at the effect of Gata3 global deletion on haematopoietic progenitor formation. Interestingly, we found that Gata3 deletion affects progenitor counts in a dose- and stage-dependent manner: i.e. heterozygous AGMs produced a lower count, which was significantly further reduced in knockout AGMs, and the effect of the deletion was more severe at E10.5. This dose-dependent effect of Gata3 was also reported in other systems; in thymocyte development (Ho: 2009), in kidney development (Grote: 2008), in SNS development (Pandolfi: 1995 ; Lim: 2000 ), in hair and skin development (Kaufman: 2003; de Guzman Strong: 2006; Kurek: 2007) and in mammary gland development (Kouros-Mehr: 2006; Asselin-Labat: 2007).

We then added an explant step in order to eliminate the variation between different biological replicates, thus giving a more accurate representation of progenitor numbers in the AGM. The effect of deletion was maintained, yet appeared more pronounced, since wild-type progenitors were able to expand during the explant culture step. The inability of both haploinsufficient and knockout progenitors to expand could be contributed to an 
involvement of Gata3 in cell cycle regulation, which corresponds with what was reported by $\mathrm{Ku}$ et al. of Gata3 knock-out HSCs failing to enter the cell cycle (Ku: 2007).

In order to investigate if Gata3 plays a more direct role in haemogenic endothelium and HSC and progenitor formation, we used the Vec-Cre system (Chen: 2009) to delete Gata3 from the vascular endothelium. The results we got from conditional deletion of Gata3 from endothelium were strikingly similar to those we got from germline deletion: Gata3 deletion affected progenitor numbers in a dose-dependent and stage-dependent manner; thus, showing that Gata3 within the haemogenic endothelium plays a major role in haematopoietic progenitor formation.

Given that both haematopoietic progenitors and HSCs emerge from haemogenic endothelium, we also investigated the effect of Gata3 conditional deletion from endothelial cells on haematopoietic repopulation via transplantation assay and found that indeed Gata3 plays a role in HSC generation. When Gata3 was deleted from the endothelium, there was almost no HSC engraftment in irradiated recipients. There was some repopulation in some of the mice, which could be attributed to a few HSCs that might have escaped Gata3 deletion in those AGMs, as we observed in some colonies in CFU-C assays. However, this retained activity in some of the HSCs in Gata3-null AGMs was also reported by Fitch et al. (2012), suggesting that while Gata3 plays a key role in HSC and progenitor formation, its role in EHT does not seem to be absolutely essential.

It is interesting that the effect of endothelial-specific Gata3 deletion on progenitor and HSC numbers is very similar to the effect observed with a global knockout, suggesting the function of Gata3 in the HE is more relevant to AGM haematopoiesis than its function in the SNS. It may also indicate that the role of Gata3 in the HE is temporally upstream of the role of Gata3 in the SNS, with the former regulating HSC formation and the latter HSC maintenance and/or function after these have been generated. The rescue effect we have previously observed with catecholamine addition (Fitch: 2012) may stem from an increased 
survival or expansion of those few HSCs that are detected in Vec-Cre deleted embryos.

To gain further insight into the role of Gata3 in the different AGM cell populations, we preformed a whole transcriptome analysis for small numbers of each of the Gata3-expressing subpopulations (EC, HC, MC and SNS), and compared them to their Gata3-negative counterparts. RNA-Seq analysis revealed that Gata3+ and Gata3- cells segregated into distinct clusters in the PCA analysis, thus revealing that Gata3-expressing cells share a common genetic profile that differentiates them from Gata3-non expressing cells.

The genes that differentiate between the Gata3+ and Gata3- fractions within each cell population were classified according to the biological processes they are involved in using Gene Ontology analysis. All Gata3-expressing populations shared a common theme in the gene enrichment analysis in both upregulated and downregulated genes. Mainly, the downregulated genes were associated with cell development, differentiation and specification of various cells and systems, whereas the upregulated genes were mostly associated with migration, proliferation, development and apoptosis. Of note, genes that were associated with blood vessel formation and angiogenesis such as Hey1, Efnb2, and Nrp1 (Zhou:2016; Solaimani Kartalaei: 2015) were found in G3+EC in the upregulated genes. These genes have been reported before to be enriched in the haemogenic endothelium and are associated with EHT. Other genes upregulated in G3+ECs that have previously been reported to be enriched in HEC included Mecom, Notch4, Notch1, Mapk3, Sox17, Epas1, Hey2, Sox7, Sox17, Nkx2-3, Tsc22d1, Nfic, and Pdlim1 (Solaimani Kartalaei: 2015). This implicates Gata3 as a key regulator for HE and EHT. However, given that G3-EC are still capable of producing haematopoietic progeny, it would be interesting to see if Gata3 marks a subtype of $\mathrm{HE}$, and if that subfraction is responsible for a certain type of haematopoietic cells or may represent HECs at different stages of the cell cycle.

In addition, we found that all Gata3-expressing cell populations, except mesenchymal cells, had amongst their differentially expressed genes cyclindependent kinases or their inhibitor, known regulators of the cell cycle. Given 
what was reported before of Gata3 involvement in cell cycle regulation (Ku: 2007), we wanted to further explore a possible link of Gata3 with the cell cycle within the different AGM populations. We found that Gata3 expression is associated with more quiescent cells in the AGM, which goes with the fact that almost all of the upregulated cell cycle genes were cyclin-dependent kinase inhibitors, whereas cyclin dependent-kinases were amongst the downregulated genes.

The cell cycle status of AGM HSCs and their precursors has recently been analysed by the Medvinsky group. They found that pre-HSCs could be either quiescent or proliferating, depending on the developmental time point and whether it was pre-HSCs I or II (Batsivari: 2017). It will be interesting to determine whether Gata3 is one of the factors controlling the cycling status of these cells.

Within the haemogenic endothelium, we found that Gata3 is also associated with more quiescent cells. The specification of haemogenic endothelium was suggested to happen during an arrest in the G1 phase (Gritz: 2016), and some studies have shown that the length of the G1 phase is a determinant of stem cell fate (Hindley: 2013; Kueh: 2013; Pauklin: 2013). In addition, gene transcriptome analysis has also revealed that several haematopoietic transcriptional regulators were expressed in the G1 phase (Aggarwal: 2012). Along with our functional studies, this further implicates Gata3 as an EHT regulator.

Within the sympathoadrenal cells, similar to both HC and EC, Gata3expressing cells were associated with the quiescent phase, though the effect was more pronounced in this population, which corresponds with the findings of Chan et al. (2016) of sympathoadrenal cells exiting the cell cycle at E11.5 prior to their specification and differentiation.

In mesenchymal cells, there was no significant difference in cell cycle status between Gata3-expressing cells and Gata3 negative cells, which was expected given that there was no differential expression of cyclin-dependent 
kinases or their inhibitors. However, given the location of these cells in the subaortic mesenchyme, i.e. in close proximity to where HSCs emerge, it would still be interesting to further investigate the role of these cells in HSC and progenitor generation, possibly via the Gata3-dependent secretion of HSC supportive factors.

The role of Gata3 as a cell cycle regulator could potentially explain its role as a tumour suppressor gene in several types of cancer. In breast cancer, certain types of renal carcinoma, and certain types of leukaemia, Gata3 was found to stop cancer progression and dissemination, and its absence was associated with poor prognosis and more aggressive disease (Kouros-Mehr: 2008; Cooper: 2008; Zhang: 2012).

In addition, given our findings of Gata3 association with more quiescent cells, and the presence of upregulated cyclin dependent kinase inhibitors in Gata3-expressing cells, one would expect Gata3 deletion to result in acute cell proliferation, in contrary to our findings in CFU-C assays, where the absence of Gata3 led to a severe reduction in colony counts. However, considering what was reported before by Frelin et al (2013), that Gata3 has to translocate from the cytoplasm to the nucleus to be active and functional in the cell, my results remain within context. Taken together, this indicates a very complex relationship between Gata3, cell differentiation and the cell cycle.

In summary, Gata3 is essential for the development of several types of cells, organs and tissues, and its disruption during development results in severe defects and impairment in those systems, leading to embryonic death at midgestation following germline deletion. In the haematopoietic system, we have found that Gata3-expressing cells are enriched for haemogenic endothelium and play a critical role in the formation of haematopoietic stem and progenitors cells. In addition, we observed that Gata3 marks specific stages along the developmental pathway towards the generation of dHSCs. Within the HSC microenvironment, Gata3 plays a major role in the development of various cells and systems, which, as in the case of the SNS, can indirectly impact on the 
haematopoietic system. Many of these effects may be linked to the involvement of Gata3 in cell cycle regulation. 


\section{References}

Adamo L, Naveiras O, Wenzel PL, McKinney-Freeman S, Mack PJ, GraciaSancho J, Suchy-Dicey A, Yoshimoto M, Lensch MW, Yoder MC and others. 2009. Biomechanical forces promote embryonic haematopoiesis. Nature 459(7250):1131-1135.

Aggarwal R, Lu J, Pompili VJ, Das H. 2012. Hematopoietic stem cells: transcriptional regulation, ex vivo expansion and clinical application. Curr Mol Med 12(1):34-49.

Ali AK, Oh JS, Vivier E, Busslinger M, Lee SH. 2016. NK Cell-Specific Gata3 Ablation Identifies the Maturation Program Required for Bone Marrow Exit and Control of Proliferation. J Immunol 196(4):1753-1767.

Asselin-Labat ML, Sutherland KD, Barker H, Thomas R, Shackleton M, Forrest NC, Hartley L, Robb L, Grosveld FG, van der Wees J and others. 2007. Gata-3 is an essential regulator of mammary-gland morphogenesis and luminalcell differentiation. Nat Cell Biol 9(2):201-209.

Azzoni E, Conti V, Campana L, Dellavalle A, Adams RH, Cossu G, Brunelli S. 2014. Hemogenic endothelium generates mesoangioblasts that contribute to several mesodermal lineages in vivo. Development 141(9):1821-1834.

Bates DL, Chen Y, Kim G, Guo L, Chen L. 2008. Crystal structures of multiple GATA zinc fingers bound to DNA reveal new insights into DNA recognition and self-association by GATA. J Mol Biol 381(5):1292-1306. 
Batsivari A, Rybtsov S, Souilhol C, Binagui-Casas A, Hills D, Zhao S, Travers P, Medvinsky A. 2017. Understanding Hematopoietic Stem Cell Development through Functional Correlation of Their Proliferative Status with the Intra-aortic Cluster Architecture. Stem Cell Reports 8(6):1549-1562.

Benveniste P, Frelin C, Janmohamed S, Barbara M, Herrington R, Hyam D, Iscove NN. 2010. Intermediate-term hematopoietic stem cells with extended but time-limited reconstitution potential. Cell Stem Cell 6(1):48-58.

Bertrand JY, Chi NC, Santoso B, Teng S, Stainier DY, Traver D. 2010. Haematopoietic stem cells derive directly from aortic endothelium during development. Nature 464(7285):108-111.

Bertrand JY, Giroux S, Golub R, Klaine M, Jalil A, Boucontet L, Godin I, Cumano A. 2005. Characterization of purified intraembryonic hematopoietic stem cells as a tool to define their site of origin. Proc Natl Acad Sci U S A 102(1):134-139.

Bertwistle D, Walmsley ME, Read EM, Pizzey JA, Patient RK. 1996. GATA factors and the origins of adult and embryonic blood in Xenopus: responses to retinoic acid. Mech Dev 57(2):199-214.

Boiers C, Carrelha J, Lutteropp M, Luc S, Green JC, Azzoni E, Woll PS, Mead AJ, Hultquist A, Swiers G and others. 2013. Lymphomyeloid contribution of an immune-restricted progenitor emerging prior to definitive hematopoietic stem cells. Cell Stem Cell 13(5):535-548.

Boisset JC, Robin C. 2012. On the origin of hematopoietic stem cells: progress and controversy. Stem Cell Res 8(1):1-13.

Boisset JC, van Cappellen W, Andrieu-Soler C, Galjart N, Dzierzak E, Robin C. 2010. In vivo imaging of haematopoietic cells emerging from the mouse aortic endothelium. Nature 464(7285):116-120. 
Bresnick EH, Lee HY, Fujiwara T, Johnson KD, Keles S. 2010. GATA switches as developmental drivers. J Biol Chem 285(41):31087-31093.

Burch JB. 2005. Regulation of GATA gene expression during vertebrate development. Semin Cell Dev Biol 16(1):71-81.

Burda P, Vargova J, Curik N, Salek C, Papadopoulos GL, Strouboulis J, Stopka T. 2016. GATA-1 Inhibits PU.1 Gene via DNA and Histone H3K9 Methylation of Its Distal Enhancer in Erythroleukemia. PLoS One 11(3):e0152234.

Butko E, Pouget C, Traver D. 2016. Complex regulation of HSC emergence by the Notch signaling pathway. Dev Biol 409(1):129-138.

Buza-Vidas N, Duarte S, Luc S, Bouriez-Jones T, Woll PS, Jacobsen SE. 2011. GATA3 is redundant for maintenance and self-renewal of hematopoietic stem cells. Blood 118(5):1291-1293.

Cai Z, de Bruijn M, Ma X, Dortland B, Luteijn T, Downing RJ, Dzierzak E. 2000. Haploinsufficiency of AML1 affects the temporal and spatial generation of hematopoietic stem cells in the mouse embryo. Immunity 13(4):423-431.

Cancer Genome Atlas N. 2012. Comprehensive molecular portraits of human breast tumours. Nature 490(7418):61-70.

Cerdan C, Bhatia M. 2010. Novel roles for Notch, Wnt and Hedgehog in hematopoesis derived from human pluripotent stem cells. Int J Dev Biol 54(67):955-963.

Challen GA, Boles NC, Chambers SM, Goodell MA. 2010. Distinct hematopoietic stem cell subtypes are differentially regulated by TGF-beta1. Cell Stem Cell 6(3):265-278. 
Chan CK, Lindau P, Jiang W, Chen JY, Zhang LF, Chen CC, Seita J, Sahoo D, Kim JB, Lee A and others. 2013. Clonal precursor of bone, cartilage, and hematopoietic niche stromal cells. Proc Natl Acad Sci U S A 110(31):1264312648.

Chan WH, Gonsalvez DG, Young HM, Southard-Smith EM, Cane KN, Anderson CR. 2016. Differences in CART expression and cell cycle behavior discriminate sympathetic neuroblast from chromaffin cell lineages in mouse sympathoadrenal cells. Dev Neurobiol 76(2):137-149.

Chang AN, Cantor AB, Fujiwara Y, Lodish MB, Droho S, Crispino JD, Orkin SH. 2002. GATA-factor dependence of the multitype zinc-finger protein FOG-1 for its essential role in megakaryopoiesis. Proc Natl Acad Sci U S A 99(14):92379242.

Chen MJ, Li Y, De Obaldia ME, Yang Q, Yzaguirre AD, Yamada-Inagawa T, Vink CS, Bhandoola A, Dzierzak E, Speck NA. 2011. Erythroid/myeloid progenitors and hematopoietic stem cells originate from distinct populations of endothelial cells. Cell Stem Cell 9(6):541-552.

Chen MJ, Yokomizo T, Zeigler BM, Dzierzak E, Speck NA. 2009. Runx1 is required for the endothelial to haematopoietic cell transition but not thereafter. Nature 457(7231):887-891.

Chen T, Wang F, Wu M, Wang ZZ. 2015. Development of hematopoietic stem and progenitor cells from human pluripotent stem cells. J Cell Biochem 116(7):1179-1189.

Cheng T, Rodrigues N, Shen H, Yang Y, Dombkowski D, Sykes M, Scadden DT. 2000. Hematopoietic stem cell quiescence maintained by p21cip1/waf1. Science 287(5459):1804-1808. 
Chiba T, Nagata Y, Kishi A, Sakamaki K, Miyajima A, Yamamoto M, Engel JD, Todokoro K. 1993. Induction of erythroid-specific gene expression in lymphoid cells. Proc Natl Acad Sci U S A 90(24):11593-11597.

Chotinantakul K, Leeanansaksiri W. 2012. Hematopoietic stem cell development, niches, and signaling pathways. Bone Marrow Res 2012:270425.

Chou J, Lin JH, Brenot A, Kim JW, Provot S, Werb Z. 2013. GATA3 suppresses metastasis and modulates the tumour microenvironment by regulating microRNA-29b expression. Nat Cell Biol 15(2):201-213.

Chou J, Provot S, Werb Z. 2010. GATA3 in development and cancer differentiation: cells GATA have it! J Cell Physiol 222(1):42-49.

Chow A, Lucas D, Hidalgo A, Mendez-Ferrer S, Hashimoto D, Scheiermann C, Battista M, Leboeuf M, Prophete C, van Rooijen N and others. 2011. Bone marrow CD169+ macrophages promote the retention of hematopoietic stem and progenitor cells in the mesenchymal stem cell niche. J Exp Med 208(2):261-271.

Ciau-Uitz A, Monteiro R, Kirmizitas A, Patient R. 2014. Developmental hematopoiesis: ontogeny, genetic programming and conservation. Exp Hematol 42(8):669-683.

Clarke RL, Yzaguirre AD, Yashiro-Ohtani Y, Bondue A, Blanpain C, Pear WS, Speck NA, Keller G. 2013. The expression of Sox17 identifies and regulates haemogenic endothelium. Nat Cell Biol 15(5):502-510.

Cooper SJ, Zou H, Legrand SN, Marlow LA, von Roemeling CA, Radisky DC, Wu KJ, Hempel N, Margulis V, Tun HW and others. 2010. Loss of type III transforming growth factor-beta receptor expression is due to methylation silencing of the transcription factor GATA3 in renal cell carcinoma. Oncogene 29(20):2905-2915. 
Corbel C, Salaun J. 2002. AlphaIIb integrin expression during development of the murine hemopoietic system. Dev Biol 243(2):301-311.

Dallaire A, Simard MJ. 2016. The implication of microRNAs and endosiRNAs in animal germline and early development. Dev Biol 416(1):18-25.

de Bruijn MF, Ma X, Robin C, Ottersbach K, Sanchez MJ, Dzierzak E. 2002. Hematopoietic stem cells localize to the endothelial cell layer in the midgestation mouse aorta. Immunity 16(5):673-683.

de Bruijn MF, Speck NA, Peeters MC, Dzierzak E. 2000. Definitive hematopoietic stem cells first develop within the major arterial regions of the mouse embryo. EMBO J 19(11):2465-2474.

de Graaf CA, Kauppi M, Baldwin T, Hyland CD, Metcalf D, Willson TA, Carpinelli MR, Smyth GK, Alexander WS, Hilton DJ. 2010. Regulation of hematopoietic stem cells by their mature progeny. Proc Natl Acad Sci U S A 107(50):21689-21694.

de Guzman Strong C, Wertz PW, Wang C, Yang F, Meltzer PS, Andl T, Millar SE, Ho IC, Pai SY, Segre JA. 2006. Lipid defect underlies selective skin barrier impairment of an epidermal-specific deletion of Gata-3. J Cell Biol 175(4):661670.

De la Chapelle A, Fantoni A, Marks PA. 1969. Differentiation of mammalian somatic cells: DNA and hemoglobin synthesis in fetal mouse yolk sac erythroid cells. Proc Natl Acad Sci U S A 63(3):812-819.

Deichmann U. 2016. Epigenetics: The origins and evolution of a fashionable topic. Dev Biol 416(1):249-254.

Dennis JE, Charbord P. 2002. Origin and differentiation of human and murine stroma. Stem Cells 20(3):205-214. 
Dianati E, Poiraud J, Weber-Ouellette A, Plante I. 2016. Connexins, Ecadherin, Claudin-7 and beta-catenin transiently form junctional nexuses during the post-natal mammary gland development. Dev Biol 416(1):52-68.

Ding L, Saunders TL, Enikolopov G, Morrison SJ. 2012. Endothelial and perivascular cells maintain haematopoietic stem cells. Nature 481(7382):457462.

Durand C, Dzierzak E. 2005. Embryonic beginnings of adult hematopoietic stem cells. Haematologica 90(1):100-108.

Durand C, Robin C, Bollerot K, Baron MH, Ottersbach K, Dzierzak E. 2007. Embryonic stromal clones reveal developmental regulators of definitive hematopoietic stem cells. Proc Natl Acad Sci U S A 104(52):20838-20843.

Dydensborg AB, Rose AA, Wilson BJ, Grote D, Paquet M, Giguere V, Siegel PM, Bouchard M. 2009. GATA3 inhibits breast cancer growth and pulmonary breast cancer metastasis. Oncogene 28(29):2634-2642.

Dykstra B, Kent D, Bowie M, McCaffrey L, Hamilton M, Lyons K, Lee SJ, Brinkman R, Eaves C. 2007. Long-term propagation of distinct hematopoietic differentiation programs in vivo. Cell Stem Cell 1(2):218-229.

Dzierzak E, Speck NA. 2008. Of lineage and legacy: the development of mammalian hematopoietic stem cells. Nat Immunol 9(2):129-136.

Eeckhoute J, Keeton EK, Lupien M, Krum SA, Carroll JS, Brown M. 2007. Positive cross-regulatory loop ties GATA-3 to estrogen receptor alpha expression in breast cancer. Cancer Res 67(13):6477-6483.

Eilken HM, Nishikawa S, Schroeder T. 2009. Continuous single-cell imaging of blood generation from haemogenic endothelium. Nature 457(7231):896-900. 
Endoh M, Ogawa M, Orkin S, Nishikawa S. 2002. SCL/tal-1-dependent process determines a competence to select the definitive hematopoietic lineage prior to endothelial differentiation. EMBO J 21(24):6700-6708.

Espin-Palazon R, Stachura DL, Campbell CA, Garcia-Moreno D, Del Cid N, Kim AD, Candel S, Meseguer J, Mulero V, Traver D. 2014. Proinflammatory signaling regulates hematopoietic stem cell emergence. Cell 159(5):1070-1085.

Faunes F, Larrain J. 2016. Conservation in the involvement of heterochronic genes and hormones during developmental transitions. Dev Biol 416(1):3-17.

Fitch SR, Kimber GM, Wilson NK, Parker A, Mirshekar-Syahkal B, Gottgens B, Medvinsky A, Dzierzak E, Ottersbach K. 2012. Signaling from the sympathetic nervous system regulates hematopoietic stem cell emergence during embryogenesis. Cell Stem Cell 11(4):554-566.

Foudi A, Hochedlinger K, Van Buren D, Schindler JW, Jaenisch R, Carey V, Hock H. 2009. Analysis of histone 2B-GFP retention reveals slowly cycling hematopoietic stem cells. Nat Biotechnol 27(1):84-90.

Frame JM, McGrath KE, Palis J. 2013. Erythro-myeloid progenitors: "definitive" hematopoiesis in the conceptus prior to the emergence of hematopoietic stem cells. Blood Cells Mol Dis 51(4):220-225.

Frelin C, Herrington R, Janmohamed S, Barbara M, Tran G, Paige CJ, Benveniste P, Zuniga-Pflucker JC, Souabni A, Busslinger M and others. 2013. GATA-3 regulates the self-renewal of long-term hematopoietic stem cells. Nat Immunol 14(10):1037-1044. 
Fujiwara T, O'Geen H, Keles S, Blahnik K, Linnemann AK, Kang YA, Choi K, Farnham PJ, Bresnick EH. 2009. Discovering hematopoietic mechanisms through genome-wide analysis of GATA factor chromatin occupancy. Mol Cell 36(4):667681.

Gao J, Chen YH, Peterson LC. 2015. GATA family transcriptional factors: emerging suspects in hematologic disorders. Exp Hematol Oncol 4:28.

Garcia-Alegria E, Menegatti S, Batta K, Cuvertino S, Florkowska M, Kouskoff V. 2016. Emerging concepts for the in vitro derivation of murine haematopoietic stem and progenitor cells. FEBS Lett 590(22):4116-4125.

Garcia-Porrero JA, Manaia A, Jimeno J, Lasky LL, Dieterlen-Lievre F, Godin IE. 1998. Antigenic profiles of endothelial and hemopoietic lineages in murine intraembryonic hemogenic sites. Dev Comp Immunol 22(3):303-319.

Gekas C, Dieterlen-Lievre F, Orkin SH, Mikkola HK. 2005. The placenta is a niche for hematopoietic stem cells. Dev Cell 8(3):365-375.

Gering M, Patient R. 2005. Hedgehog signaling is required for adult blood stem cell formation in zebrafish embryos. Dev Cell 8(3):389-400.

Golub R, Cumano A. 2013. Embryonic hematopoiesis. Blood Cells Mol Dis 51(4):226-231.

Gordon-Keylock S, Sobiesiak M, Rybtsov S, Moore K, Medvinsky A. 2013. Mouse extraembryonic arterial vessels harbor precursors capable of maturing into definitive HSCs. Blood 122(14):2338-2345.

Gregory T, Yu C, Ma A, Orkin SH, Blobel GA, Weiss MJ. 1999. GATA-1 and erythropoietin cooperate to promote erythroid cell survival by regulating bcl-xL expression. Blood 94(1):87-96. 
Grimbert S, Tietze K, Barkoulas M, Sternberg PW, Felix MA, Braendle C. 2016. Anchor cell signaling and vulval precursor cell positioning establish a reproducible spatial context during C. elegans vulval induction. Dev Biol 416(1):123-135.

Gritz E, Hirschi KK. 2016. Specification and function of hemogenic endothelium during embryogenesis. Cell Mol Life Sci 73(8):1547-1567.

Grote D, Boualia SK, Souabni A, Merkel C, Chi X, Costantini F, Carroll T, Bouchard M. 2008. Gata3 acts downstream of beta-catenin signaling to prevent ectopic metanephric kidney induction. PLoS Genet 4(12):e1000316.

Grote D, Souabni A, Busslinger M, Bouchard M. 2006. Pax 2/8-regulated Gata 3 expression is necessary for morphogenesis and guidance of the nephric duct in the developing kidney. Development 133(1):53-61.

Hadland BK, Varnum-Finney B, Mandal PK, Rossi DJ, Poulos MG, Butler JM, Rafii S, Yoder MC, Yoshimoto M, Bernstein ID. 2017. A Common Origin for B-1a and B-2 Lymphocytes in Clonal Pre- Hematopoietic Stem Cells. Stem Cell Reports 8(6):1563-1572.

Hadland BK, Varnum-Finney B, Poulos MG, Moon RT, Butler JM, Rafii S, Bernstein ID. 2015. Endothelium and NOTCH specify and amplify aorta-gonadmesonephros-derived hematopoietic stem cells. J Clin Invest 125(5):2032-2045.

Harigae H, Takahashi S, Suwabe N, Ohtsu H, Gu L, Yang Z, Tsai FY, Kitamura Y, Engel JD, Yamamoto M. 1998. Differential roles of GATA-1 and GATA2 in growth and differentiation of mast cells. Genes Cells 3(1):39-50.

Heavey B, Charalambous C, Cobaleda C, Busslinger M. 2003. Myeloid lineage switch of Pax5 mutant but not wild-type $B$ cell progenitors by C/EBPalpha and GATA factors. EMBO J 22(15):3887-3897. 
Hindley C, Philpott A. 2013. The cell cycle and pluripotency. Biochem J 451(2):135-143.

Ho IC, Pai SY. 2007. GATA-3 - not just for Th2 cells anymore. Cell Mol Immunol 4(1):15-29.

Ho IC, Tai TS, Pai SY. 2009. GATA3 and the T-cell lineage: essential functions before and after T-helper-2-cell differentiation. Nat Rev Immunol $9(2): 125-135$.

Hong SJ, Choi HJ, Hong S, Huh Y, Chae H, Kim KS. 2008. Transcription factor GATA-3 regulates the transcriptional activity of dopamine betahydroxylase by interacting with Sp1 and AP4. Neurochem Res 33(9):1821-1831.

Hosoya T, Maillard I, Engel JD. 2010. From the cradle to the grave: activities of GATA-3 throughout T-cell development and differentiation. Immunol Rev 238(1):110-125.

Huber P, Crum T, Okkema PG. 2016. Function of the C. elegans T-box factor TBX-2 depends on interaction with the UNC-37/Groucho corepressor. Dev Biol 416(1):266-276.

Iacovino M, Chong D, Szatmari I, Hartweck L, Rux D, Caprioli A, Cleaver O, Kyba M. 2011. HoxA3 is an apical regulator of haemogenic endothelium. Nat Cell Biol 13(1):72-78.

Imanirad P, Solaimani Kartalaei P, Crisan M, Vink C, Yamada-Inagawa T, de Pater E, Kurek D, Kaimakis P, van der Linden R, Speck N and others. 2014. HIF1alpha is a regulator of hematopoietic progenitor and stem cell development in hypoxic sites of the mouse embryo. Stem Cell Res 12(1):24-35.

Ivanovs A, Rybtsov S, Anderson RA, Turner ML, Medvinsky A. 2014. Identification of the niche and phenotype of the first human hematopoietic stem 
cells. Stem Cell Reports 2(4):449-456.

Iwasaki H, Mizuno S, Wells RA, Cantor AB, Watanabe S, Akashi K. 2003. GATA-1 converts lymphoid and myelomonocytic progenitors into the megakaryocyte/erythrocyte lineages. Immunity 19(3):451-462.

Jaffredo T, Bollerot K, Sugiyama D, Gautier R, Drevon C. 2005. Tracing the hemangioblast during embryogenesis: developmental relationships between endothelial and hematopoietic cells. Int J Dev Biol 49(2-3):269-277.

Jaffredo T, Gautier R, Eichmann A, Dieterlen-Lievre F. 1998. Intraaortic hemopoietic cells are derived from endothelial cells during ontogeny. Development 125(22):4575-4583.

Jaffredo T, Lempereur A, Richard C, Bollerot K, Gautier R, Canto PY, Drevon C, Souyri M, Durand C. 2013. Dorso-ventral contributions in the formation of the embryonic aorta and the control of aortic hematopoiesis. Blood Cells Mol Dis 51(4):232-238.

Jaffredo T, Nottingham W, Liddiard K, Bollerot K, Pouget C, de Bruijn M. 2005. From hemangioblast to hematopoietic stem cell: an endothelial connection? Exp Hematol 33(9):1029-1040.

Jang IH, Lu YF, Zhao L, Wenzel PL, Kume T, Datta SM, Arora N, Guiu J, Lagha M, Kim PG and others. 2015. Notch1 acts via Foxc2 to promote definitive hematopoiesis via effects on hemogenic endothelium. Blood 125(9):1418-1426.

Jones DL, Wagers AJ. 2008. No place like home: anatomy and function of the stem cell niche. Nat Rev Mol Cell Biol 9(1):11-21.

Jones JM, Warchol ME. 2009. Expression of the Gata3 transcription factor in the acoustic ganglion of the developing avian inner ear. J Comp Neurol 516(6):507-518. 
Joosten M, Seitz V, Zimmermann K, Sommerfeld A, Berg E, Lenze D, Leser U, Stein H, Hummel M. 2013. Histone acetylation and DNA demethylation of T cells result in an anaplastic large cell lymphoma-like phenotype. Haematologica 98(2):247-254.

Katayama Y, Battista M, Kao WM, Hidalgo A, Peired AJ, Thomas SA, Frenette PS. 2006. Signals from the sympathetic nervous system regulate hematopoietic stem cell egress from bone marrow. Cell 124(2):407-421.

Kaufman CK, Zhou P, Pasolli HA, Rendl M, Bolotin D, Lim KC, Dai X, Alegre ML, Fuchs E. 2003. GATA-3: an unexpected regulator of cell lineage determination in skin. Genes Dev 17(17):2108-2122.

Kent DG, Copley MR, Benz C, Wohrer S, Dykstra BJ, Ma E, Cheyne J, Zhao Y, Bowie MB, Zhao Y and others. 2009. Prospective isolation and molecular characterization of hematopoietic stem cells with durable self-renewal potential. Blood 113(25):6342-6350.

Kiel MJ, Radice GL, Morrison SJ. 2007. Lack of evidence that hematopoietic stem cells depend on $\mathrm{N}$-cadherin-mediated adhesion to osteoblasts for their maintenance. Cell Stem Cell 1(2):204-217.

Kiel MJ, Yilmaz OH, Iwashita T, Yilmaz OH, Terhorst C, Morrison SJ. 2005. SLAM family receptors distinguish hematopoietic stem and progenitor cells and reveal endothelial niches for stem cells. Cell 121(7):1109-1121.

Kim I, Saunders TL, Morrison SJ. 2007. Sox17 dependence distinguishes the transcriptional regulation of fetal from adult hematopoietic stem cells. Cell 130(3):470-483.

Kim I, Yilmaz OH, Morrison SJ. 2005. CD144 (VE-cadherin) is transiently expressed by fetal liver hematopoietic stem cells. Blood 106(3):903-905. 
Kina T, Ikuta K, Takayama E, Wada K, Majumdar AS, Weissman IL, Katsura Y. 2000. The monoclonal antibody TER-119 recognizes a molecule associated with glycophorin A and specifically marks the late stages of murine erythroid lineage. Br J Haematol 109(2):280-287.

Kingsley PD, Malik J, Emerson RL, Bushnell TP, McGrath KE, Bloedorn LA, Bulger M, Palis J. 2006. "Maturational" globin switching in primary primitive erythroid cells. Blood 107(4):1665-1672.

Kingsley PD, Malik J, Fantauzzo KA, Palis J. 2004. Yolk sac-derived primitive erythroblasts enucleate during mammalian embryogenesis. Blood 104(1):19-25.

Kissa K, Herbomel P. 2010. Blood stem cells emerge from aortic endothelium by a novel type of cell transition. Nature 464(7285):112-115.

Kobayashi H, Butler JM, O'Donnell R, Kobayashi M, Ding BS, Bonner B, Chiu VK, Nolan DJ, Shido K, Benjamin L and others. 2010. Angiocrine factors from Akt-activated endothelial cells balance self-renewal and differentiation of haematopoietic stem cells. Nat Cell Biol 12(11):1046-1056.

Kodama H, Nose M, Niida S, Nishikawa S, Nishikawa S. 1994. Involvement of the c-kit receptor in the adhesion of hematopoietic stem cells to stromal cells. Exp Hematol 22(10):979-984.

Kouros-Mehr H, Bechis SK, Slorach EM, Littlepage LE, Egeblad M, Ewald AJ, Pai SY, Ho IC, Werb Z. 2008. GATA-3 links tumor differentiation and dissemination in a luminal breast cancer model. Cancer Cell 13(2):141-152.

Kouros-Mehr H, Werb Z. 2006. Candidate regulators of mammary branching morphogenesis identified by genome-wide transcript analysis. Dev Dyn 235(12):3404-3412. 
Kouros-Mehr H, Werb Z. 2006. Candidate regulators of mammary branching morphogenesis identified by genome-wide transcript analysis. Dev Dyn 235(12):3404-3412.

Ku CJ, Hosoya T, Maillard I, Engel JD. 2012. GATA-3 regulates hematopoietic stem cell maintenance and cell-cycle entry. Blood 119(10):22422251.

Kueh HY, Champhekar A, Nutt SL, Elowitz MB, Rothenberg EV. 2013. Positive feedback between PU.1 and the cell cycle controls myeloid differentiation. Science 341(6146):670-673.

Kumaravelu P, Hook L, Morrison AM, Ure J, Zhao S, Zuyev S, Ansell J, Medvinsky A. 2002. Quantitative developmental anatomy of definitive haematopoietic stem cells/long-term repopulating units (HSC/RUs): role of the aorta-gonad-mesonephros (AGM) region and the yolk sac in colonisation of the mouse embryonic liver. Development 129(21):4891-4899.

Kurek D, Garinis GA, van Doorninck JH, van der Wees J, Grosveld FG. 2007. Transcriptome and phenotypic analysis reveals Gata3-dependent signalling pathways in murine hair follicles. Development 134(2):261-272.

Labastie MC, Cortes F, Romeo PH, Dulac C, Peault B. 1998. Molecular identity of hematopoietic precursor cells emerging in the human embryo. Blood 92(10):3624-3635.

Lacaud G, Kouskoff V. 2016. Hemangioblast, hemogenic endothelium, and primitive versus definitive hematopoiesis. Exp Hematol.

Lam EY, Hall CJ, Crosier PS, Crosier KE, Flores MV. 2010. Live imaging of Runx1 expression in the dorsal aorta tracks the emergence of blood progenitors from endothelial cells. Blood 116(6):909-914. 
Lancrin C, Mazan M, Stefanska M, Patel R, Lichtinger M, Costa G, Vargel O, Wilson NK, Moroy T, Bonifer C and others. 2012. GFI1 and GFI1B control the loss of endothelial identity of hemogenic endothelium during hematopoietic commitment. Blood 120(2):314-322.

Lancrin C, Sroczynska P, Stephenson C, Allen T, Kouskoff V, Lacaud G. 2009. The haemangioblast generates haematopoietic cells through a haemogenic endothelium stage. Nature 457(7231):892-895.

Ledbetter JA, Herzenberg LA. 1979. Xenogeneic monoclonal antibodies to mouse lymphoid differentiation antigens. Immunol Rev 47:63-90.

Lee GR, Fields PE, Flavell RA. 2001. Regulation of IL-4 gene expression by distal regulatory elements and GATA-3 at the chromatin level. Immunity $14(4): 447-459$.

Lentjes MH, Niessen HE, Akiyama Y, de Bruine AP, Melotte V, van Engeland M. 2016. The emerging role of GATA transcription factors in development and disease. Expert Rev Mol Med 18:e3.

Leonard MW, Lim KC, Engel JD. 1993. Expression of the chicken GATA factor family during early erythroid development and differentiation. Development 119(2):519-531.

Li Y, Esain V, Teng L, Xu J, Kwan W, Frost IM, Yzaguirre AD, Cai X, Cortes M, Maijenburg MW and others. 2014. Inflammatory signaling regulates embryonic hematopoietic stem and progenitor cell production. Genes Dev 28(23):2597-2612.

Li Y, Gao L, Hadland B, Tan K, Speck NA. 2017. CD27 marks murine embryonic hematopoietic stem cells and type II prehematopoietic stem cells. Blood 130(3):372-376. 
Li Z, Lan Y, He W, Chen D, Wang J, Zhou F, Wang Y, Sun H, Chen X, Xu C and others. 2012. Mouse embryonic head as a site for hematopoietic stem cell development. Cell Stem Cell 11(5):663-675.

Lichtinger M, Ingram R, Hannah R, Muller D, Clarke D, Assi SA, Lie ALM, Noailles L, Vijayabaskar MS, Wu M and others. 2012. RUNX1 reshapes the epigenetic landscape at the onset of haematopoiesis. EMBO J 31(22):4318-4333.

Lie ALM, Marinopoulou E, Li Y, Patel R, Stefanska M, Bonifer C, Miller C, Kouskoff V, Lacaud G. 2014. RUNX1 positively regulates a cell adhesion and migration program in murine hemogenic endothelium prior to blood emergence. Blood 124(11):e11-20.

Lim KC, Lakshmanan G, Crawford SE, Gu Y, Grosveld F, Engel JD. 2000. Gata3 loss leads to embryonic lethality due to noradrenaline deficiency of the sympathetic nervous system. Nat Genet 25(2):209-212.

Lin MI, Price EN, Boatman S, Hagedorn EJ, Trompouki E, Satishchandran S, Carspecken CW, Uong A, DiBiase A, Yang S and others. 2015. Angiopoietin-like proteins stimulate HSPC development through interaction with notch receptor signaling. Elife 4.

Lin Y, Yoder MC, Yoshimoto M. 2014. Lymphoid progenitor emergence in the murine embryo and yolk sac precedes stem cell detection. Stem Cells Dev 23(11):1168-1177.

Ling KW, Ottersbach K, van Hamburg JP, Oziemlak A, Tsai FY, Orkin SH, Ploemacher R, Hendriks RW, Dzierzak E. 2004. GATA-2 plays two functionally distinct roles during the ontogeny of hematopoietic stem cells. J Exp Med 200(7):871-882.

Lis R, Karrasch CC, Poulos MG, Kunar B, Redmond D, Duran JGB, Badwe 
CR, Schachterle W, Ginsberg M, Xiang J and others. 2017. Conversion of adult endothelium to immunocompetent haematopoietic stem cells. Nature 545(7655):439-445.

Long Y, Huang H. 2015. On signaling pathways: hematopoietic stem cell specification from hemogenic endothelium. Sci China Life Sci 58(12):1256-1261.

Ludin A, Itkin T, Gur-Cohen S, Mildner A, Shezen E, Golan K, Kollet 0, Kalinkovich A, Porat Z, D'Uva G and others. 2012. Monocytes-macrophages that express alpha-smooth muscle actin preserve primitive hematopoietic cells in the bone marrow. Nat Immunol 13(11):1072-1082.

MacLean AL, Lo Celso C, Stumpf MP. 2017. Concise Review: Stem Cell Population Biology: Insights from Hematopoiesis. Stem Cells 35(1):80-88.

Manaia A, Lemarchandel V, Klaine M, Max-Audit I, Romeo P, DieterlenLievre F, Godin I. 2000. Lmo2 and GATA-3 associated expression in intraembryonic hemogenic sites. Development 127(3):643-653.

Mansson R, Hultquist A, Luc S, Yang L, Anderson K, Kharazi S, Al-Hashmi S, Liuba K, Thoren L, Adolfsson J and others. 2007. Molecular evidence for hierarchical transcriptional lineage priming in fetal and adult stem cells and multipotent progenitors. Immunity 26(4):407-419.

Marcotte M, Sharma R, Bouchard M. 2014. Gene regulatory network of renal primordium development. Pediatr Nephrol 29(4):637-644.

Martin DI, Orkin SH. 1990. Transcriptional activation and DNA binding by the erythroid factor GF-1/NF-E1/Eryf 1. Genes Dev 4(11):1886-1898.

Mascarenhas MI, Bacon WA, Kapeni C, Fitch SR, Kimber G, Cheng SW, Li J, Green AR, Ottersbach K. 2016. Analysis of Jak2 signaling reveals resistance of mouse embryonic hematopoietic stem cells to myeloproliferative disease 
mutation. Blood 127(19):2298-2309.

Mascarenhas MI, Parker A, Dzierzak E, Ottersbach K. 2009. Identification of novel regulators of hematopoietic stem cell development through refinement of stem cell localization and expression profiling. Blood 114(21):4645-4653.

McGrath KE, Frame JM, Fegan KH, Bowen JR, Conway SJ, Catherman SC, Kingsley PD, Koniski AD, Palis J. 2015. Distinct Sources of Hematopoietic Progenitors Emerge before HSCs and Provide Functional Blood Cells in the Mammalian Embryo. Cell Rep 11(12):1892-1904.

McKinney-Freeman SL, Naveiras O, Yates F, Loewer S, Philitas M, Curran M, Park PJ, Daley GQ. 2009. Surface antigen phenotypes of hematopoietic stem cells from embryos and murine embryonic stem cells. Blood 114(2):268-278.

Medvinsky A, Dzierzak E. 1996. Definitive hematopoiesis is autonomously initiated by the AGM region. Cell 86(6):897-906.

Medvinsky A, Rybtsov S, Taoudi S. 2011. Embryonic origin of the adult hematopoietic system: advances and questions. Development 138(6):1017-1031.

Mehta A, Baltimore D. 2016. MicroRNAs as regulatory elements in immune system logic. Nat Rev Immunol 16(5):279-294.

Mendes SC, Robin C, Dzierzak E. 2005. Mesenchymal progenitor cells localize within hematopoietic sites throughout ontogeny. Development 132(5):1127-1136.

Mendez-Ferrer S, Battista M, Frenette PS. 2010. Cooperation of beta(2)and beta(3)-adrenergic receptors in hematopoietic progenitor cell mobilization. Ann N Y Acad Sci 1192:139-144.

Mendez-Ferrer S, Lucas D, Battista M, Frenette PS. 2008. Haematopoietic 
stem cell release is regulated by circadian oscillations. Nature 452(7186):442447.

Mendez-Ferrer S, Michurina TV, Ferraro F, Mazloom AR, Macarthur BD, Lira SA, Scadden DT, Ma'ayan A, Enikolopov GN, Frenette PS. 2010. Mesenchymal and haematopoietic stem cells form a unique bone marrow niche. Nature 466(7308):829-834.

Merrill SA, Brittingham ZD, Yuan X, Moliterno AR, Sperati CJ, Brodsky RA. 2017. Eculizumab cessation in atypical hemolytic uremic syndrome. Blood 130(3):368-372.

Mikkola HK, Fujiwara Y, Schlaeger TM, Traver D, Orkin SH. 2003. Expression of CD41 marks the initiation of definitive hematopoiesis in the mouse embryo. Blood 101(2):508-516.

Mikkola HK, Gekas C, Orkin SH, Dieterlen-Lievre F. 2005. Placenta as a site for hematopoietic stem cell development. Exp Hematol 33(9):1048-1054.

Minegishi N, Ohta J, Yamagiwa H, Suzuki N, Kawauchi S, Zhou Y, Takahashi S, Hayashi N, Engel JD, Yamamoto M. 1999. The mouse GATA-2 gene is expressed in the para-aortic splanchnopleura and aorta-gonads and mesonephros region. Blood 93(12):4196-4207.

Mirshekar-Syahkal B, Fitch SR, Ottersbach K. 2014. Concise review: From greenhouse to garden: the changing soil of the hematopoietic stem cell microenvironment during development. Stem Cells 32(7):1691-1700.

Moignard V, Gottgens B. 2014. Transcriptional mechanisms of cell fate decisions revealed by single cell expression profiling. Bioessays 36(4):419-426.

Moignard V, Woodhouse S, Fisher J, Gottgens B. 2013. Transcriptional hierarchies regulating early blood cell development. Blood Cells Mol Dis 
51(4):239-247.

Molkentin JD. 2000. The zinc finger-containing transcription factors GATA-4, -5, and -6. Ubiquitously expressed regulators of tissue-specific gene expression. J Biol Chem 275(50):38949-38952.

Moriguchi T, Takako N, Hamada M, Maeda A, Fujioka Y, Kuroha T, Huber RE, Hasegawa SL, Rao A, Yamamoto M and others. 2006. Gata3 participates in a complex transcriptional feedback network to regulate sympathoadrenal differentiation. Development 133(19):3871-3881.

Morrison SJ, Hemmati HD, Wandycz AM, Weissman IL. 1995. The purification and characterization of fetal liver hematopoietic stem cells. Proc Natl Acad Sci U S A 92(22):10302-10306.

Muller AM, Medvinsky A, Strouboulis J, Grosveld F, Dzierzak E. 1994. Development of hematopoietic stem cell activity in the mouse embryo. Immunity $1(4): 291-301$.

Neave B, Rodaway A, Wilson SW, Patient R, Holder N. 1995. Expression of zebrafish GATA 3 (gta3) during gastrulation and neurulation suggests a role in the specification of cell fate. Mech Dev 51(2-3):169-182.

Nerlov C, Querfurth E, Kulessa H, Graf T. 2000. GATA-1 interacts with the myeloid PU.1 transcription factor and represses PU.1-dependent transcription. Blood 95(8):2543-2551.

Nishiguchi Y, Ohmoto M, Koki J, Enomoto T, Kominami R, Matsumoto I, Hirota J. 2016. Bcl11b/Ctip2 is required for development of lingual papillae in mice. Dev Biol 416(1):98-110.

Nishikawa M, Tahara T, Hinohara A, Miyajima A, Nakahata T, Shimosaka A. 2001. Role of the microenvironment of the embryonic aorta-gonad- 
mesonephros region in hematopoiesis. Ann N Y Acad Sci 938:109-116.

North T, Gu TL, Stacy T, Wang Q, Howard L, Binder M, Marin-Padilla M, Speck NA. 1999. Cbfa2 is required for the formation of intra-aortic hematopoietic clusters. Development 126(11):2563-2575.

North TE, Goessling W, Peeters M, Li P, Ceol C, Lord AM, Weber GJ, Harris J, Cutting CC, Huang P and others. 2009. Hematopoietic stem cell development is dependent on blood flow. Cell 137(4):736-748.

Nostro MC, Cheng X, Keller GM, Gadue P. 2008. Wnt, activin, and BMP signaling regulate distinct stages in the developmental pathway from embryonic stem cells to blood. Cell Stem Cell 2(1):60-71.

Oh DS, Troester MA, Usary J, Hu Z, He X, Fan C, Wu J, Carey LA, Perou CM. 2006. Estrogen-Regulated Genes Predict Survival in Hormone Receptor-Positive Breast Cancers. Journal of Clinical Oncology 24(11):1656-1664.

Oh DS, Troester MA, Usary J, Hu Z, He X, Fan C, Wu J, Carey LA, Perou CM. 2006. Estrogen-regulated genes predict survival in hormone receptor-positive breast cancers. J Clin Oncol 24(11):1656-1664.

Omatsu Y, Sugiyama T, Kohara H, Kondoh G, Fujii N, Kohno K, Nagasawa T. 2010. The essential functions of adipo-osteogenic progenitors as the hematopoietic stem and progenitor cell niche. Immunity 33(3):387-399.

Orkin SH, Zon LI. 2008. Hematopoiesis: an evolving paradigm for stem cell biology. Cell 132(4):631-644.

Orquera DP, Nasif S, Low MJ, Rubinstein M, de Souza FS. 2016. Essential function of the transcription factor Rax in the early patterning of the mammalian hypothalamus. Dev Biol 416(1):212-224. 
Ottersbach K, Dzierzak E. 2005. The murine placenta contains hematopoietic stem cells within the vascular labyrinth region. Dev Cell 8(3):377387.

Ottersbach K, Dzierzak E. 2009. Analysis of the mouse placenta as a hematopoietic stem cell niche. Methods Mol Biol 538:335-346.

Ottersbach K, Dzierzak E. 2010. The placenta as a haematopoietic organ. Int J Dev Biol 54(6-7):1099-1106.

Ottersbach K, Smith A, Wood A, Gottgens B. 2010. Ontogeny of haematopoiesis: recent advances and open questions. Br J Haematol 148(3):343355.

Palis J. 2014. Primitive and definitive erythropoiesis in mammals. Front Physiol 5:3.

Palis J, Yoder MC. 2001. Yolk-sac hematopoiesis: the first blood cells of mouse and man. Exp Hematol 29(8):927-936.

Pandolfi PP, Roth ME, Karis A, Leonard MW, Dzierzak E, Grosveld FG, Engel JD, Lindenbaum MH. 1995. Targeted disruption of the GATA3 gene causes severe abnormalities in the nervous system and in fetal liver haematopoiesis. Nat Genet 11(1):40-44.

Pattyn A, Goridis C, Brunet JF. 2000. Specification of the central noradrenergic phenotype by the homeobox gene Phox2b. Mol Cell Neurosci 15(3):235-243.

Pauklin S, Vallier L. 2013. The cell-cycle state of stem cells determines cell fate propensity. Cell 155(1):135-147.

Peeters M, Ottersbach K, Bollerot K, Orelio C, de Bruijn M, Wijgerde M, 
Dzierzak E. 2009. Ventral embryonic tissues and Hedgehog proteins induce early AGM hematopoietic stem cell development. Development 136(15):2613-2621.

Pei XH, Bai F, Smith MD, Usary J, Fan C, Pai SY, Ho IC, Perou CM, Xiong Y. 2009. CDK inhibitor p18(INK4c) is a downstream target of GATA3 and restrains mammary luminal progenitor cell proliferation and tumorigenesis. Cancer Cell 15(5):389-401.

Perez-Andreu V, Roberts KG, Harvey RC, Yang W, Cheng C, Pei D, Xu H, Gastier-Foster J, E S, Lim JY and others. 2013. Inherited GATA3 variants are associated with Ph-like childhood acute lymphoblastic leukemia and risk of relapse. Nat Genet 45(12):1494-1498.

Pierce H, Zhang D, Magnon C, Lucas D, Christin JR, Huggins M, Schwartz GJ, Frenette PS. 2017. Cholinergic Signals from the CNS Regulate G-CSF-Mediated HSC Mobilization from Bone Marrow via a Glucocorticoid Signaling Relay. Cell Stem Cell 20(5):648-658 e644.

Rafii S, Kloss CC, Butler JM, Ginsberg M, Gars E, Lis R, Zhan Q, Josipovic P, Ding BS, Xiang J and others. 2013. Human ESC-derived hemogenic endothelial cells undergo distinct waves of endothelial to hematopoietic transition. Blood 121(5):770-780.

Ranganath S, Murphy KM. 2001. Structure and specificity of GATA proteins in Th2 development. Mol Cell Biol 21(8):2716-2725.

Reece DE. 2017. Breaking bad...proteins. Blood 129(15):2041-2042.

Rizzo F, Coffman JA, Arnone MI. 2016. An Elk transcription factor is required for Runx-dependent survival signaling in the sea urchin embryo. Dev Biol 416(1):173-186.

Robin C, Bollerot K, Mendes S, Haak E, Crisan M, Cerisoli F, Lauw I, 
Kaimakis P, Jorna R, Vermeulen M and others. 2009. Human placenta is a potent hematopoietic niche containing hematopoietic stem and progenitor cells throughout development. Cell Stem Cell 5(4):385-395.

Robin C, Ottersbach K, Durand C, Peeters M, Vanes L, Tybulewicz V, Dzierzak E. 2006. An unexpected role for IL-3 in the embryonic development of hematopoietic stem cells. Dev Cell 11(2):171-180.

Rybtsov S, Batsivari A, Bilotkach K, Paruzina D, Senserrich J, Nerushev O, Medvinsky A. 2014. Tracing the origin of the HSC hierarchy reveals an SCFdependent, IL-3-independent CD43(-) embryonic precursor. Stem Cell Reports $3(3): 489-501$.

Rybtsov S, Ivanovs A, Zhao S, Medvinsky A. 2016. Concealed expansion of immature precursors underpins acute burst of adult HSC activity in foetal liver. Development 143(8):1284-1289.

Rybtsov S, Sobiesiak M, Taoudi S, Souilhol C, Senserrich J, Liakhovitskaia A, Ivanovs A, Frampton J, Zhao S, Medvinsky A. 2011. Hierarchical organization and early hematopoietic specification of the developing HSC lineage in the AGM region. J Exp Med 208(6):1305-1315.

Rylski M, Welch JJ, Chen YY, Letting DL, Diehl JA, Chodosh LA, Blobel GA, Weiss MJ. 2003. GATA-1-mediated proliferation arrest during erythroid maturation. Mol Cell Biol 23(14):5031-5042.

Salter AB, Meadows SK, Muramoto GG, Himburg H, Doan P, Daher P, Russell L, Chen B, Chao NJ, Chute JP. 2009. Endothelial progenitor cell infusion induces hematopoietic stem cell reconstitution in vivo. Blood 113(9):2104-2107.

Sanchez MJ, Holmes A, Miles C, Dzierzak E. 1996. Characterization of the first definitive hematopoietic stem cells in the AGM and liver of the mouse embryo. Immunity 5(6):513-525. 
Sanda T, Lawton LN, Barrasa MI, Fan ZP, Kohlhammer H, Gutierrez A, Ma W, Tatarek J, Ahn Y, Kelliher MA and others. 2012. Core transcriptional regulatory circuit controlled by the TAL1 complex in human $\mathrm{T}$ cell acute lymphoblastic leukemia. Cancer Cell 22(2):209-221.

Sandler VM, Lis R, Liu Y, Kedem A, James D, Elemento O, Butler JM, Scandura JM, Rafii S. 2014. Reprogramming human endothelial cells to haematopoietic cells requires vascular induction. Nature 511(7509):312-318.

Scialdone A, Tanaka Y, Jawaid W, Moignard V, Wilson NK, Macaulay IC, Marioni JC, Gottgens B. 2016. Resolving early mesoderm diversification through single-cell expression profiling. Nature 535(7611):289-293.

Solaimani Kartalaei P, Yamada-Inagawa T, Vink CS, de Pater E, van der Linden R, Marks-Bluth J, van der Sloot A, van den Hout M, Yokomizo T, van Schaick-Solerno ML and others. 2015. Whole-transcriptome analysis of endothelial to hematopoietic stem cell transition reveals a requirement for Gpr56 in HSC generation. J Exp Med 212(1):93-106.

Souilhol C, Lendinez JG, Rybtsov S, Murphy F, Wilson H, Hills D, Batsivari A, Binagui-Casas A, McGarvey AC, MacDonald HR and others. 2016. Developing HSCs become Notch independent by the end of maturation in the AGM region. Blood 128(12):1567-1577.

Speck NA, Iruela-Arispe ML. 2009. Conditional Cre/LoxP strategies for the study of hematopoietic stem cell formation. Blood Cells Mol Dis 43(1):6-11.

Spiegel A, Shivtiel S, Kalinkovich A, Ludin A, Netzer N, Goichberg P, Azaria Y, Resnick I, Hardan I, Ben-Hur H and others. 2007. Catecholaminergic neurotransmitters regulate migration and repopulation of immature human CD34+ cells through Wnt signaling. Nat Immunol 8(10):1123-1131. 
Stemple DL, Anderson DJ. 1992. Isolation of a stem cell for neurons and glia from the mammalian neural crest. Cell 71(6):973-985.

Sugiyama T, Kohara H, Noda M, Nagasawa T. 2006. Maintenance of the hematopoietic stem cell pool by CXCL12-CXCR4 chemokine signaling in bone marrow stromal cell niches. Immunity 25(6):977-988.

Suzuki M, Kobayashi-Osaki M, Tsutsumi S, Pan X, Ohmori S, Takai J, Moriguchi T, Ohneda O, Ohneda K, Shimizu R and others. 2013. GATA factor switching from GATA2 to GATA1 contributes to erythroid differentiation. Genes Cells 18(11):921-933.

Swiers G, Baumann C, O'Rourke J, Giannoulatou E, Taylor S, Joshi A, Moignard V, Pina C, Bee T, Kokkaliaris KD and others. 2013. Early dynamic fate changes in haemogenic endothelium characterized at the single-cell level. Nat Commun 4:2924.

Swiers G, de Bruijn M, Speck NA. 2010. Hematopoietic stem cell emergence in the conceptus and the role of Runx1. Int J Dev Biol 54(6-7):11511163.

Swiers G, Rode C, Azzoni E, de Bruijn MF. 2013. A short history of hemogenic endothelium. Blood Cells Mol Dis 51(4):206-212.

Taichman RS, Reilly MJ, Emerson SG. 1996. Human osteoblasts support human hematopoietic progenitor cells in vitro bone marrow cultures. Blood 87(2):518-524.

Takahashi S, Shimizu R, Suwabe N, Kuroha T, Yoh K, Ohta J, Nishimura S, Lim KC, Engel JD, Yamamoto M. 2000. GATA factor transgenes under GATA-1 locus control rescue germline GATA-1 mutant deficiencies. Blood 96(3):910-916.

Takemoto N, Arai Ki, Miyatake S. 2002. Cutting Edge: The Differential 
Involvement of the N-Finger of GATA-3 in Chromatin Remodeling and Transactivation During Th2 Development. The Journal of Immunology 169(8):4103-4107.

Taoudi S, Gonneau C, Moore K, Sheridan JM, Blackburn CC, Taylor E, Medvinsky A. 2008. Extensive hematopoietic stem cell generation in the AGM region via maturation of VE-cadherin+CD45+ pre-definitive HSCs. Cell Stem Cell 3(1):99-108.

Taoudi S, Medvinsky A. 2007. Functional identification of the hematopoietic stem cell niche in the ventral domain of the embryonic dorsal aorta. Proc Natl Acad Sci U S A 104(22):9399-9403.

Taoudi S, Morrison AM, Inoue H, Gribi R, Ure J, Medvinsky A. 2005. Progressive divergence of definitive haematopoietic stem cells from the endothelial compartment does not depend on contact with the foetal liver. Development 132(18):4179-4191.

Tavian M, Coulombel L, Luton D, Clemente HS, Dieterlen-Lievre F, Peault B. 1996. Aorta-associated CD34+ hematopoietic cells in the early human embryo. Blood 87(1):67-72.

Tindemans I, Serafini N, Di Santo JP, Hendriks RW. 2014. GATA-3 function in innate and adaptive immunity. Immunity 41(2):191-206.

Tong Q, Dalgin G, Xu H, Ting CN, Leiden JM, Hotamisligil GS. 2000. Function of GATA transcription factors in preadipocyte-adipocyte transition. Science 290(5489):134-138.

Trainor CD, Omichinski JG, Vandergon TL, Gronenborn AM, Clore GM, Felsenfeld G. 1996. A palindromic regulatory site within vertebrate GATA-1 promoters requires both zinc fingers of the GATA-1 DNA-binding domain for high-affinity interaction. Mol Cell Biol 16(5):2238-2247. 
Tsai FY, Browne CP, Orkin SH. 1998. Knock-in mutation of transcription factor GATA-3 into the GATA-1 locus: partial rescue of GATA-1 loss of function in erythroid cells. Dev Biol 196(2):218-227.

Tsai FY, Keller G, Kuo FC, Weiss M, Chen J, Rosenblatt M, Alt FW, Orkin SH. 1994. An early haematopoietic defect in mice lacking the transcription factor GATA-2. Nature 371(6494):221-226.

Tsai FY, Orkin SH. 1997. Transcription factor GATA-2 is required for proliferation/survival of early hematopoietic cells and mast cell formation, but not for erythroid and myeloid terminal differentiation. Blood 89(10):3636-3643.

Tsarovina K, Pattyn A, Stubbusch J, Muller F, van der Wees J, Schneider C, Brunet JF, Rohrer H. 2004. Essential role of Gata transcription factors in sympathetic neuron development. Development 131(19):4775-4786.

Tsarovina K, Reiff T, Stubbusch J, Kurek D, Grosveld FG, Parlato R, Schutz G, Rohrer H. 2010. The Gata3 transcription factor is required for the survival of embryonic and adult sympathetic neurons. J Neurosci 30(32):10833-10843.

Turpen JB, Kelley CM, Mead PE, Zon LI. 1997. Bipotential primitivedefinitive hematopoietic progenitors in the vertebrate embryo. Immunity 7(3):325-334.

Usary J, Llaca V, Karaca G, Presswala S, Karaca M, He X, Langerod A, Karesen R, Oh DS, Dressler LG and others. 2004. Mutation of GATA3 in human breast tumors. Oncogene 23(46):7669-7678.

Van Handel B, Montel-Hagen A, Sasidharan R, Nakano H, Ferrari R, Boogerd CJ, Schredelseker J, Wang Y, Hunter S, Org T and others. 2012. Scl represses cardiomyogenesis in prospective hemogenic endothelium and endocardium. Cell 150(3):590-605. 
Vosshenrich CA, Garcia-Ojeda ME, Samson-Villeger SI, Pasqualetto V, Enault L, Richard-Le Goff O, Corcuff E, Guy-Grand D, Rocha B, Cumano A and others. 2006. A thymic pathway of mouse natural killer cell development characterized by expression of GATA-3 and CD127. Nat Immunol 7(11):12171224.

Wan YY. 2014. GATA3: a master of many trades in immune regulation. Trends Immunol 35(6):233-242.

Wang D, Li L, Lu J, Liu S, Shen J. 2016. Complementary expression of optomotor-blind and the Iroquois complex promotes fold formation to separate wing notum and hinge territories. Dev Biol 416(1):225-234.

Wang H, Liu C, Liu X, Wang M, Wu D, Gao J, Su P, Nakahata T, Zhou W, Xu Y and others. 2018. MEIS1 Regulates Hemogenic Endothelial Generation, Megakaryopoiesis, and Thrombopoiesis in Human Pluripotent Stem Cells by Targeting TAL1 and FLI1. Stem Cell Reports.

Wang Q, Stacy T, Binder M, Marin-Padilla M, Sharpe AH, Speck NA. 1996. Disruption of the Cbfa2 gene causes necrosis and hemorrhaging in the central nervous system and blocks definitive hematopoiesis. Proc Natl Acad Sci U S A 93(8):3444-3449.

Weisel KC, Moore MA. 2005. Genetic and functional characterization of isolated stromal cell lines from the aorta-gonado-mesonephros region. Ann N Y Acad Sci 1044:51-59.

Whyatt DJ, Karis A, Harkes IC, Verkerk A, Gillemans N, Elefanty AG, Vairo G, Ploemacher R, Grosveld F, Philipsen S. 1997. The level of the tissue-specific factor GATA-1 affects the cell-cycle machinery. Genes Funct 1(1):11-24.

Wilson A, Laurenti E, Oser G, van der Wath RC, Blanco-Bose W, Jaworski 
M, Offner S, Dunant CF, Eshkind L, Bockamp E and others. 2008. Hematopoietic stem cells reversibly switch from dormancy to self-renewal during homeostasis and repair. Cell 135(6):1118-1129.

Yamashita M, Ukai-Tadenuma M, Miyamoto T, Sugaya K, Hosokawa H, Hasegawa A, Kimura M, Taniguchi M, DeGregori J, Nakayama T. 2004. Essential role of GATA3 for the maintenance of type 2 helper T (Th2) cytokine production and chromatin remodeling at the Th2 cytokine gene loci. J Biol Chem 279(26):26983-26990.

Yoder MC, Hiatt K, Dutt P, Mukherjee P, Bodine DM, Orlic D. 1997. Characterization of definitive lymphohematopoietic stem cells in the day 9 murine yolk sac. Immunity 7(3):335-344.

Zhang C, Lv J, He Q, Wang S, Gao Y, Meng A, Yang X, Liu F. 2014. Inhibition of endothelial ERK signalling by Smad1/5 is essential for haematopoietic stem cell emergence. Nat Commun 5:3431.

Zhang J, Ding L, Holmfeldt L, Wu G, Heatley SL, Payne-Turner D, Easton J, Chen X, Wang J, Rusch M and others. 2012. The genetic basis of early T-cell precursor acute lymphoblastic leukaemia. Nature 481(7380):157-163.

Zhao GY, Li ZY, Zou HL, Hu ZL, Song NN, Zheng MH, Su CJ, Ding YQ. 2008. Expression of the transcription factor GATA3 in the postnatal mouse central nervous system. Neurosci Res 61(4):420-428.

Zhong JF, Zhao Y, Sutton S, Su A, Zhan Y, Zhu L, Yan C, Gallaher T, Johnston PB, Anderson WF and others. 2005. Gene expression profile of murine long-term reconstituting vs. short-term reconstituting hematopoietic stem cells. Proc Natl Acad Sci U S A 102(7):2448-2453.

Zhou F, Li X, Wang W, Zhu P, Zhou J, He W, Ding M, Xiong F, Zheng X, Li Z and others. 2016. Tracing haematopoietic stem cell formation at single-cell 
resolution. Nature 533(7604):487-492.

Zhou M, Ouyang W. 2003. The function role of GATA-3 in Th1 and Th2 differentiation. Immunol Res 28(1):25-37.

Zovein AC, Hofmann JJ, Lynch M, French WJ, Turlo KA, Yang Y, Becker MS, Zanetta L, Dejana E, Gasson JC and others. 2008. Fate tracing reveals the endothelial origin of hematopoietic stem cells. Cell Stem Cell 3(6):625-636. 